\title{
Simplified interval-valued Pythagorean fuzzy graphs with application
}

\author{
Muhammad Akram ${ }^{1}$ (I) $\cdot$ Sumera $\mathrm{Naz}^{1} \cdot$ Bijan Davvaz $^{1}$ \\ Received: 8 October 2018 / Accepted: 12 April 2019 / Published online: 3 May 2019 \\ (c) The Author(s) 2019
}

\begin{abstract}
Interval-valued Pythagorean fuzzy set (IVPFS) as a generalization of Pythagorean fuzzy set (PFS) increases its elasticity drastically. However, the expressions and calculations of IVPFS are slightly complicated. To overcome this drawback, in this research study, we greatly simplify the expressions of IVPFS by introducing a new concept of simplified interval-valued Pythagorean fuzzy set (SIVPFS), constituted by two Pythagorean fuzzy numbers (PFNs) with the relationships of intersection and union simultaneously. We develop systematic aggregation operators to aggregate simplified interval-valued Pythagorean fuzzy information. Meanwhile, we propose a new generalization of fuzzy graph, called simplified interval-valued Pythagorean fuzzy graph (SIVPFG), to describe uncertain information in graph theory. We develop a series of operations on two SIVPFGs and investigate their desirable properties. Finally, we develop a SIVPFG-based multi-agent decision-making approach to solve a common kind of situation where the graphic structure of agents is obscure. A numerical example is provided to illustrate the proposed approach as well as the applicability of SIVPFS and SIVPFG in decision making.
\end{abstract}

Keywords Simplified interval-valued Pythagorean fuzzy set · Simplified interval-valued Pythagorean fuzzy graph · Degree of a vertex $\cdot$ Total degree of a vertex $\cdot$ Multi-agent decision making

\section{Introduction}

As an effective framework, multi-criteria decision making (MCDM) has consistently been used to choose the optimal alternative(s) from a given finite set of alternatives with respect to a collection of criteria. Nowadays, it has been applied to several scientific fields, such as environmental impact assessment, manufacturing systems and location selection. In various real-world problems, due to some influencing factors, such as limited budgets, tight deadlines and limited domain knowledge of the expert, it is quite difficult for experts to give their assessments on performance ratings and attribute weights with precise values. However, the fuzzy set [37] is suitable to describe the uncertainties when one evaluates decision options for the MCDM problems. Consequently, many MCDM methods have been suggested under fuzzy, interval-valued fuzzy, intuitionistic fuzzy and hesitant fuzzy environment.

Yager's Pythagorean fuzzy set [32-34], a useful generalization of intuitionistic fuzzy set [7], is a new tool used to model imprecise and obscure information in multi-attribute

Muhammad Akram

m.akram@pucit.edu.pk

1 University of the Punjab, Lahore, Pakistan decision-making problems. The prominent characteristic of the Pythagorean fuzzy model is to relax the condition that the sum of its membership degree and non-membership degree is not greater than one with the square sum of its membership degree and non-membership degree not being greater than one. Obviously, PFSs have higher potentiality than IFSs to model the obscurity and to manage the complex impreciseness and uncertainty in the practical multiattribute decision-making problems. Garg [12-14] developed novel decision-making approaches under the Pythagorean fuzzy environment. Akram et al. [1] extended the TOPSIS method to solve multi-criteria group decision-making problems equipped with Pythagorean fuzzy data. Recently, Peng and Yang [25] initiated the concept of IVPFSs as a generalization of PFSs in the spirit of interval-valued fuzzy sets [36]. Its characteristic is that an interval-valued membership degree and an interval-valued non-membership degree are assigned to each element in the set. When the values of the membership function and the non-membership function in a PFS are difficult to be expressed as exact real numbers in many real-world problems, IVPFS can be used to characterize the uncertain information more sufficiently and accurately. IVPFS has been widely applied in many fields of modern society, such as decision making $[9,10,28]$. Further, a 
novel accuracy function [15], improved score function [16], exponential operational laws [17] and Maclaurin symmetric mean operators [30] have been defined in interval-valued Pythagorean fuzzy environment and have been applied to solve the multi-attribute decision-making problems. More recently, Maclaurin symmetric mean operators have been developed with the hesitant Pythagorean fuzzy information.

It is quite well known that a graph is simply a model of relations and is a suitable way of depicting information comprising relationship between objects (vertices). For instance, in the Internet, a router can be represented as a vertex and an edge connects two routers with optical fiber. With the development of system complexity, a variety of uncertain information is frequently encountered in networks. To deal with this uncertain or vague information, Rosenfeld [29] proposed the concept of fuzzy graphs. Fuzzy graph operations were defined by Mordeson and Peng [20]. Yu and Xu [35] proposed multi-agent decision-making model based on graphs, to solve a kind of MCDM problems along with the interrelated criteria. With the more and more obscure information in the networks, different extensions of fuzzy graph [19,21-23] have been introduced by several researchers. Intuitionistic fuzzy graph developed by Akram and Davvaz [5] is a generalization of fuzzy graph, which has been found to be excessively useful in dealing with vagueness. Naz et al. [24] recently introduced the new concept of Pythagorean fuzzy graphs (PFGs), as an extended version of intuitionistic fuzzy graphs [5]. Akram et al. [2-4] put forward certain concepts of graphs in Pythagorean fuzzy circumstances. The PFG is more flexible and more practical than fuzzy graphs and IFGs. However, due to insufficiency in available information from some practical decision-making problems with the interrelated criteria, it may be tough for the experts to exactly quantify their judgment with a classical number, but can represent them by an interval number in $[0,1]$. Therefore, it is very significant to introduce the concept of interval-valued Pythagorean fuzzy graphs (IVPFGs), which permits the membership degree and the non-membership degree of an element in the given set of vertices and edges to have an interval value.

IVPFSs are very practical in expressing the variations of the membership and the non-membership function under a fuzzy environment. But unfortunately, its traditional depictions seem too complicated. To overcome this difficulty, in this research study, we greatly simplify the expressions of IVPFS by introducing a new concept of simplified intervalvalued Pythagorean fuzzy set (SIVPFS) and the simplified interval-valued Pythagorean fuzzy number (SIVPFN), characterized by two PFNs, as a basic element of a SIVPFS. We develop systematic operations and aggregation operators to aggregate simplified interval-valued Pythagorean fuzzy information. By proposing a simplified way, the calculations of the operations and aggregation operators of interval-valued Pythagorean fuzzy information will be sim- pler and convenient for actual applications. Moreover, within the framework of proposed SIVPFS theory, we introduce the novel concept of simplified interval-valued Pythagorean fuzzy graphs (SIVPFGs). We develop a series of operations on two SIVPFGs, and investigate their desirable properties in terms of the degree and the total degree of a vertex. Finally, we develop a SIVPFG-based MCDM approach to solve a general kind of situation where the agents' graphic structure is obscure.

The paper is structured as follows: "Simplified intervalvalued Pythagorean fuzzy set" section puts forward a new concept of SIVPFS along with a series of its operational laws and aggregation operators. In "Simplified interval-valued Pythagorean fuzzy graphs" section, we propose a new generalization of Pythagorean fuzzy graphs, called SIVPFG and investigate its properties in detail. "Decision-making approach based on the proposed SIVPFGs" section is devoted to the application of SIVPFSs and SIVPFGs in MCDM, and finally we draw conclusions and elaborate on future work in "Conclusions" section.

\section{Simplified interval-valued Pythagorean fuzzy set}

Peng and Yang [25] introduced the concept of IVPFS which is a generalization of PFS and interval-valued intuitionistic fuzzy set [8].

Definition 1 [25] Let $D[0,1]$ represent the set of all closed subintervals of $[0,1]$, and $Z$ be a universe of discourse. An IVPFS $\tilde{\mathcal{P}}$ in $Z$ is an object of the form

$\tilde{\mathcal{P}}=\left\{\left\langle z, \tilde{\mu}_{\tilde{\mathcal{P}}}(z), \tilde{v}_{\tilde{\mathcal{P}}}(z)\right\rangle \mid z \in Z\right\}$,

where the functions $\tilde{\mu}_{\tilde{\mathcal{P}}}: Z \rightarrow D[0,1]\left(z \in Z \rightarrow \tilde{\mu}_{\tilde{\mathcal{P}}}(z) \subseteq\right.$ $[0,1])$ and $\tilde{v}_{\tilde{\mathcal{P}}}: Z \rightarrow D[0,1]\left(z \in Z \rightarrow \tilde{v}_{\tilde{\mathcal{P}}}(z) \subseteq[0,1]\right)$ denote the membership degree and non-membership degree of the element $z \in Z$ to the set $\tilde{\mathcal{P}}$, respectively, and for each $z \in Z, \tilde{\mu}_{\tilde{\mathcal{P}}}(z)$ and $\tilde{v}_{\tilde{\mathcal{P}}}(z)$ are closed intervals and their lower and upper bounds are denoted by $\tilde{\mu}_{\tilde{\mathcal{P}}}^{L}(z), \tilde{\mu}_{\tilde{\mathcal{P}}}^{U}(z)$ and $\tilde{v}_{\tilde{\mathcal{P}}}^{L}(z)$, $\tilde{v}_{\tilde{\mathcal{P}}}^{U}(z)$, respectively, such that $\left(\tilde{\mu}_{\tilde{\mathcal{P}}}^{U}(z)\right)^{2}+\left(\tilde{v}_{\tilde{\mathcal{P}}}^{U}(z)\right)^{2} \leq 1$.

For every $z \in Z, \tilde{\pi}_{\tilde{\mathcal{P}}}(z)=\left[\tilde{\pi}_{\tilde{\mathcal{P}}}^{L}(z), \tilde{\pi}_{\tilde{\mathcal{P}}}^{U}(z)\right]=$ $\left[\sqrt{1-\left(\tilde{\mu}_{\tilde{\mathcal{P}}}^{U}(z)\right)^{2}+\left(\tilde{v}_{\tilde{\mathcal{P}}}^{U}(z)\right)^{2}}, \sqrt{1-\left(\tilde{\mu}_{\tilde{\mathcal{P}}}^{L}(z)\right)^{2}+\left(\tilde{v}_{\tilde{\mathcal{P}}}^{L}(z)\right)^{2}}\right]$ is called an interval-valued Pythagorean fuzzy index of $z$ to $\tilde{\mathcal{P}}$.

The main difference between interval-valued intuitionistic fuzzy number (IVIFN) and interval-valued Pythagorean fuzzy number (IVPFN) is their different constraint conditions, as shown in Fig. 1. An IVIFS is a region, constituted by two closed intervals $\left[\tilde{\mu}_{\tilde{A}}^{L}(z), \tilde{\mu}_{\tilde{A}}^{U}(z)\right]$ and $\left[\tilde{v}_{\tilde{A}}^{L}(z), \tilde{v}_{\tilde{A}}^{U}(z)\right]$, which utilizes the length and width of the rectangle, as shown 


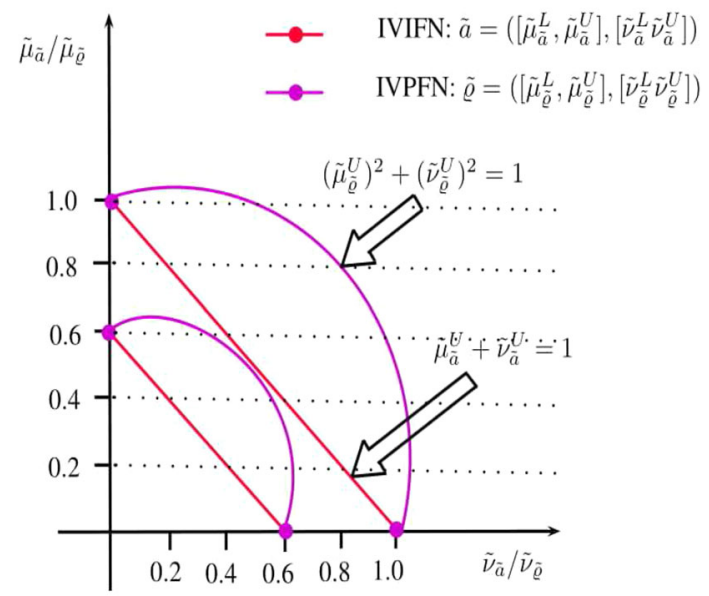

Fig. 1 Comparison of spaces of the IVIFNs and the IVPFNs

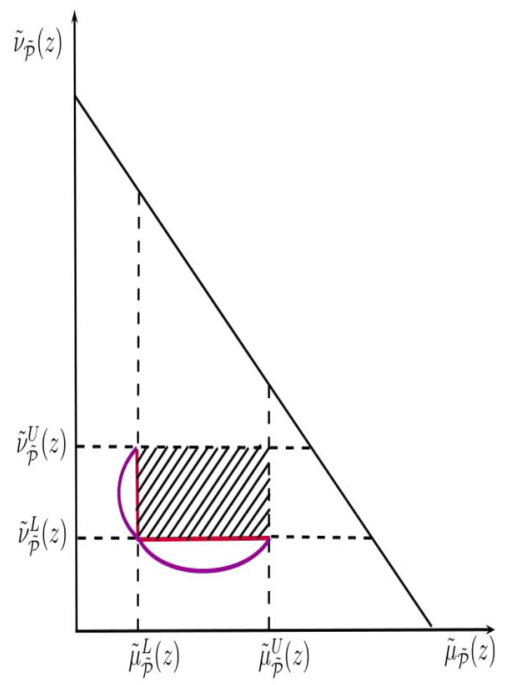

Fig. 2 The interval-valued Pythagorean fuzzy set

in Fig. 2. The diagonal line of a rectangle manifests the character of the rectangle in a coordinate axis plane and is more convenient and simple. Based on Fig. 2, choose the worst and the best values to express IVIFS and describe these two values as two ordered IFNs $\alpha=\left(\mu_{\alpha}, v_{\alpha}\right)$ and $\beta=\left(\mu_{\beta}, v_{\beta}\right)$, as shown in Fig. 3 [27].

While, an IVPFS is a region constituted by two closed intervals $\left[\tilde{\mu}_{\tilde{\mathcal{P}}}^{L}(z), \tilde{\mu}_{\tilde{\mathcal{P}}}^{U}(z)\right]$ and $\left[\tilde{v}_{\tilde{\mathcal{P}}}^{L}(z), \tilde{v}_{\tilde{\mathcal{P}}}^{U}(z)\right]$, which utilizes the curves along the length and width of the rectangle to express the IVPFS. Similar to IVIFS, we can choose the worst and the best values to express IVPFS and can describe these two values as two ordered PFNs $\zeta=\left(\mu_{\zeta}, v_{\zeta}\right)$ and $\eta=$ $\left(\mu_{\eta}, v_{\eta}\right)$, as shown in Fig 3.

Based on the above analysis, the concept of SIVPFS can be defined as:

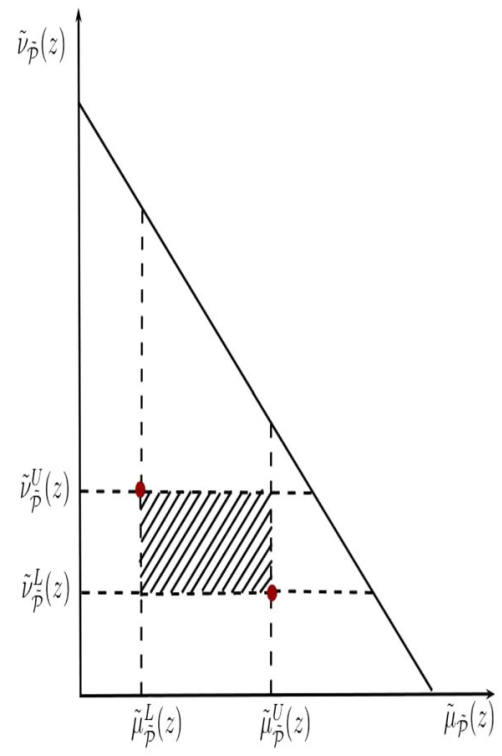

Fig. 3 The simplified interval-valued Pythagorean fuzzy set

Definition 2 Let $Z$ be a fixed set. Then

$\tilde{\mathcal{P}}=\{\langle z, \zeta, \eta\rangle \mid z \in Z\}$

is called a SIVPFS, where $\zeta=\left(\tilde{\mu}_{\tilde{\mathcal{P}}}^{L}(z), \tilde{v}_{\tilde{\mathcal{P}}}^{U}(z)\right)$ and $\eta=$ $\left(\tilde{\mu}_{\tilde{\mathcal{P}}}^{U}(z), \tilde{v}_{\tilde{\mathcal{P}}}^{L}(z)\right)$ are two Pythagorean fuzzy numbers, satisfying $\zeta \cup \eta=\eta$ or $\zeta \cap \eta=\zeta$.

Theorem 1 Let $\tilde{\mathcal{P}}=\{\langle z, \zeta, \eta\rangle \mid z \in Z\}, \tilde{\mathcal{P}}_{1}=\left\{\left\langle z, \zeta_{1}, \eta_{1}\right\rangle\right.$ $\mid z \in Z\}$ and $\tilde{\mathcal{P}}_{2}=\left\{\left\langle z, \zeta_{2}, \eta_{2}\right\rangle \mid z \in Z\right\}$ be three SIVPFSs, where $\zeta=\left(\tilde{\mu}_{\tilde{\mathcal{P}}}^{L}(z), \tilde{v}_{\tilde{\mathcal{P}}}^{U}(z)\right) \eta=\left(\tilde{\mu}_{\tilde{\mathcal{P}}}^{U}(z), \tilde{v}_{\tilde{\mathcal{P}}}^{L}(z)\right)$, $\zeta_{1}=\left(\tilde{\mu}_{\tilde{\mathcal{P}}_{1}}^{L}(z), \tilde{v}_{\tilde{\mathcal{P}}_{1}}^{U}(z)\right), \eta_{1}=\left(\tilde{\mu}_{\tilde{\mathcal{P}}_{1}}^{U}(z), \tilde{v}_{\tilde{\mathcal{P}}_{1}}^{L}(z)\right), \zeta_{2}=$ $\left(\tilde{\mu}_{\tilde{\mathcal{P}}_{2}}^{L}(z), \tilde{v}_{\tilde{\mathcal{P}}_{2}}^{U}(z)\right), \eta_{2}=\left(\tilde{\mu}_{\tilde{\mathcal{P}}_{2}}^{U}(z), \tilde{v}_{\tilde{\mathcal{P}}_{2}}^{L}(z)\right)$. Then

1. $\tilde{\mathcal{P}}_{1} \prec \tilde{\mathcal{P}}_{2}$ if and only if $\zeta_{1} \prec \zeta_{2}, \eta_{1} \prec \eta_{2}$;

2. $\overline{\tilde{\mathcal{P}}}=\{\langle z, \bar{\eta}, \bar{\zeta}\rangle \mid z \in Z\}$;

3. $\tilde{\mathcal{P}}_{1} \cap \tilde{\mathcal{P}}_{2}=\left\{\left\langle z, \zeta_{1} \cap \zeta_{2}, \eta_{1} \cap \eta_{2}\right\rangle \mid z \in Z\right\}$;

4. $\tilde{\mathcal{P}}_{1} \cup \tilde{\mathcal{P}}_{2}=\left\{\left\langle z, \zeta_{1} \cup \zeta_{2}, \eta_{1} \cup \eta_{2}\right\rangle \mid z \in Z\right\}$;

5. $\tilde{\mathcal{P}}_{1}+\tilde{\mathcal{P}}_{2}=\left\{\left\langle z, \zeta_{1} \oplus \zeta_{2}, \eta_{1} \oplus \eta_{2}\right\rangle \mid z \in Z\right\}$;

6. $\tilde{\mathcal{P}}_{1} . \tilde{\mathcal{P}}_{2}=\left\{\left\langle z, \zeta_{1} \otimes \zeta_{2}, \eta_{1} \otimes \eta_{2}\right\rangle \mid z \in Z\right\}$;

7. $\lambda \tilde{\mathcal{P}}=\{\langle z, \lambda \zeta, \lambda \eta\rangle \mid z \in Z\}, \quad \lambda>0$;

8. $\tilde{\mathcal{P}}^{\lambda}=\left\{\left\langle z, \zeta^{\lambda}, \eta^{\lambda}\right\rangle \mid z \in Z\right\}, \quad \lambda>0$.

Proof The proof is straightforward.

For convenience, we call $\tilde{\varrho}=\langle\zeta, \eta\rangle$ a simplified intervalvalued Pythagorean fuzzy number (SIVPFN), constituted by two PFNs $\zeta=\left(\tilde{\mu}_{\tilde{\varrho}}^{L}, \tilde{v}_{\tilde{\varrho}}^{U}\right)$ and $\eta=\left(\tilde{\mu}_{\tilde{\varrho}}^{U}, \tilde{v}_{\tilde{\varrho}}^{L}\right)$.

Definition 3 Let $\tilde{\varrho}_{j}=\left\langle\left(\tilde{\mu}_{j}^{L}, \tilde{v}_{j}^{U}\right),\left(\tilde{\mu}_{j}^{U}, \tilde{v}_{j}^{L}\right)\right\rangle(j=1,2$, $\ldots, k)$ be a collection of SIVPFNs, the simplified intervalvalued Pythagorean fuzzy weighted averaging (SIVPFWA) operator is a mapping SIVPFWA: $(\tilde{\mathcal{P}})^{k} \rightarrow \tilde{\mathcal{P}}$, where 
$\operatorname{SIVPFWA}\left(\tilde{\varrho}_{1}, \tilde{\varrho}_{2}, \ldots, \tilde{\varrho}_{k}\right)=\sum_{j=1}^{k} w_{j} \tilde{\varrho}_{j}$

$w=\left(w_{1}, w_{2}, \ldots, w_{k}\right)^{T}$ is the weight vector of $\tilde{\varrho}_{j}(j=$ $1,2, \ldots, k)$, satisfying $w_{j} \in[0,1](j=1,2, \ldots, k)$ and $\sum_{j=1}^{k} w_{j}=1$.

With the operations of SIVPFNs, by induction on $k$, we get SIVPFWA operator as:

$\operatorname{SIVPFWA}\left(\tilde{\varrho}_{1}, \tilde{\varrho}_{2}, \ldots, \tilde{\varrho}_{k}\right)$

$$
\begin{aligned}
= & \left\langle\left(\sqrt{1-\prod_{j=1}^{k}\left(1-\left(\tilde{\mu}_{j}^{L}\right)^{2}\right)^{w_{j}}}, \prod_{j=1}^{k}\left(\tilde{v}_{j}^{U}\right)^{w_{j}}\right),\right. \\
& \left.\times\left(\sqrt{1-\prod_{j=1}^{k}\left(1-\left(\tilde{\mu}_{j}^{U}\right)^{2}\right)^{w_{j}}}, \prod_{j=1}^{k}\left(\tilde{v}_{j}^{L}\right)^{w_{j}}\right)\right\rangle .
\end{aligned}
$$

Definition 4 Let $\tilde{\varrho}_{j}=\left\langle\left(\tilde{\mu}_{j}^{L}, \tilde{v}_{j}^{U}\right),\left(\tilde{\mu}_{j}^{U}, \tilde{v}_{j}^{L}\right)\right\rangle(j=1,2$, $\ldots, k)$ be a collection of SIVPFNs, the simplified intervalvalued Pythagorean fuzzy weighted geometric (SIVPFWG) operator is a mapping SIVPFWG: $(\tilde{\mathcal{P}})^{k} \rightarrow \tilde{\mathcal{P}}$, where

$\operatorname{SIVPFWG}\left(\tilde{\varrho}_{1}, \tilde{\varrho}_{2}, \ldots, \tilde{\varrho}_{k}\right)=\prod_{j=1}^{k}\left(\tilde{\varrho}_{j}\right)^{w_{j}}$

$w=\left(w_{1}, w_{2}, \ldots, w_{k}\right)^{T}$ is the weight vector of $\tilde{\varrho}_{j}(j=$ $1,2, \ldots, k)$, satisfying $w_{j} \in[0,1](j=1,2, \ldots, k)$ and $\sum_{j=1}^{k} w_{j}=1$.

With the operations of SIVPFNs, by induction on $k$, we get SIVPFWG operator as:

$\operatorname{SIVPFWA}\left(\tilde{\varrho}_{1}, \tilde{\varrho}_{2}, \ldots, \tilde{\varrho}_{k}\right)$

$$
\begin{aligned}
= & \left\langle\left(\prod_{j=1}^{k}\left(\tilde{v}_{j}^{U}\right)^{w_{j}}, \sqrt{1-\prod_{j=1}^{k}\left(1-\left(\tilde{\mu}_{j}^{L}\right)^{2}\right)^{w_{j}}}\right),\right. \\
& \left.\times\left(\prod_{j=1}^{k}\left(\tilde{v}_{j}^{L}\right)^{w_{j}}, \sqrt{1-\prod_{j=1}^{k}\left(1-\left(\tilde{\mu}_{j}^{U}\right)^{2}\right)^{w_{j}}}\right)\right\rangle .
\end{aligned}
$$

Especially, if $w=(1 / n, 1 / n, \ldots, 1 / n)^{T}$, then the SIVPFWA and SIVPFWG operators reduce to the simplified interval-valued Pythagorean fuzzy averaging (SIVPFA) and the simplified interval-valued Pythagorean fuzzy geometric (SIVPFG) operators, respectively.

Definition 5 Let $\tilde{\varrho}_{j}=\left\langle\left(\tilde{\mu}_{j}^{L}, \tilde{v}_{j}^{U}\right),\left(\tilde{\mu}_{j}^{U}, \tilde{v}_{j}^{L}\right)\right\rangle(j=1,2$, $\ldots, k)$ be a collection of SIVPFNs and $w=\left(w_{1}, w_{2}, \ldots\right.$, $\left.w_{k}\right)^{T}$ be the weight vector of $\tilde{\varrho}_{j}(j=1,2, \ldots, k)$, satisfying $w_{j} \in[0,1](j=1,2, \ldots, k)$ and $\sum_{j=1}^{k} w_{j}=1$, the simplified interval-valued Pythagorean fuzzy ordered weighted averaging (SIVPFOWG) operator is a mapping SIVPFOWG: $(\tilde{\mathcal{P}})^{k} \rightarrow \tilde{\mathcal{P}}$, where

$$
\operatorname{SIVPFOWG}\left(\tilde{\varrho}_{1}, \tilde{\varrho}_{2}, \ldots, \tilde{\varrho}_{k}\right)=\sum_{j=1}^{k} w_{j} \tilde{\varrho}_{\sigma(j)}
$$

With the operations of SIVPFNs, by induction on $k$, we get SIVPFOWA operator as:

$\operatorname{SIVPFWA}\left(\tilde{\varrho}_{1}, \tilde{\varrho}_{2}, \ldots, \tilde{\varrho}_{k}\right)$

$$
\begin{aligned}
= & \left\langle\left(\sqrt{1-\prod_{j=1}^{k}\left(1-\left(\tilde{\mu}_{\sigma(j)}^{L}\right)^{2}\right)^{w_{j}}}, \prod_{j=1}^{k}\left(\tilde{v}_{\sigma(j)}^{U}\right)^{w_{j}}\right),\right. \\
& \left.\times\left(\sqrt{1-\prod_{j=1}^{k}\left(1-\left(\tilde{\mu}_{\sigma(j)}^{U}\right)^{2}\right)^{w_{j}}}, \prod_{j=1}^{k}\left(\tilde{v}_{\sigma(j)}^{L}\right)^{w_{j}}\right)\right\rangle .
\end{aligned}
$$

Definition 6 Let $\tilde{\varrho}_{j}=\left\langle\left(\tilde{\mu}_{j}^{L}, \tilde{v}_{j}^{U}\right),\left(\tilde{\mu}_{j}^{U}, \tilde{v}_{j}^{L}\right)\right\rangle(j=1,2$, $\ldots, k)$ be a collection of SIVPFNs and $w=\left(w_{1}, w_{2}, \ldots\right.$, $\left.w_{k}\right)^{T}$ be the weight vector of $\tilde{\varrho}_{j}(j=1,2, \ldots, k)$, satisfying $w_{j} \in[0,1](j=1,2, \ldots, k)$ and $\sum_{j=1}^{k} w_{j}=1$, then the simplified interval-valued Pythagorean fuzzy ordered weighted geometric (SIVPFOWG) operator is a mapping SIVPFOWG: $(\tilde{P})^{k} \rightarrow \tilde{P}$, where

$\operatorname{SIVPFOWG}\left(\tilde{\varrho}_{1}, \tilde{\varrho}_{2}, \ldots, \tilde{\varrho}_{k}\right)=\sum_{j=1}^{k}\left(\tilde{\varrho}_{\sigma(j)}\right)^{w_{j}}$.

With the operations of SIVPFNs, by induction on $k$, we get SIVPFOWA operator as:

$$
\begin{aligned}
& \operatorname{SIVPFWA}\left(\tilde{\varrho}_{1}, \tilde{\varrho}_{2}, \ldots, \tilde{\varrho}_{k}\right) \\
& =\left\langle\left(\prod_{j=1}^{k}\left(\tilde{v}_{\sigma(j)}^{U}\right)^{w_{j}}, \sqrt{1-\prod_{j=1}^{k}\left(1-\left(\tilde{\mu}_{\sigma(j)}^{L}\right)^{2}\right)^{w_{j}}}\right),\right. \\
& \left.\quad \times\left(\prod_{j=1}^{k}\left(\tilde{v}_{\sigma(j)}^{L}\right)^{w_{j}}, \sqrt{1-\prod_{j=1}^{k}\left(1-\left(\tilde{\mu}_{\sigma(j)}^{U}\right)^{2}\right)^{w_{j}}}\right)\right\rangle
\end{aligned}
$$

Particularly, if $w=(1 / n, 1 / n, \ldots, 1 / n)^{T}$, then the SIVPFOWA and SIVPFOWG operators reduce to the SIVPFWA and SIVPFWG operators, respectively.

The SIVPFWA and SIVPFWG operators only weigh the SIVPFNs, and the SIVPFOWA and SIVPFOWG operators only weigh the ordered position of the SIVPFNs. In real applications, if we consider the weights of the SIVPFNs, we choose the SIVPFWA or SIVPFWG operator, and if we consider the ordered position of the SIVPFNs, we choose the SIVPFOWA or SIVPFOWG operator. 
Definition 7 Let $\tilde{\varrho}_{j}=\left\langle\left(\tilde{\mu}_{j}^{L}, \tilde{v}_{j}^{U}\right),\left(\tilde{\mu}_{j}^{U}, \tilde{v}_{j}^{L}\right)\right\rangle(j=1,2$, $\ldots, k)$ be a collection of SIVPFNs, the simplified intervalvalued Pythagorean fuzzy weighted combination (SIVPFWC) operator is a mapping SIVPFWC: $(\tilde{\mathcal{P}})^{k} \rightarrow \tilde{\mathcal{P}}$, where

$\operatorname{SIVPFWC}\left(\tilde{\varrho}_{1}, \tilde{\varrho}_{2}, \ldots, \tilde{\varrho}_{k}\right)=\vee_{j=1}^{k}\left(w_{j} \wedge \tilde{\varrho}_{j}\right)$

$w=\left(w_{1}, w_{2}, \ldots, w_{k}\right)^{T}$ is the weight vector of $\tilde{\varrho}_{j}(j=$ $1,2, \ldots, k)$, satisfying $w_{j} \in[0,1](j=1,2, \ldots, k)$ and $\sum_{j=1}^{k} w_{j}=1$.

\section{Simplified interval-valued Pythagorean fuzzy graphs}

In this section, first the concept of IVPFGs is introduced. Then the novel concept of SIVPFGs is put forward. A series of operational laws of SIVPFGs is given and related properties are investigated.

Definition 8 An IVPFG on a non-empty set $Z$ is a pair $\tilde{\mathcal{G}}=$ $(\tilde{P}, \tilde{Q})$, where $\tilde{P}$ is an IVPFS on $Z$ and $\tilde{Q}$ is an intervalvalued Pythagorean fuzzy relation on $Z$ such that

$\tilde{\mu}_{\tilde{Q}}^{L}(y z) \leq \min \left\{\tilde{\mu}_{\tilde{P}}^{L}(y), \tilde{\mu}_{\tilde{P}}^{L}(z)\right\}$,

$\tilde{\mu}_{\tilde{Q}}^{U}(y z) \leq \min \left\{\tilde{\mu}_{\tilde{P}}^{U}(y), \tilde{\mu}_{\tilde{P}}^{U}(z)\right\}$,

$\tilde{v}_{\tilde{Q}}^{L}(y z) \geq \max \left\{\tilde{v}_{\tilde{P}}^{L}(y), \tilde{v}_{\tilde{P}}^{L}(z)\right\}$,

$\tilde{v}_{\tilde{Q}}^{U}(y z) \geq \max \left\{\tilde{v}_{\tilde{P}}^{U}(y), \tilde{v}_{\tilde{P}}^{U}(z)\right\}$

and $\left(\tilde{\mu}_{\tilde{Q}}^{U}(y z)\right)^{2}+\left(\tilde{v}_{\tilde{Q}}^{U}(y z)\right)^{2} \leq 1$ for all $y, z \in Z$.

Here $\tilde{P}$ is the interval-valued Pythagorean fuzzy vertex set of $\tilde{\mathcal{G}}$ and $\tilde{Q}$ is the interval-valued Pythagorean fuzzy edge set of $\tilde{\mathcal{G}}$.

Example 1 Consider a graph $G=(V, E)$, where $V=$ $\{a, b, c, d, e, f, g\}$ is the vertex set and $E=\{a b, a c, a d, a e$, $e f, c g\}$ is the edge set of $G$. Let $\tilde{\mathcal{G}}=(\tilde{P}, \tilde{Q})$ be an IVPFG on $V$, as shown in Fig. 4, defined by:

\begin{tabular}{llllllll}
\hline$\tilde{P}$ & $a$ & $b$ & $c$ & $d$ & $e$ & $f$ & $g$ \\
\hline$\tilde{\mu}_{\tilde{P}}^{L}$ & 0.5 & 0.5 & 0.4 & 0.2 & 0.3 & 0.4 & 0.2 \\
$\tilde{\mu} \tilde{\tilde{P}}$ & 0.9 & 0.7 & 0.5 & 0.8 & 0.8 & 0.7 & 0.7 \\
$\tilde{\nu}_{\tilde{P}}^{U}$ & 0.2 & 0.1 & 0.3 & 0.1 & 0.2 & 0.1 & 0.3 \\
$\tilde{\nu}_{\tilde{P}}^{U}$ & 0.3 & 0.4 & 0.7 & 0.4 & 0.5 & 0.6 & 0.6 \\
\hline
\end{tabular}

\begin{tabular}{lllllll}
\hline$\tilde{Q}$ & $a b$ & $a c$ & $a d$ & $a e$ & ef & $c g$ \\
\hline$\tilde{\mu}_{\tilde{Q}}^{L}$ & 0.4 & 0.1 & 0.1 & 0.2 & 0.1 & 0.1 \\
$\tilde{\mu}_{\tilde{Q}}^{U}$ & 0.5 & 0.4 & 0.7 & 0.4 & 0.6 & 0.5 \\
$\tilde{v}_{\tilde{Q}}^{L}$ & 0.6 & 0.6 & 0.3 & 0.3 & 0.3 & 0.4 \\
$\tilde{v}_{\tilde{Q}}^{U}$ & 0.7 & 0.8 & 0.7 & 0.8 & 0.8 & 0.8 \\
\hline
\end{tabular}

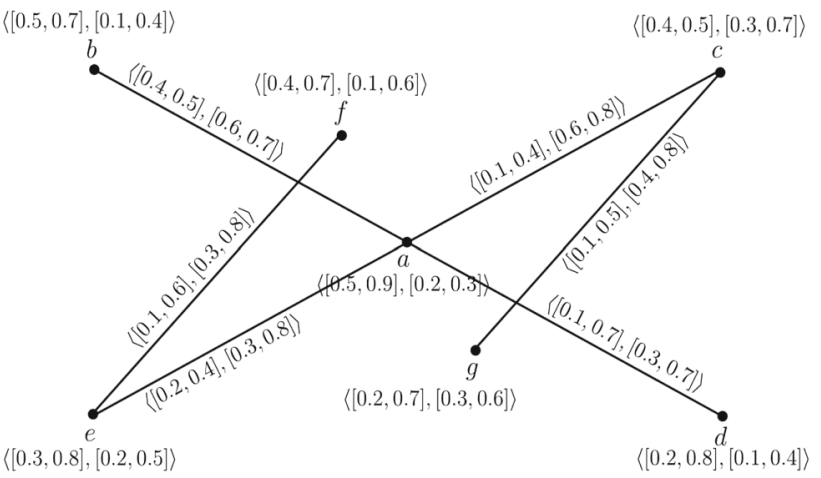

Fig. 4 The interval-valued Pythagorean fuzzy graph

By direct computation, it is very easy to see from Fig. 4 that $\tilde{\mathcal{G}}=(\tilde{P}, \tilde{Q})$ ia an IVPFG.

Definition 9 A SIVPFS $\tilde{\mathcal{Q}}$ in $Z \times Z$ is said to be a simplified interval-valued Pythagorean fuzzy relation in $Z$, denoted by

$\tilde{\mathcal{Q}}=\{\langle y z, \dot{\zeta}, \dot{\eta}\rangle \mid y z \in Z \times Z\}$,

where $\dot{\zeta}=\left(\tilde{\mu}_{\tilde{\mathcal{Q}}}^{L}(y z), \tilde{v}_{\tilde{\mathcal{Q}}}^{U}(y z)\right)$ and $\dot{\eta}=\left(\tilde{\mu}_{\tilde{\mathcal{Q}}}^{U}(y z), \tilde{v}_{\tilde{\mathcal{Q}}}^{L}(y z)\right)$ such that $\dot{\zeta} \cup \dot{\eta}=\dot{\eta}$ (or $\dot{\zeta} \cap \dot{\eta}=\dot{\zeta}) . \tilde{\mu}_{\tilde{\mathcal{Q}}}^{L}: Z \times Z \rightarrow$ $[0,1], \tilde{\mu}_{\tilde{\mathcal{Q}}}^{U}: Z \times Z \rightarrow[0,1]$ and $\tilde{\nu}_{\tilde{\mathcal{Q}}}^{L}: Z \times Z \rightarrow$ $[0,1], \tilde{v}_{\tilde{\mathcal{Q}}}^{U}: Z \times Z \rightarrow[0,1]$ represent the membership and non-membership functions of $\tilde{\mathcal{Q}}$, respectively, for all $y, z \in Z$.

Definition 10 A SIVPFG on a non-empty set $Z$ is a pair $\tilde{\mathcal{G}}=$ $(\tilde{\mathcal{P}}, \tilde{\mathcal{Q}})$, where $\tilde{\mathcal{P}}$ is a SIVPFS on $Z$ and $\tilde{\mathcal{Q}}$ is a SIVPFR on $Z$ such that

$\tilde{\mu}_{\tilde{\mathcal{Q}}}^{L}(y z) \leq \min \left\{\tilde{\mu}_{\tilde{\mathcal{P}}}^{L}(y), \tilde{\mu}_{\tilde{\mathcal{P}}}^{L}(z)\right\}, \quad \tilde{v}_{\tilde{\mathcal{Q}}}^{U}(y z) \geq \max \left\{\tilde{v}_{\tilde{\mathcal{P}}}^{U}(y), \tilde{v}_{\tilde{\mathcal{P}}}^{U}(z)\right\}$,

$\tilde{\mu}_{\tilde{\mathcal{Q}}}^{U}(y z) \leq \min \left\{\tilde{\mu}_{\tilde{\mathcal{P}}}^{U}(y), \tilde{\mu}_{\tilde{\mathcal{P}}}^{U}(z)\right\}, \quad \tilde{v}_{\tilde{\mathcal{Q}}}^{L}(y z) \geq \max \left\{\tilde{v}_{\tilde{\mathcal{P}}}^{L}(y), \tilde{v}_{\tilde{\mathcal{P}}}^{L}(z)\right\}$

and $\dot{\zeta} \cup \dot{\eta}=\dot{\eta}$ (or $\dot{\zeta} \cap \dot{\eta}=\dot{\zeta}$ ) for all $y, z \in Z$, where $\dot{\zeta}=\left(\tilde{\mu}_{\tilde{\mathcal{P}}}^{L}(y z), \tilde{v}_{\tilde{\mathcal{P}}}^{U}(y z)\right)$ and $\dot{\eta}=\left(\tilde{\mu}_{\tilde{\mathcal{P}}}^{U}(y z), \tilde{v}_{\tilde{\mathcal{P}}}^{L}(y z)\right)$.

Here $\tilde{\mathcal{P}}$ and $\tilde{\mathcal{Q}}$ are the simplified interval-valued Pythagorean fuzzy vertex set and the simplified intervalvalued Pythagorean fuzzy edge set of $\tilde{\mathcal{G}}$, respectively. $\tilde{\mathcal{Q}}$ is a symmetric simplified interval-valued Pythagorean fuzzy relation on $\tilde{\mathcal{P}}$. If $\tilde{\mathcal{Q}}$ is not symmetric on $\tilde{\mathcal{P}}$, then $\tilde{\mathcal{D}}=(\tilde{\mathcal{P}}, \overrightarrow{\tilde{\mathcal{Q}}})$ 


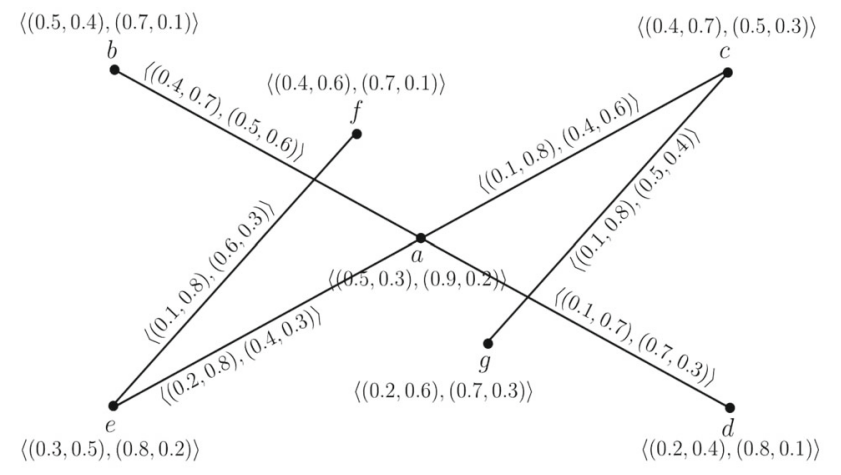

Fig. 5 The simplified interval-valued Pythagorean fuzzy graph

is called a simplified interval-valued Pythagorean fuzzy digraph.

Example 2 Consider an IVPFG $\tilde{\mathcal{G}}=(\tilde{P}, \tilde{Q})$ on $V=$ $\{a, b, c, d, e, f, g\}$, as shown in Fig. 4. By simplifying the expression way of this IVPFG, let $\tilde{\mathcal{G}}=(\tilde{\mathcal{P}}, \tilde{\mathcal{Q}})$ be a SIVPFG on $V$, as shown in Fig. 5. By direct computation, it is very easy to see from Fig. 5 that $\tilde{\mathcal{G}}=(\tilde{\mathcal{P}}, \tilde{\mathcal{Q}})$ is a SIVPFG.

Definition 11 The degree and total degree of a vertex $z \in Z$ in a SIVPFG $\tilde{\mathcal{G}}$ is defined as $\mathrm{d}_{\tilde{\mathcal{G}}}(z)=\left\langle\left(\mathrm{d}_{\tilde{\mu}^{L}}(z), \mathrm{d}_{\tilde{v}^{U}}(z)\right)\right.$, $\left.\left(\mathrm{d}_{\tilde{\mu}^{U}}(z), \mathrm{d}_{\tilde{v}^{L}}(z)\right)\right\rangle$ and $\operatorname{td}_{\tilde{\mathcal{G}}}(z)=\left\langle\left(\operatorname{td}_{\tilde{\mu}^{L}}(z), \operatorname{td}_{\tilde{v}}(z)\right)\right.$, $\left.\left(\operatorname{td}_{\tilde{\mu}}{ }^{U}(z), \operatorname{td}_{\tilde{v}} L(z)\right)\right\rangle$, respectively, where

$$
\begin{aligned}
& \mathrm{d}_{\tilde{\mu}^{L}}(z)=\sum_{y, z \neq y \in Z} \tilde{\mu}_{\tilde{\mathcal{Q}}^{L}}^{L}(y z), \mathrm{d}_{\tilde{v} U}(z)=\sum_{y, z \neq y \in Z} \tilde{v}_{\tilde{\mathcal{Q}}}^{U}(y z),
\end{aligned}
$$

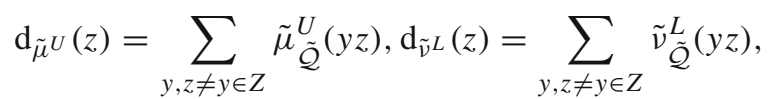

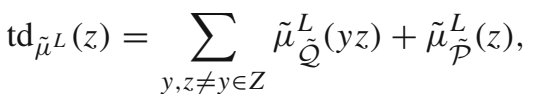

$$
\begin{aligned}
& \operatorname{td}_{\tilde{\nu}}(z)=\sum_{y, z \neq y \in Z} \tilde{v}_{\tilde{\mathcal{Q}}}^{U}(y z)+\tilde{v}_{\tilde{\mathcal{P}}}^{U}(z),
\end{aligned}
$$

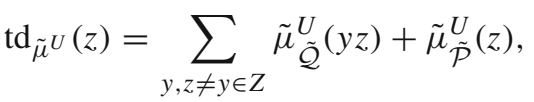

$$
\begin{aligned}
& \operatorname{td}_{\tilde{v} L}(z)=\sum_{y, z \neq y \in Z} \tilde{v}_{\tilde{\mathcal{Q}}}^{L}(y z)+\tilde{v}_{\tilde{\mathcal{P}}}^{L}(z) .
\end{aligned}
$$

Example 3 The degree of a vertex $a$ in SIVPFG $\tilde{\mathcal{G}}$ shown in Fig. 5 , is $\mathrm{d}_{\tilde{\mathcal{G}}}(a)=\langle(0.8,3.0),(0.2,1.8)\rangle$, whereas the total degree of a vertex $a$ is $t \mathrm{~d}_{\tilde{\mathcal{G}}}(a)=\langle(1.3,3.3),(2.9,2.0)\rangle$.

There are many operations on two graphs $G_{1}=\left(V_{1}, E_{1}\right)$ and $G_{2}=\left(V_{2}, E_{2}\right)\left(\left|V_{i}\right|=r_{i}, i=1,2\right)$ which result in a graph whose vertex set is the Cartesian product $V_{1} \times V_{2}$. We discuss a few operations on two graphs in the structure of SIVPFS theory and investigate their properties.
Definition 12 Let $\tilde{\mathcal{G}}_{1}=\left(\tilde{\mathcal{P}}_{1}, \tilde{\mathcal{Q}}_{1}\right)$ and $\tilde{\mathcal{G}}_{2}=\left(\tilde{\mathcal{P}}_{2}, \tilde{\mathcal{Q}}_{2}\right)$ be two SIVPFGs of the graphs $G_{1}=\left(V_{1}, E_{1}\right)$ and $G_{2}=\left(V_{2}, E_{2}\right)$, respectively. The tensor product or conjunction of $\tilde{\mathcal{G}}_{1}$ and $\tilde{\mathcal{G}}_{2}$ is denoted by $\tilde{\mathcal{G}}_{1} \times \tilde{\mathcal{G}}_{2}=\left(\tilde{\mathcal{P}}_{1} \times \tilde{\mathcal{P}}_{2}, \tilde{\mathcal{Q}}_{1} \times \tilde{\mathcal{Q}}_{2}\right)$ and defined as:

$$
\left\{\begin{array}{l}
\left(\tilde{\mu}_{\tilde{\mathcal{P}}_{1}}^{L} \times \tilde{\mu}_{\tilde{\mathcal{P}}_{2}}^{L}\right)\left(z_{1}, z_{2}\right)=\tilde{\mu}_{\tilde{\mathcal{P}}_{1}}^{L}\left(z_{1}\right) \wedge \tilde{\mu}_{\tilde{\mathcal{P}}_{2}}^{L}\left(z_{2}\right) \\
\left(\tilde{v}_{\tilde{\mathcal{P}}_{1}}^{U} \times \tilde{v}_{\tilde{\mathcal{P}}_{2}}^{U}\right)\left(z_{1}, z_{2}\right)=\tilde{v}_{\tilde{\mathcal{P}}_{1}}^{U}\left(z_{1}\right) \vee \tilde{v}_{\tilde{\mathcal{P}}_{2}}^{U}\left(z_{2}\right) \\
\left(\tilde{\mu}_{\tilde{\mathcal{P}}_{1}}^{U} \times \tilde{\mu}_{\tilde{\mathcal{P}}_{2}}^{U}\right)\left(z_{1}, z_{2}\right)=\tilde{\mu}_{\tilde{\mathcal{P}}_{1}}^{U}\left(z_{1}\right) \wedge \tilde{\mu}_{\tilde{\mathcal{P}}_{2}}^{U}\left(z_{2}\right) \\
\left(\tilde{v}_{\tilde{\mathcal{P}}_{1}}^{L} \times \tilde{v}_{\tilde{\mathcal{P}}_{2}}^{L}\right)\left(z_{1}, z_{2}\right)=\tilde{v}_{\tilde{\mathcal{P}}_{1}}^{L}\left(z_{1}\right) \vee \tilde{v}_{\tilde{\mathcal{P}}_{2}}^{L}\left(z_{2}\right) \\
\quad \text { for all }\left(z_{1}, z_{2}\right) \in V_{1} \times V_{2},
\end{array}\right.
$$$$
\text { (ii) }\left\{\begin{array}{c}
\left(\tilde{\mu}_{\tilde{\mathcal{Q}}_{1}}^{L} \times \tilde{\mu}_{\tilde{\mathcal{Q}}_{2}}^{L}\right)\left(\left(y_{1}, y_{2}\right)\left(z_{1}, z_{2}\right)\right) \\
=\tilde{\mu}_{\tilde{\mathcal{P}}_{1}}^{L}\left(y_{1} z_{1}\right) \wedge \tilde{\mu}_{\tilde{\mathcal{Q}}_{2}}^{L}\left(y_{2} z_{2}\right) \\
\left(\tilde{v}_{\tilde{\mathcal{Q}}_{1}}^{U} \times \tilde{v}_{\tilde{\mathcal{Q}}_{2}}^{U}\right)\left(\left(y_{1}, y_{2}\right)\left(z_{1}, z_{2}\right)\right) \\
=\tilde{v}_{\tilde{\mathcal{P}}_{1}}^{U}\left(y_{1} z_{1}\right) \vee \tilde{v}_{\tilde{\mathcal{Q}}_{2}}^{U}\left(y_{2} z_{2}\right) \\
\left(\tilde{\mu}_{\tilde{\mathcal{Q}}_{1}}^{U} \times \tilde{\mu}_{\tilde{\mathcal{Q}}_{2}}^{U}\right)\left(\left(y_{1}, y_{2}\right)\left(z_{1}, z_{2}\right)\right) \\
=\tilde{\mu}_{\tilde{\mathcal{P}}_{1}}^{U}\left(y_{1} z_{1}\right) \wedge \tilde{\mu}_{\tilde{\mathcal{Q}}_{2}}^{U}\left(y_{2} z_{2}\right) \\
\left(\tilde{v}_{\tilde{\mathcal{Q}}_{1}}^{L} \times \tilde{v}_{\tilde{\mathcal{Q}}_{2}}^{L}\right)\left(\left(y_{1}, y_{2}\right)\left(z_{1}, z_{2}\right)\right) \\
=\tilde{v}_{\tilde{\mathcal{P}}_{1}}^{L}\left(y_{1} z_{1}\right) \vee \tilde{v}_{\tilde{\mathcal{Q}}_{2}}^{L}\left(y_{2} z_{2}\right) \\
\text { for all } y_{1} z_{1} \in E_{1}, y_{2} z_{2} \in E_{2} .
\end{array}\right.
$$

Proposition 1 Let $\tilde{\mathcal{G}}_{1}$ and $\tilde{\mathcal{G}}_{2}$ be two SIVPFGs of the graphs $G_{1}$ and $G_{2}$, respectively. The tensor product $\tilde{\mathcal{G}}_{1} \times \tilde{\mathcal{G}}_{2}$ of $\tilde{\mathcal{G}}_{1}$ and $\tilde{\mathcal{G}}_{2}$ is a SIVPFG of $G_{1} \times G_{2}$.

Definition 13 Let $\tilde{\mathcal{G}}_{1}=\left(\tilde{\mathcal{P}}_{1}, \tilde{\mathcal{Q}}_{1}\right)$ and $\tilde{\mathcal{G}}_{2}=\left(\tilde{\mathcal{P}}_{2}, \tilde{\mathcal{Q}}_{2}\right)$ be two SIVPFGs. For any vertex $\left(z_{1}, z_{2}\right) \in V_{1} \times V_{2}$,

$$
\begin{aligned}
& \left(\mathrm{d}_{\tilde{\mu}^{L}}\right)_{\tilde{\mathcal{G}}_{1} \times \tilde{\mathcal{G}}_{2}}\left(z_{1}, z_{2}\right) \\
& =\sum_{\left(y_{1}, y_{2}\right)\left(z_{1}, z_{2}\right) \in E_{1} \times E_{2}}\left(\tilde{\mu}_{\tilde{\mathcal{Q}}_{1}}^{L} \times \tilde{\mu}_{\tilde{\mathcal{Q}}_{2}}^{L}\right)\left(\left(y_{1}, y_{2}\right)\left(z_{1}, z_{2}\right)\right) \\
& =\sum_{y_{1} z_{1} \in E_{1}, y_{2} z_{2} \in E_{2}} \tilde{\mu}_{\tilde{\mathcal{Q}}_{1}}^{L}\left(y_{1} z_{1}\right) \wedge \tilde{\mu}_{\tilde{\mathcal{Q}}_{2}}^{L}\left(y_{2} z_{2}\right), \\
& \left(\mathrm{d}_{\tilde{v} U}\right)_{\tilde{\mathcal{G}}_{1} \times \tilde{\mathcal{G}}_{2}}\left(z_{1}, z_{2}\right) \\
& =\sum_{\left(y_{1}, y_{2}\right)\left(z_{1}, z_{2}\right) \in E_{1} \times E_{2}}\left(\tilde{v}_{\tilde{\mathcal{Q}}_{1}}^{U} \times \tilde{v}_{\tilde{\mathcal{Q}}_{2}}^{U}\right)\left(\left(y_{1}, y_{2}\right)\left(z_{1}, z_{2}\right)\right) \\
& =\sum_{y_{1} z_{1} \in E_{1}, y_{2} z_{2} \in E_{2}} \tilde{v}_{\tilde{\mathcal{Q}}_{1}}^{U}\left(y_{1} z_{1}\right) \vee \tilde{v}_{\tilde{\mathcal{Q}}_{2}}^{U}\left(y_{2} z_{2}\right), \\
& \left(\mathrm{d}_{\tilde{\mu}^{U}}\right)_{\tilde{\mathcal{G}}_{1} \times \tilde{\mathcal{G}}_{2}}\left(z_{1}, z_{2}\right) \\
& =\sum_{\left(y_{1}, y_{2}\right)\left(z_{1}, z_{2}\right) \in E_{1} \times E_{2}}\left(\tilde{\mu}_{\tilde{\mathcal{Q}}_{1}}^{U} \times \tilde{\mu}_{\tilde{\mathcal{Q}}_{2}}^{U}\right)\left(\left(y_{1}, y_{2}\right)\left(z_{1}, z_{2}\right)\right) \\
& =\sum_{y_{1} z_{1} \in E_{1}, y_{2} z_{2} \in E_{2}} \tilde{\mu}_{\tilde{\mathcal{Q}}_{1}}^{U}\left(y_{1} z_{1}\right) \wedge \tilde{\mu}_{\tilde{\mathcal{Q}}_{2}}^{U}\left(y_{2} z_{2}\right)
\end{aligned}
$$




$$
\begin{aligned}
& \left(\mathrm{d}_{\left.\tilde{v}^{L}\right)}\right)_{\tilde{\mathcal{G}}_{1} \times \tilde{\mathcal{G}}_{2}}\left(z_{1}, z_{2}\right) \\
& =\sum_{\left(y_{1}, y_{2}\right)\left(z_{1}, z_{2}\right) \in E_{1} \times E_{2}}\left(\tilde{v}_{\tilde{\mathcal{Q}}_{1}}^{L} \times \tilde{v}_{\tilde{\mathcal{Q}}_{2}}^{L}\right)\left(\left(y_{1}, y_{2}\right)\left(z_{1}, z_{2}\right)\right) \\
& =\sum_{y_{1} z_{1} \in E_{1}, y_{2} z_{2} \in E_{2}} \tilde{v}_{\tilde{\mathcal{Q}}_{1}}^{L}\left(y_{1} z_{1}\right) \vee \tilde{v}_{\tilde{\mathcal{Q}}_{2}}^{L}\left(y_{2} z_{2}\right) .
\end{aligned}
$$

Theorem 2 Let $\tilde{\mathcal{G}}_{1}$ and $\tilde{\mathcal{G}}_{2}$ be two SIVPFGs. If $\tilde{\mu}_{\tilde{\mathcal{Q}}_{2}}^{L} \geq$ $\tilde{\mu}_{\tilde{\mathcal{Q}}_{1}}^{L}, \tilde{v}_{\tilde{\mathcal{Q}}_{2}}^{U} \leq \tilde{v}_{\tilde{\mathcal{Q}}_{1}}^{U}, \tilde{\mu}_{\tilde{\mathcal{Q}}_{2}}^{U} \geq \tilde{\mu}_{\tilde{\mathcal{Q}}_{1}}^{U}, \tilde{v}_{\tilde{\mathcal{Q}}_{2}}^{L} \leq \tilde{v}_{\tilde{\mathcal{Q}}_{1}}^{L}$, then $\mathrm{d}_{\tilde{\mathcal{G}}_{1} \times \tilde{\mathcal{G}}_{2}}\left(z_{1}, z_{2}\right)=\mathrm{d}_{\tilde{\mathcal{G}}_{1}}\left(z_{1}\right)$ and if $\tilde{\mu}_{\tilde{\mathcal{Q}}_{1}}^{L} \geq \tilde{\mu}_{\tilde{\mathcal{Q}}_{2}}^{L}, \tilde{v}_{\tilde{\mathcal{Q}}_{1}}^{U} \leq$ $\tilde{v}_{\tilde{\mathcal{Q}}_{2}}^{U}, \tilde{\mu}_{\tilde{\mathcal{Q}}_{1}}^{U} \geq \tilde{\mu}_{\tilde{\mathcal{Q}}_{2}}^{U}, \tilde{v}_{\tilde{\mathcal{Q}}_{1}}^{L} \leq \tilde{v}_{\tilde{\mathcal{Q}}_{2}}^{L}$, then $\mathrm{d}_{\tilde{\mathcal{G}}_{1} \times \tilde{\mathcal{G}}_{2}}\left(z_{1}, z_{2}\right)=$ $\mathrm{d}_{\tilde{\mathcal{G}}_{2}}\left(z_{2}\right)$ for all $\left(z_{1}, z_{2}\right) \in V_{1} \times V_{2}$.

Proof By definition of vertex degree of $\tilde{\mathcal{G}}_{1} \times \tilde{\mathcal{G}}_{2}$, we have

$$
\begin{aligned}
& \left(\mathrm{d}_{\tilde{\mu}^{L}}\right)_{\tilde{\mathcal{G}}_{1} \times \tilde{\mathcal{G}}_{2}}\left(z_{1}, z_{2}\right) \\
& =\sum_{\left(y_{1}, y_{2}\right)\left(z_{1}, z_{2}\right) \in E_{1} \times E_{2}}\left(\tilde{\mu}_{\tilde{\mathcal{Q}}_{1}}^{L} \times \tilde{\mu}_{\tilde{\mathcal{Q}}_{2}}^{L}\right)\left(\left(y_{1}, y_{2}\right)\left(z_{1}, z_{2}\right)\right) \\
& =\sum_{y_{1} z_{1} \in E_{1}, y_{2} z_{2} \in E_{2}} \tilde{\mu}_{\tilde{\mathcal{Q}}_{1}}^{L}\left(y_{1} z_{1}\right) \wedge \tilde{\mu}_{\tilde{\mathcal{Q}}_{2}}^{L}\left(y_{2} z_{2}\right) \\
& =\sum_{y_{1} z_{1} \in E_{1}} \tilde{\mu}_{\tilde{\mathcal{Q}}_{1}}^{L}\left(y_{1} z_{1}\right) \quad\left(\text { since } \tilde{\mu}_{\tilde{\mathcal{Q}}_{2}}^{L} \geq \tilde{\mu}_{\tilde{\mathcal{Q}}_{1}}^{L}\right) \\
& =\left(\mathrm{d}_{\left.\tilde{\mu}^{L}\right)} \tilde{\mathcal{G}}_{1}\left(z_{1}\right),\right. \\
& \left(\mathrm{d}_{\tilde{v}^{U}}\right)_{\tilde{\mathcal{G}}_{1} \times \tilde{\mathcal{G}}_{2}}\left(z_{1}, z_{2}\right) \\
& =\sum_{\left(y_{1}, y_{2}\right)\left(z_{1}, z_{2}\right) \in E_{1} \times E_{2}}\left(\tilde{v}_{\tilde{\mathcal{Q}}_{1}}^{U} \times \tilde{v}_{\tilde{\mathcal{Q}}_{2}}^{U}\right)\left(\left(y_{1}, y_{2}\right)\left(z_{1}, z_{2}\right)\right) \\
& =\sum_{y_{1} z_{1} \in E_{1}, y_{2} z_{2} \in E_{2}} \tilde{v}_{\tilde{\mathcal{Q}}_{1}}^{U}\left(y_{1} z_{1}\right) \vee \tilde{v}_{\tilde{\mathcal{Q}}_{2}}^{U}\left(y_{2} z_{2}\right) \\
& =\sum_{y_{1} z_{1} \in E_{1}} \tilde{v}_{\tilde{\mathcal{Q}}_{1}}^{U}\left(y_{1} z_{1}\right) \quad\left(\text { since } \tilde{v}_{\tilde{\mathcal{Q}}_{2}}^{U} \leq \tilde{v}_{\tilde{\mathcal{Q}}_{1}}^{U}\right) \\
& =\left(\mathrm{d}_{\tilde{v} U}\right)_{\tilde{\mathcal{G}}_{1}}\left(z_{1}\right), \\
& \left(\mathrm{d}_{\tilde{\mu}^{U}}\right)_{\tilde{\mathcal{G}}_{1} \times \tilde{\mathcal{G}}_{2}}\left(z_{1}, z_{2}\right) \\
& =\sum_{\left(y_{1}, y_{2}\right)\left(z_{1}, z_{2}\right) \in E_{1} \times E_{2}}\left(\tilde{\mu}_{\tilde{\mathcal{Q}}_{1}}^{U} \times \tilde{\mu}_{\tilde{\mathcal{Q}}_{2}}^{U}\right)\left(\left(y_{1}, y_{2}\right)\left(z_{1}, z_{2}\right)\right) \\
& =\sum_{y_{1} z_{1} \in E_{1}, y_{2} z_{2} \in E_{2}} \tilde{\mu}_{\tilde{\mathcal{Q}}_{1}}^{U}\left(y_{1} z_{1}\right) \wedge \tilde{\mu}_{\tilde{\mathcal{Q}}_{2}}^{U}\left(y_{2} z_{2}\right) \\
& =\sum_{y_{1} z_{1} \in E_{1}} \tilde{\mu}_{\tilde{\mathcal{Q}}_{1}}^{U}\left(y_{1} z_{1}\right) \quad\left(\text { since } \tilde{\mu}_{\tilde{\mathcal{Q}}_{2}}^{U} \geq \tilde{\mu}_{\tilde{\mathcal{Q}}_{1}}^{U}\right) \\
& =\left(\mathrm{d}_{\tilde{\mu}^{U}}\right)_{\tilde{\mathcal{G}}_{1}}\left(z_{1}\right) \text {, } \\
& \left(\mathrm{d}_{\tilde{v}^{L}}\right)_{\tilde{\mathcal{G}}_{1} \times \tilde{\mathcal{G}}_{2}}\left(z_{1}, z_{2}\right) \\
& =\sum_{\left(y_{1}, y_{2}\right)\left(z_{1}, z_{2}\right) \in E_{1} \times E_{2}}\left(\tilde{v}_{\tilde{\mathcal{Q}}_{1}}^{L} \times \tilde{v}_{\tilde{\mathcal{Q}}_{2}}^{L}\right)\left(\left(y_{1}, y_{2}\right)\left(z_{1}, z_{2}\right)\right) \\
& =\sum_{y_{1} z_{1} \in E_{1}, y_{2} z_{2} \in E_{2}} \tilde{v}_{\tilde{\mathcal{Q}}_{1}}^{L}\left(y_{1} z_{1}\right) \vee \tilde{v}_{\tilde{\mathcal{Q}}_{2}}^{L}\left(y_{2} z_{2}\right)
\end{aligned}
$$

$$
\begin{aligned}
& =\sum_{y_{1} z_{1} \in E_{1}} \tilde{v}_{\tilde{\mathcal{Q}}_{1}}^{L}\left(y_{1} z_{1}\right) \quad\left(\text { since } \tilde{v}_{\tilde{\mathcal{Q}}_{2}}^{L} \leq \tilde{v}_{\tilde{\mathcal{Q}}_{1}}^{L}\right) \\
& =\left(\mathrm{d}_{\tilde{v}^{L}}\right)_{\tilde{\mathcal{G}}_{1}}\left(z_{1}\right) .
\end{aligned}
$$

Hence $\mathrm{d}_{\tilde{\mathcal{G}}_{1} \times \tilde{\mathcal{G}}_{2}}\left(z_{1}, z_{2}\right)=\mathrm{d}_{\tilde{\mathcal{G}}_{1}}\left(z_{1}\right)$. Similarly, it is easy to show that, if $\tilde{\mu}_{\tilde{\mathcal{Q}}_{1}}^{L} \geq \tilde{\mu}_{\tilde{\mathcal{Q}}_{2}}^{L}, \tilde{v}_{\tilde{\mathcal{Q}}_{1}}^{U} \leq \tilde{v}_{\tilde{\mathcal{Q}}_{2}}^{U}, \tilde{\mu}_{\tilde{\mathcal{Q}}_{1}}^{U} \geq \tilde{\mu}_{\tilde{\mathcal{Q}}_{2}}^{U}, \tilde{v}_{\tilde{\mathcal{Q}}_{1}}^{L} \leq \tilde{v}_{\tilde{\mathcal{Q}}_{2}}^{L}$, then $\mathrm{d}_{\tilde{\mathcal{G}}_{1} \times \tilde{\mathcal{G}}_{2}}\left(z_{1}, z_{2}\right)=\mathrm{d}_{\tilde{\mathcal{G}}_{2}}\left(z_{2}\right)$.

Definition 14 Let $\tilde{\mathcal{G}}_{1}$ and $\tilde{\mathcal{G}}_{2}$ be two SIVPFGs. For any vertex $\left(z_{1}, z_{2}\right) \in V_{1} \times V_{2}$,

$$
\begin{aligned}
& \left(t \mathrm{~d}_{\tilde{\mu}^{L}}\right)_{\tilde{\mathcal{G}}_{1} \times \tilde{\mathcal{G}}_{2}}\left(z_{1}, z_{2}\right) \\
& =\sum_{\left(y_{1}, y_{2}\right)\left(z_{1}, z_{2}\right) \in E_{1} \times E_{2}}\left(\tilde{\mu}_{\tilde{\mathcal{Q}}_{1}}^{L} \times \tilde{\mu}_{\tilde{\mathcal{Q}}_{2}}^{L}\right)\left(\left(y_{1}, y_{2}\right)\left(z_{1}, z_{2}\right)\right) \\
& +\left(\tilde{\mu}_{\tilde{\mathcal{P}}_{1}}^{L} \times \tilde{\mu}_{\tilde{\mathcal{P}}_{2}}^{L}\right)\left(z_{1}, z_{2}\right), \\
& \left(t \mathrm{~d}_{\tilde{\nu} U}\right)_{\tilde{\mathcal{G}}_{1} \times \tilde{\mathcal{G}}_{2}}\left(z_{1}, z_{2}\right) \\
& =\sum_{\left(y_{1}, y_{2}\right)\left(z_{1}, z_{2}\right) \in E_{1} \times E_{2}}\left(\tilde{v}_{\tilde{\mathcal{Q}}_{1}}^{U} \times \tilde{v}_{\tilde{\mathcal{Q}}_{2}}^{U}\right)\left(\left(y_{1}, y_{2}\right)\left(z_{1}, z_{2}\right)\right) \\
& +\left(\tilde{v}_{\tilde{\mathcal{P}}_{1}}^{U} \times \tilde{v}_{\tilde{\mathcal{P}}_{2}}^{U}\right)\left(z_{1}, z_{2}\right), \\
& \left(t \mathrm{~d}_{\tilde{\mu}^{U}} \tilde{\mathcal{G}}_{1} \times \tilde{\mathcal{G}}_{2}\left(z_{1}, z_{2}\right)\right. \\
& =\sum_{\left(y_{1}, y_{2}\right)\left(z_{1}, z_{2}\right) \in E_{1} \times E_{2}}\left(\tilde{\mu}_{\tilde{\mathcal{Q}}_{1}}^{U} \times \tilde{\mu}_{\tilde{\mathcal{Q}}_{2}}^{U}\right)\left(\left(y_{1}, y_{2}\right)\left(z_{1}, z_{2}\right)\right) \\
& +\left(\tilde{\mu}_{\tilde{\mathcal{P}}_{1}}^{U} \times \tilde{\mu}_{\tilde{\mathcal{P}}_{2}}^{U}\right)\left(z_{1}, z_{2}\right), \\
& \left(t \mathrm{~d}_{\tilde{v} L}\right)_{\tilde{\mathcal{G}}_{1} \times \tilde{\mathcal{G}}_{2}}\left(z_{1}, z_{2}\right) \\
& =\sum_{\left(y_{1}, y_{2}\right)\left(z_{1}, z_{2}\right) \in E_{1} \times E_{2}}\left(\tilde{v}_{\tilde{\mathcal{Q}}_{1}}^{L} \times \tilde{v}_{\tilde{\mathcal{Q}}_{2}}^{L}\right)\left(\left(y_{1}, y_{2}\right)\left(z_{1}, z_{2}\right)\right) \\
& +\left(\tilde{v}_{\tilde{\mathcal{P}}_{1}}^{L} \times \tilde{v}_{\tilde{\mathcal{P}}_{2}}^{L}\right)\left(z_{1}, z_{2}\right) .
\end{aligned}
$$

Theorem 3 Let $\tilde{\mathcal{G}}_{1}$ and $\tilde{\mathcal{G}}_{2}$ be two SIVPFGs. If

(i) $\tilde{\mu}_{\tilde{\mathcal{Q}}_{2}}^{L} \geq \tilde{\mu}_{\tilde{\mathcal{Q}}_{1}}^{L}$, then $\left(t \mathrm{~d}_{\tilde{\mu}^{L}}\right)_{\tilde{\mathcal{G}}_{1} \times \tilde{\mathcal{G}}_{2}}\left(z_{1}, z_{2}\right)=\left(\mathrm{d}_{\tilde{\mu}^{L}}\right)_{\tilde{\mathcal{G}}_{1}}\left(z_{1}\right)+$ $\tilde{\mu}_{\tilde{\mathcal{P}}_{1}}^{L}\left(z_{1}\right) \wedge \tilde{\mu}_{\tilde{\mathcal{P}}_{2}}^{L}\left(z_{2}\right)$

(ii) $\tilde{v}_{\tilde{\mathcal{Q}}_{2}}^{U} \leq \tilde{v}_{\tilde{\mathcal{Q}}_{1}}^{U}$, then $\left(t \mathrm{~d}_{\tilde{v} U}\right)_{\tilde{\mathcal{G}}_{1} \times \tilde{\mathcal{G}}_{2}}\left(z_{1}, z_{2}\right)=\left(\mathrm{d}_{\tilde{v} U}\right)_{\tilde{\mathcal{G}}_{1}}\left(z_{1}\right)+$ $\tilde{v}_{\tilde{\mathcal{P}}_{1}}^{U}\left(z_{1}\right) \vee \tilde{v}_{\tilde{\mathcal{P}}_{2}}^{U}\left(z_{2}\right)$;

(iii) $\tilde{\mu}_{\tilde{\mathcal{Q}}_{2}}^{U} \geq \tilde{\mu}_{\tilde{\mathcal{Q}}_{1}}^{U}$, then $\left(t \mathrm{~d}_{\tilde{\mu}^{U}}\right)_{\tilde{\mathcal{G}}_{1} \times \tilde{\mathcal{G}}_{2}}\left(z_{1}, z_{2}\right)=\left(\mathrm{d}_{\tilde{\mu}^{U}}\right)_{\tilde{\mathcal{G}}_{1}}\left(z_{1}\right)+$ $\tilde{\mu}_{\tilde{\mathcal{P}}_{1}}^{U}\left(z_{1}\right) \wedge \tilde{\mu}_{\tilde{\mathcal{P}}_{2}}^{U}\left(z_{2}\right)$

(iv) $\tilde{v}_{\tilde{\mathcal{Q}}_{2}}^{L} \leq \tilde{v}_{\tilde{\mathcal{Q}}_{1}}^{L}$, then $\left(t \mathrm{~d}_{\tilde{v}^{L}}\right)_{\tilde{\mathcal{G}}_{1} \times \tilde{\mathcal{G}}_{2}}\left(z_{1}, z_{2}\right)=\left(\mathrm{d}_{\tilde{v}^{L}}\right)_{\tilde{\mathcal{G}}_{1}}\left(z_{1}\right)+$ $\tilde{v}_{\tilde{\mathcal{P}}_{1}}^{L}\left(z_{1}\right) \vee \tilde{v}_{\tilde{\mathcal{P}}_{2}}^{L}\left(z_{2}\right)$;

(v) $\tilde{\mu}_{\tilde{\mathcal{Q}}_{1}}^{L} \geq \tilde{\mu}_{\tilde{\mathcal{Q}}_{2}}^{L}$, then $\left(t \mathrm{~d}_{\tilde{\mu}^{L}}\right)_{\tilde{\mathcal{G}}_{1} \times \tilde{\mathcal{G}}_{2}}\left(z_{1}, z_{2}\right)=\left(\mathrm{d}_{\tilde{\mu}^{L}}\right)_{\tilde{\mathcal{G}}_{2}}\left(z_{2}\right)+$ $\tilde{\mu}_{\tilde{\mathcal{P}}_{1}}^{L}\left(z_{1}\right) \wedge \tilde{\mu}_{\tilde{\mathcal{P}}_{2}}^{L}\left(z_{2}\right)$

(vi) $\tilde{v}_{\tilde{\mathcal{Q}}_{1}}^{U} \leq \tilde{v}_{\tilde{\mathcal{Q}}_{2}}^{U}$, then $\left(t \mathrm{~d}_{\tilde{v}^{U}}\right)_{\tilde{\mathcal{G}}_{1} \times \tilde{\mathcal{G}}_{2}}\left(z_{1}, z_{2}\right)=\left(\mathrm{d}_{\tilde{v}^{U}}\right)_{\tilde{\mathcal{G}}_{2}}\left(z_{2}\right)+$ $\tilde{v}_{\tilde{\mathcal{P}}_{1}}^{U}\left(z_{1}\right) \vee \tilde{v}_{\tilde{\mathcal{P}}_{2}}^{U}\left(z_{2}\right)$; 
(vii) $\tilde{\mu}_{\tilde{\mathcal{Q}}_{1}}^{U} \geq \tilde{\mu}_{\tilde{\mathcal{Q}}_{2}}^{U}$, then $\left(t \mathrm{~d}_{\tilde{\mu}^{U}}\right)_{\tilde{\mathcal{G}}_{1} \times \tilde{\mathcal{G}}_{2}}\left(z_{1}, z_{2}\right)=\left(\mathrm{d}_{\tilde{\mu}^{U}}\right)_{\tilde{\mathcal{G}}_{2}}\left(z_{2}\right)+$ $\tilde{\mu}_{\tilde{\mathcal{P}}_{1}}^{U}\left(z_{1}\right) \wedge \tilde{\mu}_{\tilde{\mathcal{P}}_{2}}^{U}\left(z_{2}\right)$

(viii) $\tilde{v}_{\tilde{\mathcal{Q}}_{1}}^{L} \leq \tilde{v}_{\tilde{\mathcal{Q}}_{2}}^{L}$, then $\left(t \mathrm{~d}_{\tilde{v}^{L}}\right)_{\tilde{\mathcal{G}}_{1} \times \tilde{\mathcal{G}}_{2}}\left(z_{1}, z_{2}\right)=\left(\mathrm{d}_{\tilde{v}^{L}}\right)_{\tilde{\mathcal{G}}_{2}}\left(z_{2}\right)+$ $\tilde{v}_{\tilde{\mathcal{P}}_{1}}^{L}\left(z_{1}\right) \vee \tilde{v}_{\tilde{\mathcal{P}}_{2}}^{L}\left(z_{2}\right)$

for all $\left(z_{1}, z_{2}\right) \in V_{1} \times V_{2}$.

Proof The proof is straightforward using Definition 14 and Theorem 2.

Definition 15 Let $\tilde{\mathcal{G}}_{1}$ and $\tilde{\mathcal{G}}_{2}$ be two SIVPFGs of $G_{1}$ and $G_{2}$, respectively. The Cartesian product of $\tilde{\mathcal{G}}_{1}$ and $\tilde{\mathcal{G}}_{2}$ is denoted by $\tilde{\mathcal{G}}_{1} \square \tilde{\mathcal{G}}_{2}=\left(\tilde{\mathcal{P}}_{1} \square \tilde{\mathcal{P}}_{2}, \tilde{\mathcal{Q}}_{1} \square \tilde{\mathcal{Q}}_{2}\right)$ and defined as:

(i)

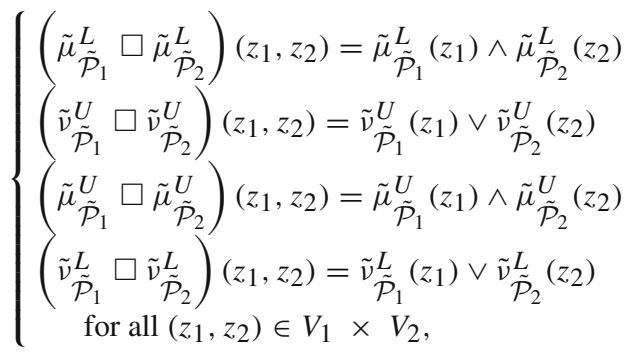$$
\left[\begin{array}{l}
\left(\tilde{\mu}_{\tilde{\mathcal{Q}}_{1}}^{L} \square \tilde{\mu}_{\tilde{\mathcal{Q}}_{2}}^{L}\right)\left(\left(y, y_{2}\right)\left(y, z_{2}\right)\right)=\tilde{\mu}_{\tilde{\mathcal{P}}_{1}}^{L}(y) \wedge \tilde{\mu}_{\tilde{\mathcal{Q}}_{2}}^{L}\left(y_{2} z_{2}\right) \\
\left(\tilde{v}_{\tilde{\mathcal{Q}}_{1}}^{U} \square \tilde{v}_{\tilde{\mathcal{Q}}_{2}}^{U}\right)\left(\left(y, y_{2}\right)\left(y, z_{2}\right)\right)=\tilde{v}_{\tilde{\mathcal{P}}_{1}}^{U}(y) \vee \tilde{v}_{\tilde{\mathcal{Q}}_{2}}^{U}\left(y_{2} z_{2}\right)
\end{array}\right.
$$

(ii)

$$
\left\{\begin{array}{l}
\left(\tilde{\mu}_{\tilde{\mathcal{Q}}_{1}}^{U} \square \tilde{\mu}_{\tilde{\mathcal{Q}}_{2}}^{U}\right)\left(\left(y, y_{2}\right)\left(y, z_{2}\right)\right)=\tilde{\mu}_{\tilde{\mathcal{P}}_{1}}^{U}(y) \wedge \tilde{\mu}_{\tilde{\mathcal{Q}}_{2}}^{U}\left(y_{2} z_{2}\right) \\
\left(\tilde{v}_{\tilde{\mathcal{Q}}_{1}}^{L} \square \tilde{v}_{\tilde{\mathcal{Q}}_{2}}^{L}\right)\left(\left(y, y_{2}\right)\left(y, z_{2}\right)\right)=\tilde{v}_{\tilde{\mathcal{P}}_{1}}^{L}(y) \vee \tilde{v}_{\tilde{\mathcal{Q}}_{2}}^{L}\left(y_{2} z_{2}\right) \\
\quad \text { for all } y \in V_{1}, y_{2} z_{2} \in E_{2},
\end{array}\right.
$$

(iii)

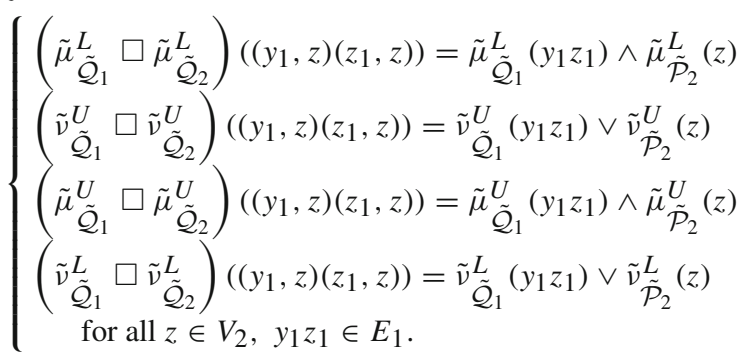

Proposition 2 The Cartesian product $\tilde{\mathcal{G}}_{1} \square \tilde{\mathcal{G}}_{2}$ of two SIVPFGs $\tilde{\mathcal{G}}_{1}$ and $\tilde{\mathcal{G}}_{2}$ is a SIVPFG.

Definition 16 Let $\tilde{\mathcal{G}}_{1}$ and $\tilde{\mathcal{G}}_{2}$ be two SIVPFGs. For any vertex $\left(z_{1}, z_{2}\right) \in V_{1} \times V_{2}$,

$$
\begin{aligned}
& \left(\mathrm{d}_{\tilde{\mu}^{L}}\right)_{\tilde{\mathcal{G}}_{1}} \square \tilde{\mathcal{G}}_{2}\left(z_{1}, z_{2}\right) \\
& =\sum_{\left(y_{1}, y_{2}\right)\left(z_{1}, z_{2}\right) \in E_{1}}^{\square E_{2}}\left(\tilde{\mu}_{\tilde{\mathcal{Q}}_{1}}^{L} \square \tilde{\mu}_{\tilde{\mathcal{Q}}_{2}}^{L}\right)\left(\left(y_{1}, y_{2}\right)\left(z_{1}, z_{2}\right)\right) \\
& =\sum_{y_{1}=z_{1}, y_{2} z_{2} \in E_{2}} \tilde{\mu}_{\tilde{\mathcal{P}}_{1}}^{L}\left(z_{1}\right) \wedge \tilde{\mu}_{\tilde{\mathcal{Q}}_{2}}^{L}\left(y_{2} z_{2}\right) \\
& \quad+\sum_{y_{2}=z_{2}, y_{1} z_{1} \in E_{1}} \tilde{\mu}_{\tilde{\mathcal{P}}_{2}}^{L}\left(z_{2}\right) \wedge \tilde{\mu}_{\tilde{\mathcal{Q}}_{1}}^{L}\left(y_{1} z_{1}\right),
\end{aligned}
$$

$$
\begin{aligned}
& \left(\mathrm{d}_{\tilde{v}^{U}}\right)_{\tilde{\mathcal{G}}_{1}} \square \tilde{\mathcal{G}}_{2}\left(z_{1}, z_{2}\right) \\
& =\sum_{\left(y_{1}, y_{2}\right)\left(z_{1}, z_{2}\right) \in E_{1}}^{\square E_{2}}\left(\tilde{v}_{\tilde{\mathcal{Q}}_{1}}^{U} \square \tilde{v}_{\tilde{\mathcal{Q}}_{2}}^{U}\right)\left(\left(y_{1}, y_{2}\right)\left(z_{1}, z_{2}\right)\right) \\
& =\sum_{y_{1}=z_{1}, y_{2} z_{2} \in E_{2}} \tilde{v}_{\tilde{\mathcal{P}}_{1}}^{U}\left(z_{1}\right) \vee \tilde{v}_{\tilde{\mathcal{Q}}_{2}}^{U}\left(y_{2} z_{2}\right) \\
& +\sum_{y_{2}=z_{2}, y_{1} z_{1} \in E_{1}} \tilde{v}_{\tilde{\mathcal{P}}_{2}}^{U}\left(z_{2}\right) \vee \tilde{v}_{\tilde{\mathcal{Q}}_{1}}^{U}\left(y_{1} z_{1}\right), \\
& \left(\mathrm{d}_{\tilde{\mu}^{U}}\right)_{\tilde{\mathcal{G}}_{1}} \square \tilde{\mathcal{G}}_{2}\left(z_{1}, z_{2}\right) \\
& =\sum_{\left(y_{1}, y_{2}\right)\left(z_{1}, z_{2}\right) \in E_{1}} \square E_{2}\left(\tilde{\mu}_{\tilde{\mathcal{Q}}_{1}}^{U} \square \tilde{\mu}_{\tilde{\mathcal{Q}}_{2}}^{U}\right)\left(\left(y_{1}, y_{2}\right)\left(z_{1}, z_{2}\right)\right) \\
& =\sum_{y_{1}=z_{1}, y_{2} z_{2} \in E_{2}} \tilde{\mu}_{\tilde{\mathcal{P}}_{1}}^{U}\left(z_{1}\right) \wedge \tilde{\mu}_{\tilde{\mathcal{Q}}_{2}}^{U}\left(y_{2} z_{2}\right) \\
& +\sum_{y_{2}=z_{2}, y_{1} z_{1} \in E_{1}} \tilde{\mu}_{\tilde{\mathcal{P}}_{2}}^{U}\left(z_{2}\right) \wedge \tilde{\mu}_{\tilde{\mathcal{Q}}_{1}}^{U}\left(y_{1} z_{1}\right), \\
& \left(\mathrm{d}_{\tilde{v}^{L}}\right)_{\tilde{\mathcal{G}}_{1}} \square \tilde{\mathcal{G}}_{2}\left(z_{1}, z_{2}\right) \\
& =\sum_{\left(y_{1}, y_{2}\right)\left(z_{1}, z_{2}\right) \in E_{1} \square E_{2}}\left(\tilde{v}_{\tilde{\mathcal{Q}}_{1}}^{L} \square \tilde{v}_{\tilde{\mathcal{Q}}_{2}}^{L}\right)\left(\left(y_{1}, y_{2}\right)\left(z_{1}, z_{2}\right)\right) \\
& =\sum_{y_{1}=z_{1}, y_{2} z_{2} \in E_{2}} \tilde{v}_{\tilde{\mathcal{P}}_{1}}^{L}\left(z_{1}\right) \vee \tilde{v}_{\tilde{\mathcal{Q}}_{2}}^{L}\left(y_{2} z_{2}\right) \\
& +\sum_{y_{2}=z_{2}, y_{1} z_{1} \in E_{1}} \tilde{v}_{\tilde{\mathcal{P}}_{2}}^{L}\left(z_{2}\right) \vee \tilde{v}_{\tilde{\mathcal{Q}}_{1}}^{L}\left(y_{1} z_{1}\right) .
\end{aligned}
$$

Theorem 4 Let $\tilde{\mathcal{G}}_{1}$ and $\tilde{\mathcal{G}}_{2}$ be two SIVPFGs. If $\tilde{\mu}_{\tilde{\mathcal{P}}_{1}}^{L} \geq$ $\tilde{\mu}_{\tilde{\mathcal{Q}}_{2}}^{L}, \tilde{v}_{\tilde{\mathcal{P}}_{1}}^{U} \leq \tilde{v}_{\tilde{\mathcal{Q}}_{2}}^{U}, \tilde{\mu}_{\tilde{\mathcal{P}}_{1}}^{U} \geq \tilde{\mu}_{\tilde{\mathcal{Q}}_{2}}^{U}, \tilde{v}_{\tilde{\mathcal{P}}_{1}}^{L} \leq \tilde{v}_{\tilde{\mathcal{Q}}_{2}}^{L}$ and $\tilde{\mu}_{\tilde{\mathcal{P}}_{2}}^{L} \geq$ $\tilde{\mu}_{\tilde{\mathcal{Q}}_{1}}^{L}, \tilde{v}_{\tilde{\mathcal{P}}_{2}}^{U} \leq \tilde{v}_{\tilde{\mathcal{Q}}_{1}}^{U}, \tilde{\mu}_{\tilde{\mathcal{P}}_{2}}^{U} \geq \tilde{\mu}_{\tilde{\mathcal{Q}}_{1}}^{U}, \tilde{v}_{\tilde{\mathcal{P}}_{2}}^{L} \leq \tilde{v}_{\tilde{\mathcal{Q}}_{1}}^{L}$. Then $\mathrm{d}_{\tilde{\mathcal{G}}_{1}} \square \tilde{\mathcal{G}}_{2}\left(z_{1}, z_{2}\right)=\mathrm{d}_{\tilde{\mathcal{G}}_{1}}\left(z_{1}\right)+\mathrm{d}_{\tilde{\mathcal{G}}_{2}}\left(z_{2}\right)$ for all $\left(z_{1}, z_{2}\right) \in$ $V_{1} \times V_{2}$.

Proof By definition of vertex degree of $\tilde{\mathcal{G}}_{1} \square \tilde{\mathcal{G}}_{2}$, we have

$$
\begin{aligned}
& \left(\mathrm{d}_{\tilde{\mu}^{L}}\right)_{\tilde{\mathcal{G}}_{1}} \square \tilde{\mathcal{G}}_{2}\left(z_{1}, z_{2}\right) \\
& =\sum_{\left(y_{1}, y_{2}\right)\left(z_{1}, z_{2}\right) \in E_{1}} \square E_{2}\left(\tilde{\mu}_{\tilde{\mathcal{Q}}_{1}}^{L} \square \tilde{\mu}_{\tilde{\mathcal{Q}}_{2}}^{L}\right)\left(\left(y_{1}, y_{2}\right)\left(z_{1}, z_{2}\right)\right) \\
& =\sum_{y_{1}=z_{1}, y_{2} z_{2} \in E_{2}} \tilde{\mu}_{\tilde{\mathcal{P}}_{1}}^{L}\left(z_{1}\right) \wedge \tilde{\mu}_{\tilde{\mathcal{Q}}_{2}}^{L}\left(y_{2} z_{2}\right) \\
& +\sum_{y_{2}=z_{2}, y_{1} z_{1} \in E_{1}} \tilde{\mu}_{\tilde{\mathcal{P}}_{2}}^{L}\left(z_{2}\right) \wedge \tilde{\mu}_{\tilde{\mathcal{Q}}_{1}}^{L}\left(y_{1} z_{1}\right) \\
& =\sum_{y_{2} z_{2} \in E_{2}} \tilde{\mu}_{\tilde{\mathcal{Q}}_{2}}^{L}\left(y_{2} z_{2}\right)+\sum_{y_{1} z_{1} \in E_{1}} \tilde{\mu}_{\tilde{\mathcal{Q}}_{1}}^{L}\left(y_{1} z_{1}\right) \\
& \text { (using } \left.\tilde{\mu}_{\tilde{\mathcal{P}}_{1}}^{L} \geq \tilde{\mu}_{\tilde{\mathcal{Q}}_{2}}^{L} \text { and } \tilde{\mu}_{\tilde{\mathcal{P}}_{2}}^{L} \geq \tilde{\mu}_{\tilde{\mathcal{Q}}_{1}}^{L}\right) \\
& =\left(\mathrm{d}_{\tilde{\mu}^{L}}\right)_{\tilde{\mathcal{G}}_{1}}\left(z_{1}\right)+\left(\mathrm{d}_{\tilde{\mu}^{L}}\right)_{\tilde{\mathcal{G}}_{2}}\left(z_{2}\right), \\
& \left(\mathrm{d}_{\tilde{\nu} U}\right)_{\tilde{\mathcal{G}}_{1}} \square \tilde{\mathcal{G}}_{2}\left(z_{1}, z_{2}\right) \\
& =\sum_{\left(y_{1}, y_{2}\right)\left(z_{1}, z_{2}\right) \in E_{1} \square E_{2}}\left(\tilde{v}_{\tilde{\mathcal{Q}}_{1}}^{U} \square \tilde{v}_{\tilde{\mathcal{Q}}_{2}}^{U}\right)\left(\left(y_{1}, y_{2}\right)\left(z_{1}, z_{2}\right)\right)
\end{aligned}
$$




$$
\begin{aligned}
& =\sum_{y_{1}=z_{1}, y_{2} z_{2} \in E_{2}} \tilde{\mathcal{\nu}}_{\tilde{\mathcal{P}}_{1}}^{U}\left(z_{1}\right) \vee \tilde{v}_{\tilde{\mathcal{Q}}_{2}}^{U}\left(y_{2} z_{2}\right) \\
& +\sum_{y_{2}=z_{2}, y_{1} z_{1} \in E_{1}} \tilde{\nu}_{\tilde{\mathcal{P}}_{2}}^{U}\left(z_{2}\right) \vee \tilde{v}_{\tilde{\mathcal{Q}}_{1}}^{U}\left(y_{1} z_{1}\right) \\
& =\sum_{y_{2} z_{2} \in E_{2}} \tilde{v}_{\tilde{\mathcal{Q}}_{2}}^{U}\left(y_{2} z_{2}\right)+\sum_{y_{1} z_{1} \in E_{1}} \tilde{\nu}_{\tilde{\mathcal{Q}}_{1}}^{U}\left(y_{1} z_{1}\right) \\
& \text { (using } \tilde{v}_{\tilde{\mathcal{P}}_{1}}^{U} \leq \tilde{v}_{\tilde{\mathcal{Q}}_{2}}^{U} \text { and } \tilde{v}_{\tilde{\mathcal{P}}_{2}}^{U} \leq \tilde{v}_{\tilde{\mathcal{Q}}_{1}}^{U} \text { ) } \\
& =\left(\mathrm{d}_{\tilde{v} U}\right)_{\tilde{\mathcal{G}}_{1}}\left(z_{1}\right)+\left(\mathrm{d}_{\tilde{v} U}\right)_{\tilde{\mathcal{G}}_{2}}\left(z_{2}\right), \\
& \left(\mathrm{d}_{\tilde{\mu}^{U}}\right)_{\tilde{\mathcal{G}}_{1}} \square \tilde{\mathcal{G}}_{2}\left(z_{1}, z_{2}\right) \\
& =\sum_{\left(y_{1}, y_{2}\right)\left(z_{1}, z_{2}\right) \in E_{1} \square E_{2}}\left(\tilde{\mu}_{\tilde{\mathcal{Q}}_{1}}^{U} \square \tilde{\mu}_{\tilde{\mathcal{Q}}_{2}}^{U}\right)\left(\left(y_{1}, y_{2}\right)\left(z_{1}, z_{2}\right)\right) \\
& =\sum_{y_{1}=z_{1}, y_{2} z_{2} \in E_{2}} \tilde{\mu}_{\tilde{\mathcal{P}}_{1}}^{U}\left(z_{1}\right) \wedge \tilde{\mu}_{\tilde{\mathcal{Q}}_{2}}^{U}\left(y_{2} z_{2}\right) \\
& +\sum_{y_{2}=z_{2}, y_{1} z_{1} \in E_{1}} \tilde{\mu}_{\tilde{\mathcal{P}}_{2}}^{U}\left(z_{2}\right) \wedge \tilde{\mu}_{\tilde{\mathcal{Q}}_{1}}^{U}\left(y_{1} z_{1}\right) \\
& =\sum_{y_{2} z_{2} \in E_{2}} \tilde{\mu}_{\tilde{\mathcal{Q}}_{2}}^{U}\left(y_{2} z_{2}\right)+\sum_{y_{1} z_{1} \in E_{1}} \tilde{\mu}_{\tilde{\mathcal{Q}}_{1}}^{U}\left(y_{1} z_{1}\right) \\
& \text { (using } \tilde{\mu}_{\tilde{\mathcal{P}}_{1}}^{U} \geq \tilde{\mu}_{\tilde{\mathcal{Q}}_{2}}^{U} \text { and } \tilde{\mu}_{\tilde{\mathcal{P}}_{2}}^{U} \geq \tilde{\mu}_{\tilde{\mathcal{Q}}_{1}}^{U} \text { ) } \\
& =\left(\mathrm{d}_{\tilde{\mu}^{U}}\right)_{\tilde{\mathcal{G}}_{1}}\left(z_{1}\right)+\left(\mathrm{d}_{\tilde{\mu}^{U}}\right)_{\tilde{\mathcal{G}}_{2}}\left(z_{2}\right), \\
& \left(\mathrm{d}_{\tilde{v}^{L}}\right)_{\tilde{\mathcal{G}}_{1}} \square \tilde{\mathcal{G}}_{2}\left(z_{1}, z_{2}\right) \\
& =\sum_{\left(y_{1}, y_{2}\right)\left(z_{1}, z_{2}\right) \in E_{1}}^{\square E_{2}}\left(\tilde{v}_{\tilde{\mathcal{Q}}_{1}}^{L} \square \tilde{v}_{\tilde{\mathcal{Q}}_{2}}^{L}\right)\left(\left(y_{1}, y_{2}\right)\left(z_{1}, z_{2}\right)\right) \\
& =\sum_{y_{1}=z_{1}, y_{2} z_{2} \in E_{2}} \tilde{v}_{\tilde{\mathcal{P}}_{1}}^{L}\left(z_{1}\right) \vee \tilde{v}_{\tilde{\mathcal{Q}}_{2}}^{L}\left(y_{2} z_{2}\right) \\
& +\sum_{y_{2}=z_{2}, y_{1} z_{1} \in E_{1}} \tilde{\mathcal{P}}_{\tilde{\mathcal{P}}_{2}}^{L}\left(z_{2}\right) \vee \tilde{v}_{\tilde{\mathcal{Q}}_{1}}^{L}\left(y_{1} z_{1}\right) \\
& =\sum_{y_{2} z_{2} \in E_{2}} \tilde{v}_{\tilde{\mathcal{Q}}_{2}}^{L}\left(y_{2} z_{2}\right)+\sum_{y_{1} z_{1} \in E_{1}} \tilde{v}_{\tilde{\mathcal{Q}}_{1}}^{L}\left(y_{1} z_{1}\right) \\
& \text { (using } \left.\tilde{v}_{\tilde{\mathcal{P}}_{1}}^{L} \leq \tilde{v}_{\tilde{\mathcal{Q}}_{2}}^{L} \text { and } \tilde{v}_{\tilde{\mathcal{P}}_{2}}^{L} \leq \tilde{v}_{\tilde{\mathcal{Q}}_{1}}^{L}\right) \\
& =\left(\mathrm{d}_{\tilde{v}^{L}}\right)_{\tilde{\mathcal{G}}_{1}}\left(z_{1}\right)+\left(\mathrm{d}_{\tilde{v}^{L}}\right)_{\tilde{\mathcal{G}}_{2}}\left(z_{2}\right) .
\end{aligned}
$$

Hence $\mathrm{d}_{\tilde{\mathcal{G}}_{1}} \square \tilde{\mathcal{G}}_{2}\left(z_{1}, z_{2}\right)=\mathrm{d}_{\tilde{\mathcal{G}}_{1}}\left(z_{1}\right)+\mathrm{d}_{\tilde{\mathcal{G}}_{2}}\left(z_{2}\right)$.

Theorem 5 Let $\tilde{\mathcal{G}}_{1}$ and $\tilde{\mathcal{G}}_{2}$ be two SIVPFGs. If

(i) $\tilde{\mu}_{\tilde{\mathcal{P}}_{1}}^{L} \geq \tilde{\mu}_{\tilde{\mathcal{Q}}_{2}}^{L}$ and $\tilde{\mu}_{\tilde{\mathcal{P}}_{2}}^{L} \geq \tilde{\mu}_{\tilde{\mathcal{Q}}_{1}}^{L}$, then $\left(t \mathrm{~d}_{\tilde{\mu}^{L}}\right)_{\tilde{\mathcal{G}}_{1}} \square \tilde{\mathcal{G}}_{2}\left(z_{1}, z_{2}\right)$ $=\left(t \mathrm{~d}_{\tilde{\mu}^{L}}\right)_{\tilde{\mathcal{G}}_{1}}\left(z_{1}\right)+\left(t \mathrm{~d}_{\tilde{\mu}^{L}}\right)_{\tilde{\mathcal{G}}_{2}}\left(z_{2}\right)-\tilde{\mu}_{\tilde{\mathcal{P}}_{1}}^{L}\left(z_{1}\right) \vee \tilde{\mu}_{\tilde{\mathcal{P}}_{2}}^{L}\left(z_{2}\right) ;$

(ii) $\tilde{v}_{\tilde{\mathcal{P}}_{1}}^{U} \leq \tilde{v}_{\tilde{\mathcal{Q}}_{2}}^{U}$ and $\tilde{v}_{\tilde{\mathcal{P}}_{2}}^{U} \leq \tilde{v}_{\tilde{\mathcal{Q}}_{1}}^{U}$, then $\left(t \mathrm{~d}_{\tilde{v}^{U}}\right)_{\tilde{\mathcal{G}}_{1}} \square \tilde{\mathcal{G}}_{2}\left(z_{1}, z_{2}\right)$ $=\left(t \mathrm{~d}_{\tilde{v} U}\right)_{\tilde{\mathcal{G}}_{1}}\left(z_{1}\right)+\left(t \mathrm{~d}_{\tilde{v}^{U}}\right)_{\tilde{\mathcal{G}}_{2}}\left(z_{2}\right)-\tilde{v}_{\tilde{\mathcal{P}}_{1}}^{U}\left(z_{1}\right) \wedge \tilde{v}_{\tilde{\mathcal{P}}_{2}}^{U}\left(z_{2}\right) ;$

(iii) $\tilde{\mu}_{\tilde{\mathcal{P}}_{1}}^{U} \geq \tilde{\mu}_{\tilde{\mathcal{Q}}_{2}}^{U}$ and $\tilde{\mu}_{\tilde{\mathcal{P}}_{2}}^{U} \geq \tilde{\mu}_{\tilde{\mathcal{Q}}_{1}}^{U}$, then $\left(t \mathrm{~d}_{\tilde{\mu}^{U}}\right)_{\tilde{\mathcal{G}}_{1}} \square \tilde{\mathcal{G}}_{2}\left(z_{1}, z_{2}\right)$ $=\left(t \mathrm{~d}_{\tilde{\mu}^{U}}\right)_{\tilde{\mathcal{G}}_{1}}\left(z_{1}\right)+\left(t \mathrm{~d}_{\tilde{\mu}^{U}}\right) \tilde{\mathcal{G}}_{2}\left(z_{2}\right)-\tilde{\mu}_{\tilde{\mathcal{P}}_{1}}^{U}\left(z_{1}\right) \vee \tilde{\mu}_{\tilde{\mathcal{P}}_{2}}^{U}\left(z_{2}\right) ;$

(iv) $\tilde{v}_{\tilde{\mathcal{P}}_{1}}^{L} \leq \tilde{v}_{\tilde{\mathcal{Q}}_{2}}^{L}$ and $\tilde{v}_{\tilde{\mathcal{P}}_{2}}^{L} \leq \tilde{v}_{\tilde{\mathcal{Q}}_{1}}^{L}$, then $\left(t \mathrm{~d}_{\tilde{v}^{L}}\right)_{\tilde{\mathcal{G}}_{1}} \square \tilde{\mathcal{G}}_{2}\left(z_{1}, z_{2}\right)$ $=\left(t \mathrm{~d}_{\tilde{v}^{L}}\right)_{\tilde{\mathcal{G}}_{1}}\left(z_{1}\right)+\left(t \mathrm{~d}_{\tilde{v}^{L}}\right)_{\tilde{\mathcal{G}}_{2}}\left(z_{2}\right)-\tilde{v}_{\tilde{\mathcal{P}}_{1}}^{L}\left(z_{1}\right) \wedge \tilde{v}_{\tilde{\mathcal{P}}_{2}}^{L}\left(z_{2}\right)$ for all $\left(z_{1}, z_{2}\right) \in V_{1} \times V_{2}$.

Proof Utilizing the definition of vertex total degree of $\tilde{\mathcal{G}}_{1} \square \tilde{\mathcal{G}}_{2}$,

(i) If $\tilde{\mu}_{\tilde{\mathcal{P}}_{1}}^{L} \geq \tilde{\mu}_{\tilde{\mathcal{Q}}_{2}}^{L}, \tilde{\mu}_{\tilde{\mathcal{P}}_{2}}^{L} \geq \tilde{\mu}_{\tilde{\mathcal{Q}}_{1}}^{L}$

$$
\begin{aligned}
(t & \left.\mathrm{d}_{\tilde{\mu}^{L}}\right)_{\tilde{\mathcal{G}}_{1}} \square \tilde{\mathcal{G}}_{2}\left(z_{1}, z_{2}\right) \\
= & \sum_{\left(y_{1}, y_{2}\right)\left(z_{1}, z_{2}\right) \in E_{1}}\left(\tilde{\mu}_{2} E_{\tilde{\mathcal{Q}}_{1}}^{L} \square \tilde{\mu}_{\tilde{\mathcal{Q}}_{2}}^{L}\right)\left(\left(y_{1}, y_{2}\right)\left(z_{1}, z_{2}\right)\right) \\
& +\left(\tilde{\mu}_{\tilde{\mathcal{P}}_{1}}^{L} \square \tilde{\mu}_{\tilde{\mathcal{P}}_{2}}^{L}\right)\left(z_{1}, z_{2}\right) \\
= & \sum_{y_{1}=z_{1}, y_{2} z_{2} \in E_{2}} \tilde{\mu}_{\tilde{\mathcal{P}}_{1}}^{L}\left(z_{1}\right) \wedge \tilde{\mu}_{\tilde{\mathcal{Q}}_{2}}^{L}\left(y_{2} z_{2}\right) \\
& +\sum_{y_{2}=z_{2}, y_{1} z_{1} \in E_{1}} \tilde{\mu}_{\tilde{\mathcal{P}}_{2}}^{L}\left(z_{2}\right) \wedge \tilde{\mu}_{\tilde{\mathcal{Q}}_{1}}^{L}\left(y_{1} z_{1}\right) \\
& +\tilde{\mu}_{\tilde{\mathcal{P}}_{1}}^{L}\left(z_{1}\right) \wedge \tilde{\mu}_{\tilde{\mathcal{P}}_{2}}^{L}\left(z_{2}\right) \\
= & \sum_{y_{2} z_{2} \in E_{2}} \tilde{\mu}_{\tilde{\mathcal{Q}}_{2}}^{L}\left(y_{2} z_{2}\right)+\sum_{y_{1} z_{1} \in E_{1}} \tilde{\mu}_{\tilde{\mathcal{Q}}_{1}}^{L}\left(y_{1} z_{1}\right) \\
& +\tilde{\mu}_{\tilde{\mathcal{P}}_{1}}^{L}\left(z_{1}\right)+\tilde{\mu}_{\tilde{\mathcal{P}}_{2}}^{L}\left(z_{2}\right)-\tilde{\mu}_{\tilde{\mathcal{P}}_{1}}^{L}\left(z_{1}\right) \vee \tilde{\mu}_{\tilde{\mathcal{P}}_{2}}^{L}\left(z_{2}\right) \\
= & \sum_{y_{2} z_{2} \in E_{2}} \tilde{\mu}_{\tilde{\mathcal{Q}}_{2}}^{L}\left(y_{2} z_{2}\right)+\tilde{\mu}_{\tilde{\mathcal{P}}_{2}}^{L}\left(z_{2}\right)+\sum_{y_{1} z_{1} \in E_{1}} \tilde{\mu}_{\tilde{\mathcal{Q}}_{1}}^{L}\left(y_{1} z_{1}\right) \\
& +\tilde{\mu}_{\tilde{\mathcal{P}}_{1}}^{L}\left(z_{1}\right)-\tilde{\mu}_{\tilde{\mathcal{P}}_{1}}^{L}\left(z_{1}\right) \vee \tilde{\mu}_{\tilde{\mathcal{P}}_{2}}^{L}\left(z_{2}\right) \\
=\left(t \mathrm{~d}_{\tilde{\mu}^{L}}\right)_{\tilde{\mathcal{G}}_{1}}\left(z_{1}\right)+\left(t \mathrm{~d}_{\tilde{\mu}^{L}}\right) & \tilde{\mathcal{G}}_{2}\left(z_{2}\right)-\tilde{\mu}_{\tilde{\mathcal{P}}_{1}}^{L}\left(z_{1}\right) \vee \tilde{\mu}_{\tilde{\mathcal{P}}_{2}}^{L}\left(z_{2}\right), \\
&
\end{aligned}
$$

Analogously, we can prove (ii)-(iv).

Definition 17 Let $\tilde{\mathcal{G}}_{1}$ and $\tilde{\mathcal{G}}_{2}$ be two SIVPFGs of the graphs $G_{1}$ and $G_{2}$, respectively. The semi-strong product of $\tilde{\mathcal{G}}_{1}$ and $\tilde{\mathcal{G}}_{2}$, denoted by $\tilde{\mathcal{G}}_{1} \bullet \tilde{\mathcal{G}}_{2}=\left(\tilde{\mathcal{P}}_{1} \bullet \tilde{\mathcal{P}}_{2}, \tilde{\mathcal{Q}}_{1} \bullet \tilde{\mathcal{Q}}_{2}\right)$, is defined as:

(i)

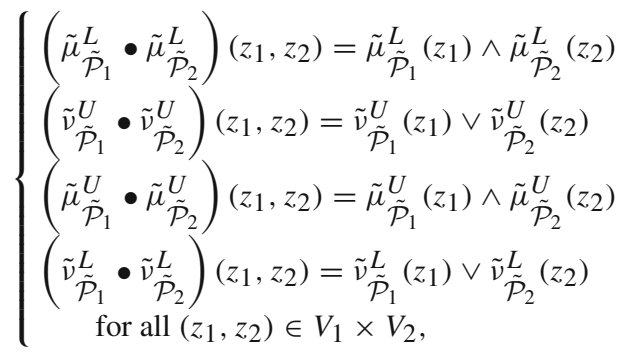

(ii)

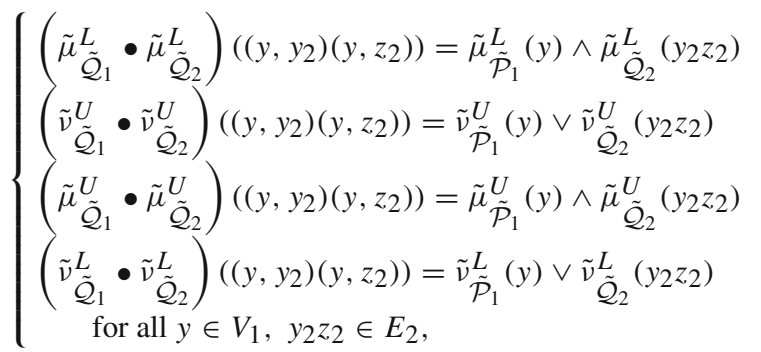




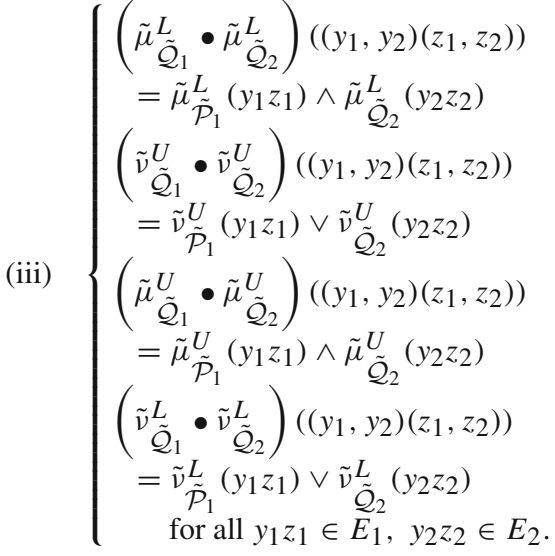

Proposition 3 The semi-strong product $\tilde{\mathcal{G}}_{1}$ • $\tilde{\mathcal{G}}_{2}$ of two SIVPFGs $\tilde{\mathcal{G}}_{1}$ and $\tilde{\mathcal{G}}_{2}$ is a SIVPFG.

Definition 18 Let $\tilde{\mathcal{G}}_{1}$ and $\tilde{\mathcal{G}}_{2}$ be two SIVPFGs. For any vertex $\left(z_{1}, z_{2}\right) \in V_{1} \times V_{2}$,

$$
\begin{aligned}
& \left(\mathrm{d}_{\tilde{\mu}^{L}}\right)_{\tilde{\mathcal{G}}_{1} \bullet \tilde{\mathcal{G}}_{2}}\left(z_{1}, z_{2}\right) \\
& =\sum_{\left(y_{1}, y_{2}\right)\left(z_{1}, z_{2}\right) \in E_{1} \bullet E_{2}}\left(\tilde{\mu}_{\tilde{\mathcal{Q}}_{1}}^{L} \bullet \tilde{\mu}_{\tilde{\mathcal{Q}}_{2}}^{L}\right)\left(\left(y_{1}, y_{2}\right)\left(z_{1}, z_{2}\right)\right) \\
& =\sum_{y_{1}=z_{1}, y_{2} z_{2} \in E_{2}} \tilde{\mu}_{\tilde{\mathcal{P}}_{1}}^{L}\left(z_{1}\right) \wedge \tilde{\mu}_{\tilde{\mathcal{Q}}_{2}}^{L}\left(y_{2} z_{2}\right) \\
& +\sum_{y_{1} z_{1} \in E_{1}, y_{2} z_{2} \in E_{2}} \tilde{\mu}_{\tilde{\mathcal{Q}}_{1}}^{L}\left(y_{1} z_{1}\right) \wedge \tilde{\mu}_{\tilde{\mathcal{Q}}_{2}}^{L}\left(y_{2} z_{2}\right), \\
& \left(\mathrm{d}_{\tilde{\nu}^{U}}\right)_{\tilde{\mathcal{G}}_{1}} \bullet \tilde{\mathcal{G}}_{2}\left(z_{1}, z_{2}\right) \\
& =\sum_{\left(y_{1}, y_{2}\right)\left(z_{1}, z_{2}\right) \in E_{1} \bullet E_{2}}\left(\tilde{v}_{\tilde{\mathcal{Q}}_{1}}^{U} \bullet \tilde{v}_{\tilde{\mathcal{Q}}_{2}}^{U}\right)\left(\left(y_{1}, y_{2}\right)\left(z_{1}, z_{2}\right)\right) \\
& =\sum_{y_{1}=z_{1}, y_{2} z_{2} \in E_{2}} \tilde{\nu}_{\tilde{\mathcal{P}}_{1}}^{U}\left(z_{1}\right) \vee \tilde{\nu}_{\tilde{\mathcal{Q}}_{2}}^{U}\left(y_{2} z_{2}\right) \\
& +\sum_{y_{1} z_{1} \in E_{1}, y_{2} z_{2} \in E_{2}} \tilde{v}_{\tilde{\mathcal{Q}}_{1}}^{U}\left(y_{1} z_{1}\right) \vee \tilde{v}_{\tilde{\mathcal{Q}}_{2}}^{U}\left(y_{2} z_{2}\right), \\
& \left(\mathrm{d}_{\tilde{\mu}^{U}}\right)_{\tilde{\mathcal{G}}_{1}} \bullet \tilde{\mathcal{G}}_{2}\left(z_{1}, z_{2}\right) \\
& =\sum_{\left(y_{1}, y_{2}\right)\left(z_{1}, z_{2}\right) \in E_{1} \bullet E_{2}}\left(\tilde{\mu}_{\tilde{\mathcal{Q}}_{1}}^{U} \bullet \tilde{\mu}_{\tilde{\mathcal{Q}}_{2}}^{U}\right)\left(\left(y_{1}, y_{2}\right)\left(z_{1}, z_{2}\right)\right) \\
& =\sum_{y_{1}=z_{1}, y_{2} z_{2} \in E_{2}} \tilde{\mu}_{\tilde{\mathcal{P}}_{1}}^{U}\left(z_{1}\right) \wedge \tilde{\mu}_{\tilde{\mathcal{Q}}_{2}}^{U}\left(y_{2} z_{2}\right) \\
& +\sum_{y_{1} z_{1} \in E_{1}, y_{2} z_{2} \in E_{2}} \tilde{\mu}_{\tilde{\mathcal{Q}}_{1}}^{U}\left(y_{1} z_{1}\right) \wedge \tilde{\mu}_{\tilde{\mathcal{Q}}_{2}}^{U}\left(y_{2} z_{2}\right), \\
& \left(\mathrm{d}_{\tilde{v}^{L}}\right)_{\tilde{\mathcal{G}}_{1} \bullet \tilde{\mathcal{G}}_{2}}\left(z_{1}, z_{2}\right) \\
& =\sum_{\left(y_{1}, y_{2}\right)\left(z_{1}, z_{2}\right) \in E_{1} \bullet E_{2}}\left(\tilde{v}_{\tilde{\mathcal{Q}}_{1}}^{L} \bullet \tilde{v}_{\tilde{\mathcal{Q}}_{2}}^{L}\right)\left(\left(y_{1}, y_{2}\right)\left(z_{1}, z_{2}\right)\right) \\
& =\sum_{y_{1}=z_{1}, y_{2} z_{2} \in E_{2}} \tilde{\mathcal{\nu}}_{\tilde{\mathcal{P}}_{1}}^{L}\left(z_{1}\right) \vee \tilde{v}_{\tilde{\mathcal{Q}}_{2}}^{L}\left(y_{2} z_{2}\right) \\
& +\sum_{y_{1} z_{1} \in E_{1}, y_{2} z_{2} \in E_{2}} \tilde{\nu}_{\tilde{\mathcal{Q}}_{1}}^{L}\left(y_{1} z_{1}\right) \vee \tilde{v}_{\tilde{\mathcal{Q}}_{2}}^{L}\left(y_{2} z_{2}\right) .
\end{aligned}
$$

Theorem 6 Let $\tilde{\mathcal{G}}_{1}$ and $\tilde{\mathcal{G}}_{2}$ be two SIVPFGs. If $\tilde{\mu}_{\tilde{\mathcal{P}}_{1}}^{L} \geq$ $\tilde{\mu}_{\tilde{\mathcal{Q}}_{2}}^{L}, \tilde{v}_{\tilde{\mathcal{P}}_{1}}^{U} \leq \tilde{v}_{\tilde{\mathcal{Q}}_{2}}^{U}, \tilde{\mu}_{\tilde{\mathcal{P}}_{1}}^{U} \geq \tilde{\mu}_{\tilde{\mathcal{Q}}_{2}}^{U}, \tilde{v}_{\tilde{\mathcal{P}}_{1}}^{L} \leq \tilde{v}_{\tilde{\mathcal{Q}}_{2}}^{L}, \tilde{\mu}_{\tilde{\mathcal{Q}}_{1}}^{L} \leq$ $\tilde{\mu}_{\tilde{\mathcal{Q}}_{2}}^{L}, \tilde{v}_{\tilde{\mathcal{Q}}_{1}}^{U} \geq \tilde{v}_{\tilde{\mathcal{Q}}_{2}}^{U}, \tilde{\mu}_{\tilde{\mathcal{Q}}_{1}}^{U} \leq \tilde{\mu}_{\tilde{\mathcal{Q}}_{2}}^{U}, \tilde{v}_{\tilde{\mathcal{Q}}_{1}}^{L} \geq \tilde{v}_{\tilde{\mathcal{Q}}_{2}}^{L} \cdot$ Then $\mathrm{d}_{\tilde{\mathcal{G}}_{1} \bullet \tilde{\mathcal{G}}_{2}}\left(z_{1}, z_{2}\right)=\mathrm{d}_{\tilde{\mathcal{G}}_{1}}\left(z_{1}\right)+\mathrm{d}_{\tilde{\mathcal{G}}_{2}}\left(z_{2}\right)$ for all $\left(z_{1}, z_{2}\right) \in V_{1} \times V_{2}$.

Proof The proof follows at once from the proof of Theorems 2 and 4.

Theorem 7 Let $\tilde{\mathcal{G}}_{1}$ and $\tilde{\mathcal{G}}_{2}$ be two SIVPFGs. If

(i) $\tilde{\mu}_{\tilde{\mathcal{P}}_{1}}^{L} \geq \tilde{\mu}_{\tilde{\mathcal{Q}}_{2}}^{L}, \tilde{\mu}_{\tilde{\mathcal{Q}}_{1}}^{L} \leq \tilde{\mu}_{\tilde{\mathcal{Q}}_{2}}^{L}$, then $\left(t \mathrm{~d}_{\tilde{\mu}^{L}}\right)_{\tilde{\mathcal{G}}_{1}} \bullet \tilde{\mathcal{G}}_{2}\left(z_{1}, z_{2}\right)=$ $\left(t \mathrm{~d}_{\tilde{\mu}^{L}}\right)_{\tilde{\mathcal{G}}_{1}}\left(z_{1}\right)+\left(t \mathrm{~d}_{\tilde{\mu}^{L}}\right)_{\tilde{\mathcal{G}}_{2}}\left(z_{2}\right)-\tilde{\mu}_{\tilde{\mathcal{P}}_{1}}^{L}\left(z_{1}\right) \vee \tilde{\mu}_{\tilde{\mathcal{P}}_{2}}^{L}\left(z_{2}\right) ;$

(ii) $\tilde{v}_{\tilde{\mathcal{P}}_{1}}^{U} \leq \tilde{v}_{\tilde{\mathcal{Q}}_{2}}^{U}, \tilde{v}_{\tilde{\mathcal{Q}}_{1}}^{U} \geq \tilde{v}_{\tilde{\mathcal{Q}}_{2}}^{U}$, then $\left(t \mathrm{~d}_{\tilde{v} U}\right)_{\tilde{\mathcal{G}}_{1} \bullet \tilde{\mathcal{G}}_{2}}\left(z_{1}, z_{2}\right)=$ $\left(t \mathrm{~d}_{\tilde{v} U}\right)_{\tilde{\mathcal{G}}_{1}}\left(z_{1}\right)+\left(t \mathrm{~d}_{\tilde{v} U}\right)_{\tilde{\mathcal{G}}_{2}}\left(z_{2}\right)-\tilde{v}_{\tilde{\mathcal{P}}_{1}}^{U}\left(z_{1}\right) \wedge \tilde{v}_{\tilde{\mathcal{P}}_{2}}^{U}\left(z_{2}\right) ;$

(iii) $\tilde{\mu}_{\tilde{\mathcal{P}}_{1}}^{U} \geq \tilde{\mu}_{\tilde{\mathcal{Q}}_{2}}^{U}, \tilde{\mu}_{\tilde{\mathcal{Q}}_{1}}^{U} \leq \tilde{\mu}_{\tilde{\mathcal{Q}}_{2}}^{U}$, then $\left(t \mathrm{~d}_{\tilde{\mu}^{U}}\right)_{\tilde{\mathcal{G}}_{1} \bullet \tilde{\mathcal{G}}_{2}}\left(z_{1}, z_{2}\right)=$ $\left(t \mathrm{~d}_{\tilde{\mu}^{U}}\right)_{\tilde{\mathcal{G}}_{1}}\left(z_{1}\right)+\left(t \mathrm{~d}_{\tilde{\mu}^{U}}\right)_{\tilde{\mathcal{G}}_{2}}\left(z_{2}\right)-\tilde{\mu}_{\tilde{\mathcal{P}}_{1}}^{U}\left(z_{1}\right) \vee \tilde{\mu}_{\tilde{\mathcal{P}}_{2}}^{U}\left(z_{2}\right) ;$

(iv) $\tilde{v}_{\tilde{\mathcal{P}}_{1}}^{L} \leq \tilde{v}_{\tilde{\mathcal{Q}}_{2}}^{L}, \tilde{v}_{\tilde{\mathcal{Q}}_{1}}^{L} \geq \tilde{v}_{\tilde{\mathcal{Q}}_{2}}^{L}$, then $\left(t \mathrm{~d}_{\tilde{v}^{L}}\right)_{\tilde{\mathcal{G}}_{1} \bullet \tilde{\mathcal{G}}_{2}}\left(z_{1}, z_{2}\right)=$ $\left(t \mathrm{~d}_{\tilde{v}^{L}}\right)_{\tilde{\mathcal{G}}_{1}}\left(z_{1}\right)+\left(t \mathrm{~d}_{\left.\tilde{v}^{L}\right)}\right)_{\tilde{\mathcal{G}}_{2}}\left(z_{2}\right)-\tilde{v}_{\tilde{\mathcal{P}}_{1}}^{L}\left(z_{1}\right) \wedge \tilde{v}_{\tilde{\mathcal{P}}_{2}}^{L}\left(z_{2}\right)$

for all $\left(z_{1}, z_{2}\right) \in V_{1} \times V_{2}$.

Proof The proof follows at once from the proof of Theorems 3 and 5.

Definition 19 Let $\tilde{\mathcal{G}}_{1}$ and $\tilde{\mathcal{G}}_{2}$ be two SIVPFGs of $G_{1}$ and $G_{2}$, respectively. The strong product of these two SIVPFGs is denoted by $\tilde{\mathcal{G}}_{1} \nabla \tilde{\mathcal{G}}_{2}=\left(\tilde{\mathcal{P}}_{1} \otimes \tilde{\mathcal{P}}_{2}, \tilde{\mathcal{Q}}_{1} \otimes \tilde{\mathcal{Q}}_{2}\right)$ and defined as:

(i)

$$
\begin{aligned}
& \left\{\begin{array}{l}
\left(\tilde{\mu}_{\tilde{\mathcal{P}}_{1}}^{L} \otimes \tilde{\mu}_{\tilde{\mathcal{P}}_{2}}^{L}\right)\left(z_{1}, z_{2}\right)=\tilde{\mu}_{\tilde{\mathcal{P}}_{1}}^{L}\left(z_{1}\right) \wedge \tilde{\mu}_{\tilde{\mathcal{P}}_{2}}^{L}\left(z_{2}\right) \\
\left(\tilde{v}_{\tilde{\mathcal{P}}_{1}}^{U} \otimes \tilde{v}_{\tilde{\mathcal{P}}_{2}}^{U}\right)\left(z_{1}, z_{2}\right)=\tilde{v}_{\tilde{\mathcal{P}}_{1}}^{U}\left(z_{1}\right) \vee \tilde{v}_{\tilde{\mathcal{P}}_{2}}^{U}\left(z_{2}\right) \\
\left(\tilde{\mu}_{\tilde{\mathcal{P}}_{1}}^{U} \otimes \tilde{\mu}_{\tilde{\mathcal{P}}_{2}}^{U}\right)\left(z_{1}, z_{2}\right)=\tilde{\mu}_{\tilde{\mathcal{P}}_{1}}^{U}\left(z_{1}\right) \wedge \tilde{\mu}_{\tilde{\mathcal{P}}_{2}}^{U}\left(z_{2}\right) \\
\left(\tilde{v}_{\tilde{\mathcal{P}}_{1}}^{L} \otimes \tilde{v}_{\tilde{\mathcal{P}}_{2}}^{L}\right)\left(z_{1}, z_{2}\right)=\tilde{v}_{\tilde{\mathcal{P}}_{1}}^{L}\left(z_{1}\right) \vee \tilde{v}_{\tilde{\mathcal{P}}_{2}}^{L}\left(z_{2}\right) \\
\quad \text { for all }\left(z_{1}, z_{2}\right) \in V_{1} \times V_{2},
\end{array}\right. \\
& \int\left(\tilde{\mu}_{\tilde{\mathcal{Q}}_{1}}^{L} \otimes \tilde{\mu}_{\tilde{\mathcal{Q}}_{2}}^{L}\right)\left(\left(y, y_{2}\right)\left(y, z_{2}\right)\right) \\
& =\tilde{\mu}_{\tilde{\mathcal{P}}_{1}}^{L}(y) \wedge \tilde{\mu}_{\tilde{\mathcal{Q}}_{2}}^{L}\left(y_{2} z_{2}\right) \\
& \left(\tilde{v}_{\tilde{\mathcal{Q}}_{1}}^{U} \otimes \tilde{\nu}_{\tilde{\mathcal{Q}}_{2}}^{U}\right)\left(\left(y, y_{2}\right)\left(y, z_{2}\right)\right) \\
& =\tilde{\nu}_{\tilde{\mathcal{P}}_{1}}^{U}(y) \vee \tilde{\nu}_{\tilde{\mathcal{Q}}_{2}}^{U}\left(y_{2} z_{2}\right) \\
& \left(\tilde{\mu}_{\tilde{\mathcal{Q}}_{1}}^{U} \otimes \tilde{\mu}_{\tilde{\mathcal{Q}}_{2}}^{U}\right)\left(\left(y, y_{2}\right)\left(y, z_{2}\right)\right) \\
& =\tilde{\mu}_{\tilde{\mathcal{P}}_{1}}^{U}(y) \wedge \tilde{\mu}_{\tilde{\mathcal{Q}}_{2}}^{U}\left(y_{2} z_{2}\right) \\
& \left(\tilde{v}_{\tilde{\mathcal{Q}}_{1}}^{L} \otimes \tilde{v}_{\tilde{\mathcal{Q}}_{2}}^{L}\right)\left(\left(y, y_{2}\right)\left(y, z_{2}\right)\right) \\
& =\tilde{v}_{\tilde{\mathcal{P}}_{1}}^{L}(y) \vee \tilde{v}_{\tilde{\mathcal{Q}}_{2}}^{L}\left(y_{2} z_{2}\right) \\
& \text { for all } y \in V_{1} \text {, for all } y_{2} z_{2} \in E_{2} \text {, }
\end{aligned}
$$


(iii)

$\left\{\begin{array}{l}\left(\tilde{\mu}_{\tilde{\mathcal{Q}}_{1}}^{L} \otimes \tilde{\mu}_{\tilde{\mathcal{Q}}_{2}}^{L}\right)\left(\left(y_{1}, z\right)\left(z_{1}, z\right)\right) \\ =\tilde{\mu}_{\tilde{\mathcal{Q}}_{1}}^{L}\left(y_{1} z_{1}\right) \wedge \tilde{\mu}_{\tilde{\mathcal{P}}_{2}}^{L}(z) \\ \left(\tilde{v}_{\tilde{\mathcal{Q}}_{1}}^{U} \otimes \tilde{v}_{\tilde{\mathcal{Q}}_{2}}^{U}\right)\left(\left(y_{1}, z\right)\left(z_{1}, z\right)\right) \\ =\tilde{v}_{\tilde{\mathcal{Q}}_{1}}^{U}\left(y_{1} z_{1}\right) \vee \tilde{v}_{\tilde{\mathcal{P}}_{2}}^{U}(z) \\ \left(\tilde{\mu}_{\tilde{\mathcal{Q}}_{1}}^{U} \otimes \tilde{\mu}_{\tilde{\mathcal{Q}}_{2}}^{U}\right)\left(\left(y_{1}, z\right)\left(z_{1}, z\right)\right) \\ =\tilde{\mu}_{\tilde{\mathcal{Q}}_{1}}^{U}\left(y_{1} z_{1}\right) \wedge \tilde{\mu}_{\tilde{\mathcal{P}}_{2}}^{U}(z) \\ \left(\tilde{v}_{\tilde{\mathcal{Q}}_{1}}^{L} \otimes \tilde{v}_{\tilde{\mathcal{Q}}_{2}}^{L}\right)\left(\left(y_{1}, z\right)\left(z_{1}, z\right)\right) \\ =\tilde{v}_{\tilde{\mathcal{Q}}_{1}}^{L}\left(y_{1} z_{1}\right) \vee \tilde{v}_{\tilde{\mathcal{P}}_{2}}^{L}(z) \\ \quad \text { for all } z \in V_{2}, \text { for all } y_{1} z_{1} \in E_{1},\end{array}\right.$

(iv)

$$
\left\{\begin{array}{l}
\left(\tilde{\mu}_{\tilde{\mathcal{Q}}_{1}}^{L} \otimes \tilde{\mu}_{\tilde{\mathcal{Q}}_{2}}^{L}\right)\left(\left(y_{1}, y_{2}\right)\left(z_{1}, z_{2}\right)\right) \\
=\tilde{\mu}_{\tilde{\mathcal{Q}}_{1}}^{L}\left(y_{1} z_{1}\right) \wedge \tilde{\mu}_{\tilde{\mathcal{Q}}_{2}}^{L}\left(y_{2} z_{2}\right) \\
\left(\tilde{v}_{\tilde{\mathcal{Q}}_{1}}^{U} \otimes \tilde{v}_{\tilde{\mathcal{Q}}_{2}}^{U}\right)\left(\left(y_{1}, y_{2}\right)\left(z_{1}, z_{2}\right)\right) \\
=\tilde{v}_{\tilde{\mathcal{Q}}_{1}}^{U}\left(y_{1} z_{1}\right) \vee \tilde{v}_{\tilde{\mathcal{Q}}_{2}}^{U}\left(y_{2} z_{2}\right) \\
\left(\tilde{\mu}_{\tilde{\mathcal{Q}}_{1}}^{U} \otimes \tilde{\mu}_{\tilde{\mathcal{Q}}_{2}}^{U}\right)\left(\left(y_{1}, y_{2}\right)\left(z_{1}, z_{2}\right)\right) \\
=\tilde{\mu}_{\tilde{\mathcal{Q}}_{1}}^{U}\left(y_{1} z_{1}\right) \wedge \tilde{\mu}_{\tilde{\mathcal{Q}}_{2}}^{U}\left(y_{2} z_{2}\right) \\
\left(\tilde{v}_{\tilde{\mathcal{Q}}_{1}}^{L} \otimes \tilde{v}_{\tilde{\mathcal{Q}}_{2}}^{L}\right)\left(\left(y_{1}, y_{2}\right)\left(z_{1}, z_{2}\right)\right) \\
=\tilde{v}_{\tilde{\mathcal{Q}}_{1}}^{L}\left(y_{1} z_{1}\right) \vee \tilde{v}_{\tilde{\mathcal{Q}}_{2}}^{L}\left(y_{2} z_{2}\right) \\
\quad \text { for all } y_{1} z_{1} \in E_{1}, \text { for all } y_{2} z_{2} \in E_{2} .
\end{array}\right.
$$

Proposition 4 The strong product $\tilde{\mathcal{G}}_{1} \otimes \tilde{\mathcal{G}}_{2}$ of two SIVPFGs $\tilde{\mathcal{G}}_{1}$ and $\tilde{\mathcal{G}}_{2}$ is a SIVPFG.

Definition 20 Let $\tilde{\mathcal{G}}_{1}$ and $\tilde{\mathcal{G}}_{2}$ be two SIVPFGs. For any vertex $\left(z_{1}, z_{2}\right) \in V_{1} \times V_{2}$,

$$
\begin{aligned}
& \left(\mathrm{d}_{\tilde{\mu}^{L}}\right)_{\tilde{\mathcal{G}}_{1} \otimes \tilde{\mathcal{G}}_{2}}\left(z_{1}, z_{2}\right) \\
& =\sum_{\left(y_{1}, y_{2}\right)\left(z_{1}, z_{2}\right) \in E_{1} \otimes E_{2}}\left(\tilde{\mu}_{\tilde{\mathcal{Q}}_{1}}^{L} \otimes \tilde{\mu}_{\tilde{\mathcal{Q}}_{2}}^{L}\right)\left(\left(y_{1}, y_{2}\right)\left(z_{1}, z_{2}\right)\right) \\
& =\sum_{y_{1}=z_{1}, y_{2} z_{2} \in E_{2}} \tilde{\mu}_{\tilde{\mathcal{P}}_{1}}^{L}\left(z_{1}\right) \wedge \tilde{\mu}_{\tilde{\mathcal{Q}}_{2}}^{L}\left(y_{2} z_{2}\right) \\
& \quad+\sum_{y_{2}=z_{2}, y_{1} z_{1} \in E_{1}} \tilde{\mu}_{\tilde{\mathcal{P}}_{2}}^{L}\left(z_{2}\right) \wedge \tilde{\mu}_{\tilde{\mathcal{Q}}_{1}}^{L}\left(y_{1} z_{1}\right) \\
& \quad+\sum_{y_{1} z_{1} \in E_{1}, y_{2} z_{2} \in E_{2}} \tilde{\mu}_{\tilde{\mathcal{Q}}_{1}}^{L}\left(y_{1} z_{1}\right) \wedge \tilde{\mu}_{\tilde{\mathcal{Q}}_{2}}^{L}\left(y_{2} z_{2}\right), \\
& =\sum_{\tilde{\mathcal{G}}_{1} \otimes \tilde{\mathcal{G}}_{2}}\left(z_{1}, z_{2}\right) \\
& \quad \sum_{\left.y_{1}, y_{2}\right)\left(z_{1}, z_{2}\right) \in E_{1} \otimes E_{2}}\left(\tilde{v}_{y_{1}=z_{1}, y_{2} z_{2} \in E_{2}}^{U} \tilde{\mathcal{Q}}_{\tilde{\mathcal{P}}_{1}}^{U}\left(z_{1}\right) \vee \tilde{v}_{\tilde{\mathcal{Q}}_{2}}^{U}\right)\left(\left(y_{1}, y_{2}\right)\left(z_{1}, z_{2}\right)\right) \\
& \quad+\sum_{y_{\mathcal{Q}_{2}}=z_{2}, y_{1} z_{1} \in E_{1}} \tilde{v}_{\tilde{\mathcal{P}}_{2}}^{U}\left(y_{2} z_{2}\right) \vee \tilde{v}_{\tilde{\mathcal{Q}}_{1}}^{U}\left(y_{1} z_{1}\right) \\
& \quad+\sum_{y_{1} z_{1} \in E_{1}, y_{2} z_{2} \in E_{2}} \tilde{v}_{\tilde{\mathcal{Q}}_{1}}^{U}\left(y_{1} z_{1}\right) \vee \tilde{v}_{\tilde{\mathcal{Q}}_{2}}^{U}\left(y_{2} z_{2}\right),
\end{aligned}
$$

$$
\begin{aligned}
& \left(\mathrm{d}_{\tilde{\mu}^{U}}\right)_{\tilde{\mathcal{G}}_{1} \otimes \tilde{\mathcal{G}}_{2}}\left(z_{1}, z_{2}\right) \\
& =\sum_{\left(y_{1}, y_{2}\right)\left(z_{1}, z_{2}\right) \in E_{1} \otimes E_{2}}\left(\tilde{\mu}_{\tilde{\mathcal{Q}}_{1}}^{U} \otimes \tilde{\mu}_{\tilde{\mathcal{Q}}_{2}}^{U}\right)\left(\left(y_{1}, y_{2}\right)\left(z_{1}, z_{2}\right)\right) \\
& =\sum_{y_{1}=z_{1}, y_{2} z_{2} \in E_{2}} \tilde{\mu}_{\tilde{\mathcal{P}}_{1}}^{U}\left(z_{1}\right) \wedge \tilde{\mu}_{\tilde{\mathcal{Q}}_{2}}^{U}\left(y_{2} z_{2}\right) \\
& +\sum_{y_{2}=z_{2}, y_{1} z_{1} \in E_{1}} \tilde{\mu}_{\tilde{\mathcal{P}}_{2}}^{U}\left(z_{2}\right) \wedge \tilde{\mu}_{\tilde{\mathcal{Q}}_{1}}^{U}\left(y_{1} z_{1}\right) \\
& +\sum_{y_{1} z_{1} \in E_{1}, y_{2} z_{2} \in E_{2}} \tilde{\mu}_{\tilde{\mathcal{Q}}_{1}}^{U}\left(y_{1} z_{1}\right) \wedge \tilde{\mu}_{\tilde{\mathcal{Q}}_{2}}^{U}\left(y_{2} z_{2}\right), \\
& \left(\mathrm{d}_{\tilde{v} L}\right)_{\tilde{\mathcal{G}}_{1}} \otimes \tilde{\mathcal{G}}_{2}\left(z_{1}, z_{2}\right) \\
& =\sum_{\left(y_{1}, y_{2}\right)\left(z_{1}, z_{2}\right) \in E_{1} \otimes E_{2}}\left(\tilde{v}_{\tilde{\mathcal{Q}}_{1}}^{L} \otimes \tilde{v}_{\tilde{\mathcal{Q}}_{2}}^{L}\right)\left(\left(y_{1}, y_{2}\right)\left(z_{1}, z_{2}\right)\right) \\
& =\sum_{y_{1}=z_{1}, y_{2} z_{2} \in E_{2}} \tilde{v}_{\tilde{\mathcal{P}}_{1}}^{L}\left(z_{1}\right) \vee \tilde{v}_{\tilde{\mathcal{Q}}_{2}}^{L}\left(y_{2} z_{2}\right) \\
& +\sum_{y_{2}=z_{2}, y_{1} z_{1} \in E_{1}} \tilde{v}_{\tilde{\mathcal{P}}_{2}}^{L}\left(z_{2}\right) \vee \tilde{v}_{\tilde{\mathcal{Q}}_{1}}^{L}\left(y_{1} z_{1}\right) \\
& +\sum_{y_{1} z_{1} \in E_{1}, y_{2} z_{2} \in E_{2}} \tilde{v}_{\tilde{\mathcal{Q}}_{1}}^{L}\left(y_{1} z_{1}\right) \vee \tilde{v}_{\tilde{\mathcal{Q}}_{2}}^{L}\left(y_{2} z_{2}\right) .
\end{aligned}
$$

Theorem 8 Let $\tilde{\mathcal{G}}_{1}$ and $\tilde{\mathcal{G}}_{2}$ be two SIVPFGs. If $\tilde{\mu}_{\tilde{\mathcal{P}}_{1}}^{L} \geq$ $\tilde{\mu}_{\tilde{\mathcal{Q}}_{2}}^{L}, \tilde{v}_{\tilde{\mathcal{P}}_{1}}^{U} \leq \tilde{v}_{\tilde{\mathcal{Q}}_{2}}^{U}, \tilde{\mu}_{\tilde{\mathcal{P}}_{1}}^{U} \geq \tilde{\mu}_{\tilde{\mathcal{Q}}_{2}}^{U}, \tilde{v}_{\tilde{\mathcal{P}}_{1}}^{L} \leq \tilde{v}_{\tilde{\mathcal{Q}}_{2}}^{L}, \tilde{\mu}_{\tilde{\mathcal{P}}_{2}}^{L} \geq$ $\tilde{\mu}_{\tilde{\mathcal{Q}}_{1}}^{L}, \tilde{v}_{\tilde{\mathcal{P}}_{2}}^{U} \leq \tilde{v}_{\tilde{\mathcal{Q}}_{1}}^{U}, \tilde{\mu}_{\tilde{\mathcal{P}}_{2}}^{U} \geq \tilde{\mu}_{\tilde{\mathcal{Q}}_{1}}^{U}, \tilde{v}_{\tilde{\mathcal{P}}_{2}}^{L} \leq \tilde{v}_{\tilde{\mathcal{Q}}_{1}}^{L^{L}}, \tilde{\mu}_{\tilde{\mathcal{Q}}_{1}}^{L} \leq$ $\tilde{\mu}_{\tilde{\mathcal{Q}}_{2}}^{L}, \tilde{v}_{\tilde{\mathcal{Q}}_{1}}^{U} \geq \tilde{v}_{\tilde{\mathcal{Q}}_{2}}^{U}, \tilde{\mu}_{\tilde{\mathcal{Q}}_{1}}^{U} \leq \tilde{\mu}_{\tilde{\mathcal{Q}}_{2}}^{U}, \tilde{v}_{\tilde{\mathcal{Q}}_{1}}^{L} \geq \tilde{v}_{\tilde{\mathcal{Q}}_{2}}^{L}$. Then for all $\left(z_{1}, z_{2}\right) \in V_{1} \times V_{2}, \mathrm{~d}_{\tilde{\mathcal{G}}_{1}} \otimes \tilde{\mathcal{G}}_{2}\left(z_{1}, z_{2}\right)=r_{2} \mathrm{~d}_{\tilde{\mathcal{G}}_{1}}\left(z_{1}\right)+\mathrm{d}_{\tilde{\mathcal{G}}_{2}}\left(z_{2}\right)$.

Proof Using the definition of vertex degree of $\tilde{\mathcal{G}}_{1} \otimes \tilde{\mathcal{G}}_{2}$, we have

$$
\begin{aligned}
& \left(d_{\tilde{\mu}^{L}}\right)_{\tilde{\mathcal{G}}_{1} \otimes \tilde{\mathcal{G}}_{2}}\left(z_{1}, z_{2}\right) \\
& =\sum_{\left(y_{1}, y_{2}\right)\left(z_{1}, z_{2}\right) \in E_{1} \otimes E_{2}}\left(\tilde{\mu}_{\tilde{\mathcal{Q}}_{1}}^{L} \otimes \tilde{\mu}_{\tilde{\mathcal{Q}}_{2}}^{L}\right)\left(\left(y_{1}, y_{2}\right)\left(z_{1}, z_{2}\right)\right) \\
& =\sum_{z_{1}=y_{1}, z_{2} y_{2} \in E_{2}} \tilde{\mu}_{\tilde{\mathcal{P}}_{1}}^{L}\left(y_{1}\right) \wedge \tilde{\mu}_{\tilde{\mathcal{Q}}_{2}}^{L}\left(y_{2} z_{2}\right) \\
& +\sum_{z_{2}=y_{2}, y_{1} z_{1} \in E_{1}} \tilde{\mu}_{\tilde{\mathcal{P}}_{2}}^{L}\left(y_{2}\right) \wedge \tilde{\mu}_{\tilde{\mathcal{Q}}_{1}}^{L}\left(y_{1} z_{1}\right) \\
& +\sum_{y_{1} z_{1} \in E_{1}, y_{2} z_{2} \in E_{2}} \tilde{\mu}_{\tilde{\mathcal{Q}}_{1}}^{L}\left(y_{1} z_{1}\right) \wedge \tilde{\mu}_{\tilde{\mathcal{Q}}_{2}}^{L}\left(y_{2} z_{2}\right) \\
& =\sum_{z_{2} y_{2} \in E_{2}} \tilde{\mu}_{\tilde{\mathcal{Q}}_{2}}^{L}\left(y_{2} z_{2}\right)+\sum_{y_{1} z_{1} \in E_{1}} \tilde{\mu}_{\tilde{\mathcal{Q}}_{1}}^{L}\left(y_{1} z_{1}\right)+\sum_{y_{1} z_{1} \in E_{1}} \tilde{\mu}_{\tilde{\mathcal{Q}}_{1}}^{L}\left(y_{1} z_{1}\right) \\
& \text { (since } \tilde{\mu}_{\tilde{\mathcal{P}}_{1}}^{L} \geq \tilde{\mu}_{\tilde{\mathcal{Q}}_{2}}^{L}, \tilde{\mu}_{\tilde{\mathcal{P}}_{2}}^{L} \geq \tilde{\mu}_{\tilde{\mathcal{Q}}_{1}}^{L} \text { and } \tilde{\mu}_{\tilde{\mathcal{Q}}_{1}}^{L} \leq \tilde{\mu}_{\tilde{\mathcal{Q}}_{2}}^{L} \text { ) } \\
& =r_{2}\left(d_{\tilde{\mu}^{L}}\right)_{\tilde{\mathcal{G}}_{1}}\left(z_{1}\right)+\left(d_{\tilde{\mu}^{L}}\right)_{\tilde{\mathcal{G}}_{2}}\left(z_{2}\right) \text {. }
\end{aligned}
$$

Analogously, it is easy to show that $\left(d_{\tilde{\nu}^{U}}\right)_{\tilde{\mathcal{G}}_{1}} \otimes \tilde{\mathcal{G}}_{2}\left(z_{1}, z_{2}\right)=$ $r_{2}\left(d_{\tilde{v}^{U}}\right)_{\tilde{\mathcal{G}}_{1}}\left(z_{1}\right)+\left(d_{\tilde{v}^{U}}\right)_{\tilde{\mathcal{G}}_{2}}\left(z_{2}\right), \quad\left(d_{\tilde{\mu}^{U}}\right)_{\tilde{\mathcal{G}}_{1} \otimes \tilde{\mathcal{G}}_{2}}\left(z_{1}, z_{2}\right)=$ $r_{2}\left(d_{\tilde{\mu}^{U}}\right)_{\tilde{\mathcal{G}}_{1}}\left(z_{1}\right)+\left(d_{\tilde{\mu}^{U}}\right)_{\tilde{\mathcal{G}}_{2}}\left(z_{2}\right)$ and $\left(d_{\tilde{v}^{L}}\right)_{\tilde{\mathcal{G}}_{1} \otimes \tilde{\mathcal{G}}_{2}}\left(z_{1}, z_{2}\right)=$ 
$r_{2}\left(d_{\tilde{v}^{L}}\right)_{\tilde{\mathcal{G}}_{1}}\left(z_{1}\right)+\left(d_{\tilde{v}^{L}}\right)_{\tilde{\mathcal{G}}_{2}}\left(z_{2}\right)$. Hence $d_{\tilde{\mathcal{G}}_{1} \otimes \tilde{\mathcal{G}}_{2}}\left(z_{1}, z_{2}\right)=$ $r_{2} d_{\tilde{\mathcal{G}}_{1}}\left(z_{1}\right)+d_{\tilde{\mathcal{G}}_{2}}\left(z_{2}\right)$.

Theorem 9 Let $\tilde{\mathcal{G}}_{1}$ and $\tilde{\mathcal{G}}_{2}$ be two SIVPFGs. If

(i) $\tilde{\mu}_{\tilde{\mathcal{P}}_{1}}^{L} \geq \tilde{\mu}_{\tilde{\mathcal{Q}}_{2}}^{L}, \tilde{\mu}_{\tilde{\mathcal{P}}_{2}}^{L} \geq \tilde{\mu}_{\tilde{\mathcal{Q}}_{1}}^{L}, \tilde{\mu}_{\tilde{\mathcal{Q}}_{1}}^{L} \leq \tilde{\mu}_{\tilde{\mathcal{Q}}_{2}}^{L}$, then $\left(t \mathrm{~d}_{\tilde{\mu}^{L}}\right)_{\tilde{\mathcal{G}}_{1} \otimes \tilde{\mathcal{G}}_{2}}\left(z_{1}, z_{2}\right)=\left(t \mathrm{~d}_{\tilde{\mu}^{L}}\right)_{\tilde{\mathcal{G}}_{2}}\left(z_{2}\right)+r_{2}\left(t \mathrm{~d}_{\tilde{\mu}^{L}}\right)_{\tilde{\mathcal{G}}_{1}}\left(z_{1}\right)$ $-\left(r_{2}-1\right) \tilde{\mu}_{\tilde{\mathcal{P}}_{1}}^{L}\left(z_{1}\right)-\tilde{\mu}_{\tilde{\mathcal{P}}_{1}}^{L}\left(z_{1}\right) \vee \tilde{\mu}_{\tilde{\mathcal{P}}_{2}}^{L}\left(z_{2}\right)$;

(ii) $\tilde{v}_{\tilde{\mathcal{P}}_{1}}^{U} \leq \tilde{v}_{\tilde{\mathcal{Q}}_{2}}^{U}, \tilde{v}_{\tilde{\mathcal{P}}_{2}}^{U} \leq \tilde{v}_{\tilde{\mathcal{Q}}_{1}}^{U}, \tilde{\mu}_{\tilde{\mathcal{Q}}_{1}}^{U} \geq \tilde{\mu}_{\tilde{\mathcal{Q}}_{2}}^{U}$, then $\left(t \mathrm{~d}_{\tilde{v} U}\right)_{\tilde{\mathcal{G}}_{1} \otimes \tilde{\mathcal{G}}_{2}}\left(z_{1}, z_{2}\right)=\left(t \mathrm{~d}_{\tilde{v} U}\right)_{\tilde{\mathcal{G}}_{2}}\left(z_{2}\right)+r_{2}\left(t \mathrm{~d}_{\tilde{v}^{U} U}\right)_{\tilde{\mathcal{G}}_{1}}\left(z_{1}\right)$ $-\left(r_{2}-1\right) \tilde{v}_{\tilde{\mathcal{P}}_{1}}^{U}\left(z_{1}\right)-\tilde{v}_{\tilde{\mathcal{P}}_{1}}^{U}\left(z_{1}\right) \wedge \tilde{v}_{\tilde{\mathcal{P}}_{2}}^{U}\left(z_{2}\right) ;$

(iii) $\tilde{\mu}_{\tilde{\mathcal{P}}_{1}}^{U} \geq \tilde{\mu}_{\tilde{\mathcal{Q}}_{2}}^{U}, \tilde{v}_{\tilde{\mathcal{P}}_{2}}^{U} \geq \tilde{v}_{\tilde{\mathcal{Q}}_{1}}^{U}, \tilde{\mu}_{\tilde{\mathcal{Q}}_{1}}^{U} \leq \tilde{\mu}_{\tilde{\mathcal{Q}}_{2}}^{U}$, then $\left(t \mathrm{~d}_{\tilde{\mu}^{U}}\right)_{\tilde{\mathcal{G}}_{1}} \otimes \tilde{\mathcal{G}}_{2}\left(z_{1}, z_{2}\right)=\left(t \mathrm{~d}_{\tilde{\mu}^{U}}\right)_{\tilde{\mathcal{G}}_{2}}\left(z_{2}\right)+r_{2}\left(t \mathrm{~d}_{\tilde{\mu}^{U}}\right)_{\tilde{\mathcal{G}}_{1}}\left(z_{1}\right)$ $-\left(r_{2}-1\right) \tilde{\mu}_{\tilde{\mathcal{P}}_{1}}^{U}\left(z_{1}\right)-\tilde{\mu}_{\tilde{\mathcal{P}}_{1}}^{U}\left(z_{1}\right) \vee \tilde{\mu}_{\tilde{\mathcal{P}}_{2}}^{U}\left(z_{2}\right)$;

(iv) $\tilde{v}_{\tilde{\mathcal{P}}_{1}}^{L} \leq \tilde{v}_{\tilde{\mathcal{Q}}_{2}}^{L}, \quad \tilde{v}_{\tilde{\mathcal{P}}_{2}}^{L} \leq \tilde{v}_{\tilde{\mathcal{Q}}_{1}}^{L}, \tilde{v}_{\tilde{\mathcal{Q}}_{1}}^{L} \geq \tilde{v}_{\tilde{\mathcal{Q}}_{2}}^{L}$, then $\left(t \mathrm{~d}_{\tilde{v}^{L}}\right)_{\tilde{\mathcal{G}}_{1} \otimes \tilde{\mathcal{G}}_{2}}\left(z_{1}, z_{2}\right)=\left(t \mathrm{~d}_{\tilde{v}^{L}}\right)_{\tilde{\mathcal{G}}_{2}}\left(z_{2}\right)+r_{2}\left(t \mathrm{~d}_{\tilde{v}^{L}}\right)_{\tilde{\mathcal{G}}_{1}}\left(z_{1}\right)-$ $\left(r_{2}-1\right) \tilde{v}_{\tilde{\mathcal{P}}_{1}}^{L}\left(z_{1}\right)-\tilde{v}_{\tilde{\mathcal{P}}_{1}}^{L}\left(z_{1}\right) \wedge \tilde{v}_{\tilde{\mathcal{P}}_{2}}^{L}\left(z_{2}\right)$

for all $\left(z_{1}, z_{2}\right) \in V_{1} \times V_{2}$.

Proof For any vertex $\left(z_{1}, z_{2}\right) \in V_{1} \times V_{2}$,

(i) If $\tilde{\mu}_{\tilde{\mathcal{P}}_{1}}^{L} \geq \tilde{\mu}_{\tilde{\mathcal{Q}}_{2}}^{L}, \tilde{\mu}_{\tilde{\mathcal{P}}_{2}}^{L} \geq \tilde{\mu}_{\tilde{\mathcal{Q}}_{1}}^{L}, \tilde{\mu}_{\tilde{\mathcal{Q}}_{1}}^{L} \leq \tilde{\mu}_{\tilde{\mathcal{Q}}_{2}}^{L}$

$$
\begin{aligned}
( & t \mathrm{~d}_{\tilde{\mu}^{L}}^{L} \tilde{\mathcal{\mathcal { G }}}_{1} \otimes \tilde{\mathcal{G}}_{2}\left(z_{1}, z_{2}\right) \\
= & \sum_{y_{1}=z_{1}, y_{2} z_{2} \in E_{2}} \tilde{\mu}_{\tilde{\mathcal{P}}_{1}}^{L}\left(z_{1}\right) \wedge \tilde{\mu}_{\tilde{\mathcal{Q}}_{2}}^{L}\left(y_{2} z_{2}\right) \\
& +\sum_{y_{2}=z_{2}, y_{1} z_{1} \in E_{1}} \tilde{\mu}_{\tilde{\mathcal{P}}_{2}}^{L}\left(z_{2}\right) \wedge \tilde{\mu}_{\tilde{\mathcal{Q}}_{1}}^{L}\left(y_{1} z_{1}\right) \\
& +\sum_{y_{1} z_{1} \in E_{1}, y_{2} z_{2} \in E_{2}} \tilde{\mu}_{\tilde{\mathcal{Q}}_{1}}^{L}\left(y_{1} z_{1}\right) \wedge \tilde{\mu}_{\tilde{\mathcal{Q}}_{2}}^{L}\left(y_{2} z_{2}\right) \\
& +\tilde{\mu}_{\tilde{\mathcal{P}}_{1}}^{L}\left(z_{1}\right) \wedge \tilde{\mu}_{\tilde{\mathcal{P}}_{2}}^{L}\left(z_{2}\right) \\
= & \sum_{y_{2} z_{2} \in E_{2}} \tilde{\mu}_{\tilde{\mathcal{Q}}_{2}}^{L}\left(y_{2} z_{2}\right) \\
& +\sum_{y_{1} z_{1} \in E_{1}} \tilde{\mu}_{\tilde{\mathcal{Q}}_{1}}^{L}\left(y_{1} z_{1}\right)+\sum_{y_{1} z_{1} \in E_{1}} \tilde{\mu}_{\tilde{\mathcal{Q}}_{1}}^{L}\left(y_{1} z_{1}\right) \\
& +\tilde{\mu}_{\tilde{\mathcal{P}}_{1}}^{L}\left(z_{1}\right)+\tilde{\mu}_{\tilde{\mathcal{P}}_{2}}^{L}\left(z_{2}\right)-\tilde{\mu}_{\tilde{\mathcal{P}}_{1}}^{L}\left(z_{1}\right) \vee \tilde{\mu}_{\tilde{\mathcal{P}}_{2}}^{L}\left(z_{2}\right) \\
= & \sum_{y_{2} z_{2} \in E_{2}} \tilde{\mu}_{\tilde{\mathcal{Q}}_{2}}^{L}\left(y_{2} z_{2}\right) \\
& +\tilde{\mu}_{\tilde{\mathcal{P}}_{2}}^{L}\left(z_{2}\right)+r_{2} \sum_{y_{1} z_{1} \in E_{1}} \tilde{\mu}_{\tilde{\mathcal{Q}}_{1}}^{L}\left(y_{1} z_{1}\right)+\tilde{\mu}_{\tilde{\mathcal{P}}_{1}}^{L}\left(z_{1}\right) \\
& -\tilde{\mu}_{\tilde{\mathcal{P}}_{1}}^{L}\left(z_{1}\right) \vee \tilde{\mu}_{\tilde{\mathcal{P}}_{2}}^{L}\left(z_{2}\right) \\
= & \sum_{y_{2} z_{2} \in E_{2}} \tilde{\mu}_{\tilde{\mathcal{Q}}_{2}}^{L}\left(y_{2} z_{2}\right)
\end{aligned}
$$

$$
\begin{aligned}
& +\tilde{\mu}_{\tilde{\mathcal{P}}_{2}}^{L}\left(z_{2}\right)+r_{2}\left(\sum_{y_{1} z_{1} \in E_{1}} \tilde{\mu}_{\tilde{\mathcal{Q}}_{1}}^{L}\left(y_{1} z_{1}\right)+\tilde{\mu}_{\tilde{\mathcal{P}}_{1}}^{L}\left(z_{1}\right)\right) \\
& -\left(r_{2}-1\right) \tilde{\mu}_{\tilde{\mathcal{P}}_{1}}^{L}\left(z_{1}\right)-\tilde{\mu}_{\tilde{\mathcal{P}}_{1}}^{L}\left(z_{1}\right) \vee \tilde{\mu}_{\tilde{\mathcal{P}}_{2}}^{L}\left(z_{2}\right) \\
= & \left(t \mathrm{~d}_{\tilde{\mu}^{L}}\right)_{\tilde{\mathcal{G}}_{2}}\left(z_{2}\right)+r_{2}\left(t \mathrm{~d}_{\tilde{\mu}^{L}}\right)_{\tilde{\mathcal{G}}_{1}}\left(z_{1}\right)-\left(r_{2}-1\right) \tilde{\mu}_{\tilde{\mathcal{P}}_{1}}^{L}\left(z_{1}\right) \\
& -\tilde{\mu}_{\tilde{\mathcal{P}}_{1}}^{L}\left(z_{1}\right) \vee \tilde{\mu}_{\tilde{\mathcal{P}}_{2}}^{L}\left(z_{2}\right) .
\end{aligned}
$$

Analogously, we can prove (ii)-(iv).

Definition 21 Let $\tilde{\mathcal{G}}_{1}$ and $\tilde{\mathcal{G}}_{2}$ be two SIVPFGs of $G_{1}$ and $G_{2}$, respectively. The lexicographic product of these two SIVPFGs is denoted by $\tilde{\mathcal{G}}_{1}\left[\tilde{\mathcal{G}}_{2}\right]=\left(\tilde{\mathcal{P}}_{1} \circ \tilde{\mathcal{P}}_{2}, \tilde{\mathcal{Q}}_{1} \circ \tilde{\mathcal{Q}}_{2}\right)$ and defined as follows:

(i)

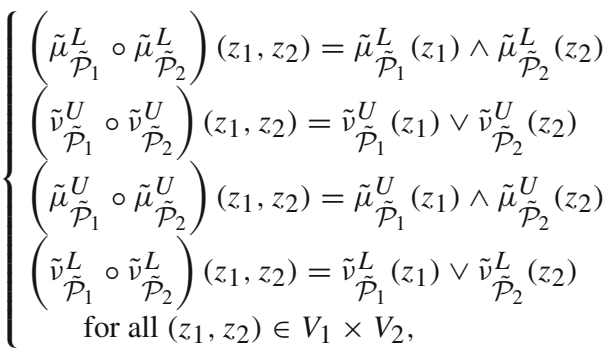

(ii)

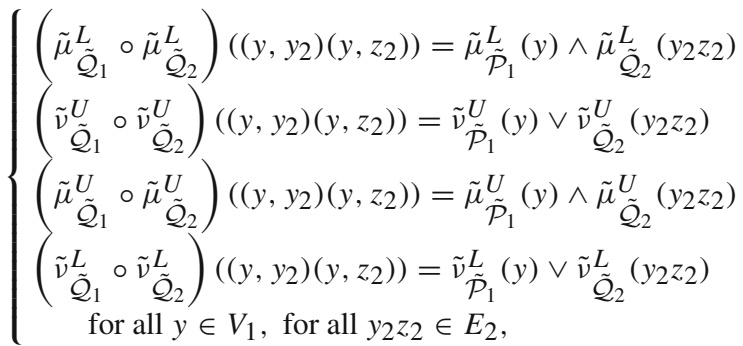

$\left(\tilde{\mu}_{\tilde{\mathcal{Q}}_{1}}^{L} \circ \tilde{\mu}_{\tilde{\mathcal{Q}}_{2}}^{L}\right)\left(\left(y_{1}, z\right)\left(z_{1}, z\right)\right)=\tilde{\mu}_{\tilde{\mathcal{Q}}_{1}}^{L}\left(y_{1} z_{1}\right) \wedge \tilde{\mu}_{\tilde{\mathcal{P}}_{2}}^{L}(z)$

$\left(\tilde{v}_{\tilde{\mathcal{Q}}_{1}}^{U} \circ \tilde{v}_{\tilde{\mathcal{Q}}_{2}}^{U}\right)\left(\left(y_{1}, z\right)\left(z_{1}, z\right)\right)=\tilde{v}_{\tilde{\mathcal{Q}}_{1}}^{U}\left(y_{1} z_{1}\right) \vee \tilde{v}_{\tilde{\mathcal{P}}_{2}}^{U}(z)$

(iii)

$\left(\tilde{\mu}_{\tilde{\mathcal{Q}}_{1}}^{U} \circ \tilde{\mu}_{\tilde{\mathcal{Q}}_{2}}^{U}\right)\left(\left(y_{1}, z\right)\left(z_{1}, z\right)\right)=\tilde{\mu}_{\tilde{\mathcal{Q}}_{1}}^{U}\left(y_{1} z_{1}\right) \wedge \tilde{\mu}_{\tilde{\mathcal{P}}_{2}}^{U}(z)$

$\left(\tilde{v}_{\tilde{\mathcal{Q}}_{1}}^{L} \circ \tilde{v}_{\tilde{\mathcal{Q}}_{2}}^{L}\right)\left(\left(y_{1}, z\right)\left(z_{1}, z\right)\right)=\tilde{v}_{\tilde{\mathcal{Q}}_{1}}^{L}\left(y_{1} z_{1}\right) \vee \tilde{v}_{\tilde{\mathcal{P}}_{2}}^{L}(z)$ for all $z \in V_{2}$, for all $y_{1} z_{1} \in E_{1}$,

(iv) $\left\{\begin{array}{c}\left(\tilde{\mu}_{\tilde{\mathcal{Q}}_{1}}^{L} \circ \tilde{\mu}_{\tilde{\mathcal{Q}}_{2}}^{L}\right)\left(\left(y_{1}, y_{2}\right)\left(z_{1}, z_{2}\right)\right) \\ =\tilde{\mu}_{\tilde{\mathcal{P}}_{2}}^{L}\left(y_{2}\right) \wedge \tilde{\mu}_{\tilde{\mathcal{P}}_{2}}^{L}\left(z_{2}\right) \wedge \tilde{\mu}_{\tilde{\mathcal{Q}}_{1}}^{L}\left(y_{1} z_{1}\right) \\ \left(\tilde{v}_{\tilde{\mathcal{Q}}_{1}}^{U} \circ \tilde{v}_{\tilde{\mathcal{Q}}_{2}}^{U}\right)\left(\left(y_{1}, y_{2}\right)\left(z_{1}, z_{2}\right)\right) \\ =\tilde{v}_{\tilde{\mathcal{P}}_{2}}^{U}\left(y_{2}\right) \vee \tilde{v}_{\tilde{\mathcal{P}}_{2}}^{U}\left(z_{2}\right) \vee \tilde{v}_{\tilde{\mathcal{Q}}_{1}}^{U}\left(y_{1} z_{1}\right) \\ \left(\tilde{\mu}_{\tilde{\mathcal{Q}}_{1}}^{U} \circ \tilde{\mu}_{\tilde{\mathcal{Q}}_{2}}^{U}\right)\left(\left(y_{1}, y_{2}\right)\left(z_{1}, z_{2}\right)\right) \\ =\tilde{\mu}_{\tilde{\mathcal{P}}_{2}}^{U}\left(y_{2}\right) \wedge \tilde{\mu}_{\tilde{\mathcal{P}}_{2}}^{U}\left(z_{2}\right) \wedge \tilde{\mu}_{\tilde{\mathcal{Q}}_{1}}^{U}\left(y_{1} z_{1}\right) \\ \left(\tilde{v}_{\tilde{\mathcal{Q}}_{1}}^{L} \circ \tilde{v}_{\tilde{\mathcal{Q}}_{2}}^{L}\right)\left(\left(y_{1}, y_{2}\right)\left(z_{1}, z_{2}\right)\right) \\ =\tilde{v}_{\tilde{\mathcal{P}}_{2}}^{L}\left(y_{2}\right) \vee \tilde{v}_{\tilde{\mathcal{P}}_{2}}^{L}\left(z_{2}\right) \vee \tilde{v}_{\tilde{\mathcal{Q}}_{1}}^{L}\left(y_{1} z_{1}\right) \\ \quad \text { or all } y_{1} z_{1} \in E_{1}, z_{2} \neq y_{2} .\end{array}\right.$ 
Proposition 5 The lexicographic product $\tilde{\mathcal{G}}_{1}\left[\tilde{\mathcal{G}}_{2}\right]$ of two SIVPFGs of $G_{1}$ and $G_{2}$ is a SIVPFG of $G_{1}\left[G_{2}\right]$.

Definition 22 Let $\tilde{\mathcal{G}}_{1}$ and $\tilde{\mathcal{G}}_{2}$ be two SIVPFGs. For any vertex $\left(z_{1}, z_{2}\right) \in V_{1} \times V_{2}$,

$$
\begin{aligned}
& \left(\mathrm{d}_{\left.\tilde{\mu}^{L}\right)} \tilde{\mathcal{G}}_{1}\left[\tilde{\mathcal{G}}_{2}\right]\right. \\
& =\sum_{\left(y_{1}, y_{2}\right)\left(z_{1}, z_{2}\right) \in E_{1} \circ E_{2}}\left(\tilde{\mu}_{\tilde{\mathcal{Q}}_{1}}^{L} \circ \tilde{\mu}_{\tilde{\mathcal{Q}}_{2}}^{L}\right)\left(\left(y_{1}, y_{2}\right)\left(z_{1}, z_{2}\right)\right) \\
& =\sum_{y_{1}=z_{1}, y_{2} z_{2} \in E_{2}} \tilde{\mu}_{\tilde{\mathcal{P}}_{1}}^{L}\left(z_{1}\right) \wedge \tilde{\mu}_{\tilde{\mathcal{Q}}_{2}}^{L}\left(y_{2} z_{2}\right) \\
& +\sum_{y_{2}=z_{2}, y_{1} z_{1} \in E_{1}} \tilde{\mu}_{\tilde{\mathcal{P}}_{2}}^{L}\left(z_{2}\right) \wedge \tilde{\mu}_{\tilde{\mathcal{Q}}_{1}}^{L}\left(y_{1} z_{1}\right) \\
& +\sum_{z_{2} \neq y_{2}, y_{1} z_{1} \in E_{1}} \tilde{\mu}_{\tilde{\mathcal{P}}_{2}}^{L}\left(y_{2}\right) \wedge \tilde{\mu}_{\tilde{\mathcal{P}}_{2}}^{L}\left(z_{2}\right) \wedge \tilde{\mu}_{\tilde{\mathcal{Q}}_{1}}^{L}\left(y_{1} z_{1}\right), \\
& \left(\mathrm{d}_{\tilde{v}^{U}}\right)_{\tilde{\mathcal{G}}_{1}\left[\tilde{\mathcal{G}}_{2}\right]}\left(z_{1}, z_{2}\right) \\
& =\sum_{\left(y_{1}, y_{2}\right)\left(z_{1}, z_{2}\right) \in E_{1} \circ E_{2}}\left(\tilde{v}_{\tilde{\mathcal{Q}}_{1}}^{U} \circ \tilde{v}_{\tilde{\mathcal{Q}}_{2}}^{U}\right)\left(\left(y_{1}, y_{2}\right)\left(z_{1}, z_{2}\right)\right) \\
& =\sum_{y_{1}=z_{1}, y_{2} z_{2} \in E_{2}} \tilde{v}_{\tilde{\mathcal{P}}_{1}}^{U}\left(z_{1}\right) \vee \tilde{v}_{\tilde{\mathcal{Q}}_{2}}^{U}\left(y_{2} z_{2}\right) \\
& +\sum_{y_{2}=z_{2}, y_{1} z_{1} \in E_{1}} \tilde{v}_{\tilde{\mathcal{P}}_{2}}^{U}\left(z_{2}\right) \vee \tilde{v}_{\tilde{\mathcal{Q}}_{1}}^{U}\left(y_{1} z_{1}\right) \\
& +\sum_{z_{2} \neq y_{2}, y_{1} z_{1} \in E_{1}} \tilde{v}_{\tilde{\mathcal{P}}_{2}}^{U}\left(y_{2}\right) \vee \tilde{v}_{\tilde{\mathcal{P}}_{2}}^{U}\left(z_{2}\right) \vee \tilde{v}_{\tilde{\mathcal{Q}}_{1}}^{U}\left(y_{1} z_{1}\right), \\
& \left(\mathrm{d}_{\tilde{\mu}^{U}}\right)_{\tilde{\mathcal{G}}_{1}\left[\tilde{\mathcal{G}}_{2}\right]}\left(z_{1}, z_{2}\right) \\
& =\sum_{\left(y_{1}, y_{2}\right)\left(z_{1}, z_{2}\right) \in E_{1} \circ E_{2}}\left(\tilde{\mu}_{\tilde{\mathcal{Q}}_{1}}^{U} \circ \tilde{\mu}_{\tilde{\mathcal{Q}}_{2}}^{U}\right)\left(\left(y_{1}, y_{2}\right)\left(z_{1}, z_{2}\right)\right) \\
& =\sum_{y_{1}=z_{1}, y_{2} z_{2} \in E_{2}} \tilde{\mu}_{\tilde{\mathcal{P}}_{1}}^{U}\left(z_{1}\right) \wedge \tilde{\mu}_{\tilde{\mathcal{Q}}_{2}}^{U}\left(y_{2} z_{2}\right) \\
& +\sum_{y_{2}=z_{2}, y_{1} z_{1} \in E_{1}} \tilde{\mu}_{\tilde{\mathcal{P}}_{2}}^{U}\left(z_{2}\right) \wedge \tilde{\mu}_{\tilde{\mathcal{Q}}_{1}}^{U}\left(y_{1} z_{1}\right) \\
& +\sum_{z_{2} \neq y_{2}, y_{1} z_{1} \in E_{1}} \tilde{\mu}_{\tilde{\mathcal{P}}_{2}}^{U}\left(y_{2}\right) \wedge \tilde{\mu}_{\tilde{\mathcal{P}}_{2}}^{U}\left(z_{2}\right) \wedge \tilde{\mu}_{\tilde{\mathcal{Q}}_{1}}^{U}\left(y_{1} z_{1}\right), \\
& \left(\mathrm{d}_{\tilde{\nu} L}\right)_{\tilde{\mathcal{G}}_{1}\left[\tilde{\mathcal{G}}_{2}\right]}\left(z_{1}, z_{2}\right) \\
& =\sum_{\left(y_{1}, y_{2}\right)\left(z_{1}, z_{2}\right) \in E_{1} \circ E_{2}}\left(\tilde{v}_{\tilde{\mathcal{Q}}_{1}}^{L} \circ \tilde{v}_{\tilde{\mathcal{Q}}_{2}}^{L}\right)\left(\left(y_{1}, y_{2}\right)\left(z_{1}, z_{2}\right)\right) \\
& =\sum_{y_{1}=z_{1}, y_{2} z_{2} \in E_{2}} \tilde{v}_{\tilde{\mathcal{P}}_{1}}^{L}\left(z_{1}\right) \vee \tilde{v}_{\tilde{\mathcal{Q}}_{2}}^{L}\left(y_{2} z_{2}\right) \\
& +\sum_{y_{2}=z_{2}, y_{1} z_{1} \in E_{1}} \tilde{v}_{\tilde{\mathcal{P}}_{2}}^{L}\left(z_{2}\right) \vee \tilde{v}_{\tilde{\mathcal{Q}}_{1}}^{L}\left(y_{1} z_{1}\right) \\
& +\sum_{z_{2} \neq y_{2}, y_{1} z_{1} \in E_{1}} \tilde{v}_{\tilde{\mathcal{P}}_{2}}^{L}\left(y_{2}\right) \vee \tilde{v}_{\tilde{\mathcal{P}}_{2}}^{L}\left(z_{2}\right) \vee \tilde{v}_{\tilde{\mathcal{Q}}_{1}}^{L}\left(y_{1} z_{1}\right) .
\end{aligned}
$$

Theorem 10 Let $\tilde{\mathcal{G}}_{1}$ and $\tilde{\mathcal{G}}_{2}$ be two SIVPFGs. If $\tilde{\mu}_{\tilde{\mathcal{P}}_{1}}^{L} \geq$ $\tilde{\mu}_{\tilde{\mathcal{Q}}_{2}}^{L}, \tilde{v}_{\tilde{\mathcal{P}}_{1}}^{U} \leq \tilde{v}_{\tilde{\mathcal{Q}}_{2}}^{U}, \tilde{\mu}_{\tilde{\mathcal{P}}_{1}}^{U} \geq \tilde{\mu}_{\tilde{\mathcal{Q}}_{2}}^{U}, \tilde{v}_{\tilde{\mathcal{P}}_{1}}^{L} \leq \tilde{v}_{\tilde{\mathcal{Q}}_{2}}^{L}$ and $\tilde{\mu}_{\tilde{\mathcal{P}}_{2}}^{L} \geq$ $\tilde{\mu}_{\tilde{\mathcal{Q}}_{1}}^{L}, \tilde{v}_{\tilde{\mathcal{P}}_{2}}^{U} \leq \tilde{v}_{\tilde{\mathcal{Q}}_{1}}^{U}, \tilde{\mu}_{\tilde{\mathcal{P}}_{2}}^{U} \geq \tilde{\mu}_{\tilde{\mathcal{Q}}_{1}}^{U}, \tilde{v}_{\tilde{\mathcal{P}}_{2}}^{L} \leq \tilde{\boldsymbol{v}}_{\tilde{\mathcal{Q}}_{1}}^{L}$. Then $\mathrm{d}_{\tilde{\mathcal{G}}_{1}\left[\tilde{\mathcal{G}}_{2}\right]}\left(z_{1}, z_{2}\right)=r_{2} \mathrm{~d}_{\tilde{\mathcal{G}}_{1}}\left(z_{1}\right)+\mathrm{d}_{\tilde{\mathcal{G}}_{2}}\left(z_{2}\right)$ for all $\left(z_{1}, z_{2}\right) \in$ $V_{1} \times V_{2}$.
Proof For any vertex $\left(z_{1}, z_{2}\right) \in V_{1} \times V_{2}$,

$$
\begin{aligned}
& \left(\mathrm{d}_{\tilde{\mu}^{L}}\right)_{\tilde{\mathcal{G}}_{1}\left[\tilde{\mathcal{G}}_{2}\right]}\left(z_{1}, z_{2}\right) \\
& =\sum_{\left(y_{1}, y_{2}\right)\left(z_{1}, z_{2}\right) \in E_{1} \circ E_{2}}\left(\tilde{\mu}_{\tilde{\mathcal{Q}}_{1}}^{L} \circ \tilde{\mu}_{\tilde{\mathcal{Q}}_{2}}^{L}\right)\left(\left(y_{1}, y_{2}\right)\left(z_{1}, z_{2}\right)\right) \\
& =\sum_{y_{1}=z_{1}, y_{2} z_{2} \in E_{2}} \tilde{\mu}_{\tilde{\mathcal{P}}_{1}}^{L}\left(z_{1}\right) \wedge \tilde{\mu}_{\tilde{\mathcal{Q}}_{2}}^{L}\left(y_{2} z_{2}\right) \\
& +\sum_{y_{2}=z_{2}, y_{1} z_{1} \in E_{1}} \tilde{\mu}_{\tilde{\mathcal{P}}_{2}}^{L}\left(z_{2}\right) \wedge \tilde{\mu}_{\tilde{\mathcal{Q}}_{1}}^{L}\left(y_{1} z_{1}\right) \\
& +\sum_{z_{2} \neq y_{2}, y_{1} z_{1} \in E_{1}} \tilde{\mu}_{\tilde{\mathcal{P}}_{2}}^{L}\left(y_{2}\right) \wedge \tilde{\mu}_{\tilde{\mathcal{P}}_{2}}^{L}\left(z_{2}\right) \wedge \tilde{\mu}_{\tilde{\mathcal{Q}}_{1}}^{L}\left(y_{1} z_{1}\right) \\
& =\sum_{y_{2} z_{2} \in E_{2}} \tilde{\mu}_{\tilde{\mathcal{Q}}_{2}}^{L}\left(y_{2} z_{2}\right) \\
& +\sum_{y_{1} z_{1} \in E_{1}} \tilde{\mu}_{\tilde{\mathcal{Q}}_{1}}^{L}\left(y_{1} z_{1}\right)+\sum_{y_{1} z_{1} \in E_{1}} \tilde{\mu}_{\tilde{\mathcal{Q}}_{1}}^{L}\left(y_{1} z_{1}\right) \\
& \text { (since } \tilde{\mu}_{\tilde{\mathcal{P}}_{1}}^{L} \geq \tilde{\mu}_{\tilde{\mathcal{Q}}_{2}}^{L} \text { and } \tilde{\mu}_{\tilde{\mathcal{P}}_{2}}^{L} \geq \tilde{\mu}_{\tilde{\mathcal{Q}}_{1}}^{L} \text { ) } \\
& =\left(\mathrm{d}_{\tilde{\mu}^{L}}\right)_{\tilde{\mathcal{G}}_{2}}\left(z_{2}\right)+r_{2}\left(\mathrm{~d}_{\tilde{\mu}^{L}}\right)_{\tilde{\mathcal{G}}_{1}}\left(z_{1}\right) \text {. }
\end{aligned}
$$

Analogously, we can show that $\left(\mathrm{d}_{\tilde{\nu}} U\right)_{\tilde{\mathcal{G}}_{1}\left[\tilde{\mathcal{G}}_{2}\right]}\left(z_{1}, z_{2}\right)$ $=\left(\mathrm{d}_{\tilde{\nu}} U\right)_{\tilde{\mathcal{G}}_{2}}\left(z_{2}\right)+r_{2}\left(\mathrm{~d}_{\tilde{\nu} U}\right)_{\tilde{\mathcal{G}}_{1}}\left(z_{1}\right),\left(\mathrm{d}_{\tilde{\mu}^{U}}\right)_{\tilde{\mathcal{G}}_{1}\left[\tilde{\mathcal{G}}_{2}\right]}\left(z_{1}, z_{2}\right)=$

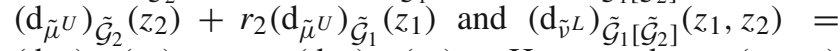
$\left(\mathrm{d}_{\tilde{v} L}\right)_{\tilde{\mathcal{G}}_{2}}\left(z_{2}\right)+r_{2}\left(\mathrm{~d}_{\tilde{v}^{L}}\right)_{\tilde{\mathcal{G}}_{1}}\left(z_{1}\right)$. Hence $\mathrm{d}_{\tilde{\mathcal{G}}_{1}\left[\tilde{\mathcal{G}}_{2}\right]}\left(z_{1}, z_{2}\right)$ $=\mathrm{d}_{\tilde{\mathcal{G}}_{2}}\left(z_{2}\right)+r_{2} \mathrm{~d}_{\tilde{\mathcal{G}}_{1}}\left(z_{1}\right)$.

Theorem 11 Let $\tilde{\mathcal{G}}_{1}$ and $\tilde{\mathcal{G}}_{2}$ be two SIVPFGs. If

(i) $\tilde{\mu}_{\tilde{\mathcal{P}}_{1}}^{L} \geq \tilde{\mu}_{\tilde{\mathcal{Q}}_{2}}^{L}$ and $\tilde{\mu}_{\tilde{\mathcal{P}}_{2}}^{L} \geq \tilde{\mu}_{\tilde{\mathcal{Q}}_{1}}^{L}$, then $\left(t \mathrm{~d}_{\tilde{\mu}^{L}}\right)_{\tilde{\mathcal{G}}_{1}\left[\tilde{\mathcal{G}}_{2]}\right.}\left(z_{1}, z_{2}\right)$ $=\left(t \mathrm{~d}_{\tilde{\mu}^{L}}\right)_{\tilde{\mathcal{G}}_{2}}\left(z_{2}\right)+r_{2}\left(t \mathrm{~d}_{\tilde{\mu}^{L}}\right)_{\tilde{\mathcal{G}}_{1}}\left(z_{1}\right)-\left(r_{2}-1\right) \tilde{\mu}_{\tilde{\mathcal{P}}_{1}}^{L}\left(z_{1}\right)-$ $\tilde{\mu}_{\tilde{\mathcal{P}}_{1}}^{L}\left(z_{1}\right) \vee \tilde{\mu}_{\tilde{\mathcal{P}}_{2}}^{L}\left(z_{2}\right)$.

(ii) $\tilde{v}_{\tilde{\mathcal{P}}_{1}}^{U} \leq \tilde{v}_{\tilde{\mathcal{Q}}_{2}}^{U}$ and $\tilde{v}_{\tilde{\mathcal{P}}_{2}}^{U} \leq \tilde{v}_{\tilde{\mathcal{Q}}_{1}}^{U}$, then $\left(t \mathrm{~d}_{\tilde{v}^{U}}\right)_{\tilde{\mathcal{G}}_{1}\left[\tilde{\mathcal{G}}_{2}\right]}\left(z_{1}, z_{2}\right)=$ $\left(t \mathrm{~d}_{\tilde{v} U}\right)_{\tilde{\mathcal{G}}_{2}}\left(z_{2}\right)+r_{2}\left(t \mathrm{~d}_{\tilde{v}^{U}}\right) \tilde{\mathcal{G}}_{1}\left(z_{1}\right)-\left(r_{2}-1\right) \tilde{v}_{\tilde{\mathcal{P}}_{1}}^{U}\left(z_{1}\right)-$ $\tilde{v}_{\tilde{\mathcal{P}}_{1}}^{U}\left(z_{1}\right) \wedge \tilde{v}_{\tilde{\mathcal{P}}_{2}}^{U}\left(z_{2}\right)$.

(iii) $\tilde{\mu}_{\tilde{\mathcal{P}}_{1}}^{U} \geq \tilde{\mu}_{\tilde{\mathcal{Q}}_{2}}^{U}$ and $\tilde{\mu}_{\tilde{\mathcal{P}}_{2}}^{U} \geq \tilde{\mu}_{\tilde{\mathcal{Q}}_{1}}^{U}$, then $\left(t \mathrm{~d}_{\tilde{\mu}^{U}}\right)_{\tilde{\mathcal{G}}_{1}\left[\tilde{\mathcal{G}}_{2}\right]}\left(z_{1}, z_{2}\right)$ $=\left(t \mathrm{~d}_{\tilde{\mu}^{U}}\right) \tilde{\mathcal{G}}_{2}\left(z_{2}\right)+r_{2}\left(t \mathrm{~d}_{\tilde{\mu}^{U}}\right) \tilde{\mathcal{G}}_{1}\left(z_{1}\right)-\left(r_{2}-1\right) \tilde{\mu}_{\tilde{\mathcal{P}}_{1}}^{U}\left(z_{1}\right)-$ $\tilde{\mu}_{\tilde{\mathcal{P}}_{1}}^{U}\left(z_{1}\right) \vee \tilde{\mu}_{\tilde{\mathcal{P}}_{2}}^{U}\left(z_{2}\right)$

(iv) $\tilde{v}_{\tilde{\mathcal{P}}_{1}}^{L} \leq \tilde{v}_{\tilde{\mathcal{Q}}_{2}}^{L}$ and $\tilde{v}_{\tilde{\mathcal{P}}_{2}}^{L} \leq \tilde{v}_{\tilde{\mathcal{Q}}_{1}}^{L}$, then $\left(t \mathrm{~d}_{\tilde{v}^{L}}\right)_{\tilde{\mathcal{G}}_{1}\left[\tilde{\mathcal{G}}_{2}\right]}\left(z_{1}, z_{2}\right)=$ $\left(t \mathrm{~d}_{\tilde{v} L}\right)_{\tilde{\mathcal{G}}_{2}}\left(z_{2}\right)+r_{2}\left(t \mathrm{~d}_{\tilde{v}^{L}}\right)_{\tilde{\mathcal{G}}_{1}}\left(z_{1}\right)-\left(r_{2}-1\right) \tilde{\nu}_{\tilde{\mathcal{P}}_{1}}^{L}\left(z_{1}\right)-$ $\tilde{v}_{\tilde{\mathcal{P}}_{1}}^{L}\left(z_{1}\right) \wedge \tilde{v}_{\tilde{\mathcal{P}}_{2}}^{L}\left(z_{2}\right)$.

for all $\left(z_{1}, z_{2}\right) \in V_{1} \times V_{2}$.

Proof For any vertex $\left(z_{1}, z_{2}\right) \in V_{1} \times V_{2}$,

(i) If $\tilde{\mu}_{\tilde{\mathcal{P}}_{1}}^{L} \geq \tilde{\mu}_{\tilde{\mathcal{Q}}_{2}}^{L}, \tilde{\mu}_{\tilde{\mathcal{P}}_{2}}^{L} \geq \tilde{\mu}_{\tilde{\mathcal{Q}}_{1}}^{L}$ 


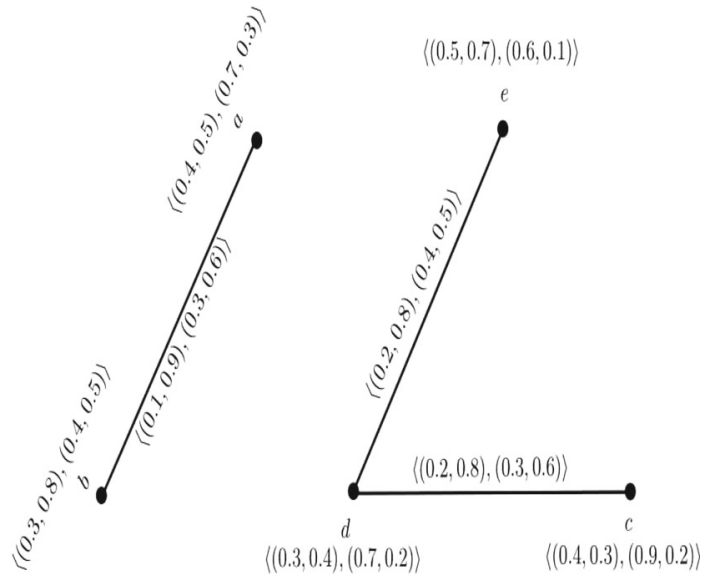

Fig. 6 SIVPFGs

$$
\begin{aligned}
& \left(t \mathrm{~d}_{\tilde{\mu}^{L}}\right)_{\tilde{\mathcal{G}}_{1}\left[\tilde{\mathcal{G}}_{2}\right]}\left(z_{1}, z_{2}\right) \\
& =\sum_{\left(y_{1}, y_{2}\right)\left(z_{1}, z_{2}\right) \in E_{1} \circ E_{2}}\left(\tilde{\mu}_{\tilde{\mathcal{Q}}_{1}}^{L} \circ \tilde{\mu}_{\tilde{\mathcal{Q}}_{2}}^{L}\right)\left(\left(y_{1}, y_{2}\right)\left(z_{1}, z_{2}\right)\right) \\
& +\left(\tilde{\mu}_{\tilde{\mathcal{P}}_{1}}^{L} \circ \tilde{\mu}_{\tilde{\mathcal{P}}_{2}}^{L}\right)\left(z_{1}, z_{2}\right) \\
& =\sum_{y_{1}=z_{1}, y_{2} z_{2} \in E_{2}} \tilde{\mu}_{\tilde{\mathcal{P}}_{1}}^{L}\left(z_{1}\right) \wedge \tilde{\mu}_{\tilde{\mathcal{Q}}_{2}}^{L}\left(y_{2} z_{2}\right) \\
& +\sum_{y_{2}=z_{2}, y_{1} z_{1} \in E_{1}} \tilde{\mu}_{\tilde{\mathcal{P}}_{2}}^{L}\left(z_{2}\right) \wedge \tilde{\mu}_{\tilde{\mathcal{Q}}_{1}}^{L}\left(y_{1} z_{1}\right) \\
& +\sum_{z_{2} \neq y_{2}, y_{1} z_{1} \in E_{1}} \tilde{\mu}_{\tilde{\mathcal{P}}_{2}}^{L}\left(y_{2}\right) \wedge \tilde{\mu}_{\tilde{\mathcal{P}}_{2}}^{L}\left(z_{2}\right) \wedge \tilde{\mu}_{\tilde{\mathcal{Q}}_{1}}^{L}\left(y_{1} z_{1}\right) \\
& +\tilde{\mu}_{\tilde{\mathcal{P}}_{1}}^{L}\left(z_{1}\right) \wedge \tilde{\mu}_{\tilde{\mathcal{P}}_{2}}^{L}\left(z_{2}\right) \\
& =\sum_{y_{2} z_{2} \in E_{2}} \tilde{\mu}_{\tilde{\mathcal{Q}}_{2}}^{L}\left(y_{2} z_{2}\right)+\sum_{y_{1} z_{1} \in E_{1}} \tilde{\mu}_{\tilde{\mathcal{Q}}_{1}}^{L}\left(y_{1} z_{1}\right) \\
& +\sum_{y_{1} z_{1} \in E_{1}} \tilde{\mu}_{\tilde{\mathcal{Q}}_{1}}^{L}\left(y_{1} z_{1}\right) \\
& +\tilde{\mu}_{\tilde{\mathcal{P}}_{1}}^{L}\left(z_{1}\right)+\tilde{\mu}_{\tilde{\mathcal{P}}_{2}}^{L}\left(z_{2}\right)-\tilde{\mu}_{\tilde{\mathcal{P}}_{1}}^{L}\left(z_{1}\right) \vee \tilde{\mu}_{\tilde{\mathcal{P}}_{2}}^{L}\left(z_{2}\right) \\
& =\left(t \mathrm{~d}_{\tilde{\mu}^{L}}\right)_{\tilde{\mathcal{G}}_{2}}\left(z_{2}\right)+r_{2}\left(t \mathrm{~d}_{\tilde{\mu}^{L}}\right) \tilde{\mathcal{G}}_{1}\left(z_{1}\right)-\left(r_{2}-1\right) \tilde{\mu}_{\tilde{\mathcal{P}}_{1}}^{L}\left(z_{1}\right) \\
& -\tilde{\mu}_{\tilde{\mathcal{P}}_{1}}^{L}\left(z_{1}\right) \vee \tilde{\mu}_{\tilde{\mathcal{P}}_{2}}^{L}\left(z_{2}\right) \text {. }
\end{aligned}
$$

Analogously, we can prove (ii)-(iv).

Example 4 Consider two SIVPFGs $\tilde{\mathcal{G}}_{1}$ and $\tilde{\mathcal{G}}_{2}$ on $V_{1}=\{a, b\}$ and $V_{2}=\{c, d, e\}$, respectively, as shown in Fig. 6. Then their lexicographic product $\tilde{\mathcal{G}}_{1}\left[\tilde{\mathcal{G}}_{2}\right]$ is shown in Fig. 7.

Since $\tilde{\mu}_{\tilde{\mathcal{P}}_{1}}^{L} \geq \tilde{\mu}_{\tilde{\mathcal{Q}}_{2}}^{L}, \tilde{v}_{\tilde{\mathcal{P}}_{1}}^{U} \leq \tilde{v}_{\tilde{\mathcal{Q}}_{2}}^{U}, \tilde{\mu}_{\tilde{\mathcal{P}}_{1}}^{U} \geq \tilde{\mu}_{\tilde{\mathcal{Q}}_{2}}^{U}, \tilde{v}_{\tilde{\mathcal{P}}_{1}}^{L} \leq \tilde{v}_{\tilde{\mathcal{Q}}_{2}}^{L}$ and $\tilde{\mu}_{\tilde{\mathcal{P}}_{2}}^{L} \geq \tilde{\mu}_{\tilde{\mathcal{Q}}_{1}}^{L}, \tilde{v}_{\tilde{\mathcal{P}}_{2}}^{U} \leq \tilde{v}_{\tilde{\mathcal{Q}}_{1}}^{U}, \tilde{\mu}_{\tilde{\mathcal{P}}_{2}}^{U} \geq \tilde{\mu}_{\tilde{\mathcal{Q}}_{1}}^{U}, \tilde{v}_{\tilde{\mathcal{P}}_{2}}^{L} \leq \tilde{v}_{\tilde{\mathcal{Q}}_{1}}^{L}$.

So, by Theorem 10, we must have

$$
\begin{aligned}
\left(\mathrm{d}_{\tilde{\mu}^{L}}\right)_{\tilde{\mathcal{G}}_{1}\left[\tilde{\mathcal{G}}_{2}\right]}(a, d) & =r_{2}\left(\mathrm{~d}_{\tilde{\mu}^{L}}\right)_{\tilde{\mathcal{G}}_{1}}(a)+\left(\mathrm{d}_{\tilde{\mu}^{L}}\right)_{\tilde{\mathcal{G}}_{2}}(d) \\
& =3(0.1)+0.4=0.7
\end{aligned}
$$

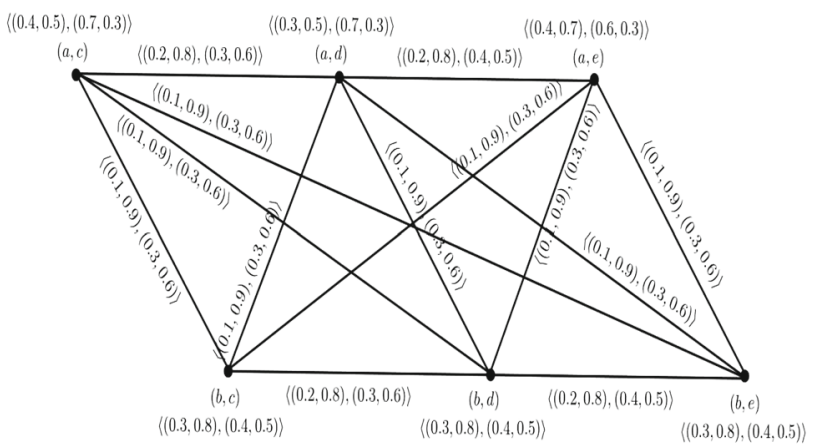

Fig. 7 The lexicographic product of two SIVPFGs

$$
\begin{aligned}
\left(\mathrm{d}_{\tilde{v} U}\right)_{\tilde{\mathcal{G}}_{1}\left[\tilde{\mathcal{G}}_{2}\right]}(a, d) & =r_{2}\left(\mathrm{~d}_{\tilde{v}^{U}}\right)_{\tilde{\mathcal{G}}_{1}}(a)+\left(\mathrm{d}_{\tilde{v} U}\right)_{\tilde{\mathcal{G}}_{2}}(d) \\
& =3(0.9)+1.6=4.3, \\
\left(\mathrm{~d}_{\tilde{\mu}^{U}}\right)_{\tilde{\mathcal{G}}_{1}\left[\tilde{\mathcal{G}}_{2}\right]}(a, d) & =r_{2}\left(\mathrm{~d}_{\tilde{\mu}^{U}}\right)_{\tilde{\mathcal{G}}_{1}}(a)+\left(\mathrm{d}_{\tilde{\mu}^{U}}\right)_{\tilde{\mathcal{G}}_{2}}(d) \\
& =3(0.3)+0.7=1.6, \\
\left(\mathrm{~d}_{\tilde{v} L}\right)_{\tilde{\mathcal{G}}_{1}\left[\tilde{\mathcal{G}}_{2}\right]}(a, d) & =r_{2}\left(\mathrm{~d}_{\left.\tilde{v}^{L}\right)} \tilde{\mathcal{G}}_{1}(a)+\left(\mathrm{d}_{\tilde{v}^{L}}\right)_{\tilde{\mathcal{G}}_{2}}(d)\right. \\
& =3(0.6)+1.1=2.9 .
\end{aligned}
$$

Therefore, $\mathrm{d}_{\tilde{\mathcal{G}}_{1}\left[\tilde{\mathcal{G}}_{2}\right]}(a, d)=\langle(0.7,1.6),(2.9,4.3)\rangle$. In addition, by Theorem 11, we have

$$
\begin{aligned}
& \left(t \mathrm{~d}_{\tilde{\mu}^{L}}\right)_{\tilde{\mathcal{G}}_{1}\left[\tilde{\mathcal{G}}_{2}\right]}(a, d) \\
& =r_{2}\left(t \mathrm{~d}_{\tilde{\mu}^{L}}\right)_{\tilde{\mathcal{G}}_{1}}(a)+\left(t \mathrm{~d}_{\tilde{\mu}^{L}}\right)_{\tilde{\mathcal{G}}_{2}}(d)-\left(r_{2}-1\right) \tilde{\mu}_{\tilde{\mathcal{P}}_{1}}^{L}(a) \\
& -\tilde{\mu}_{\tilde{\mathcal{P}}_{1}}^{L}(a) \vee \tilde{\mu}_{\tilde{\mathcal{P}}_{2}}^{L}(d) \\
& =3(0.5)+0.7-(3-1)(0.4)-0.4=1.0, \\
& \left(t \mathrm{~d}_{\tilde{v}^{U}}\right)_{\tilde{\mathcal{G}}_{1}\left[\tilde{\mathcal{G}}_{2}\right]}(a, d) \\
& =r_{2}\left(t \mathrm{~d}_{\tilde{\nu} U}\right)_{\tilde{\mathcal{G}}_{1}}(a)+\left(t \mathrm{~d}_{\tilde{v}^{U}}\right) \tilde{\mathcal{G}}_{2}(d)-\left(r_{2}-1\right) \tilde{v}_{\tilde{\mathcal{P}}_{1}}^{U}(a) \\
& -\tilde{v}_{\tilde{\mathcal{P}}_{1}}^{U}(a) \wedge \tilde{v}_{\tilde{\mathcal{P}}_{2}}^{U}(d) \\
& =3(1.4)+2.0-(3-1)(0.5)-0.4=4.8 \text {, } \\
& \left(t \mathrm{~d}_{\tilde{\mu}^{U}}\right)_{\tilde{\mathcal{G}}_{1}\left[\tilde{\mathcal{G}}_{2}\right]}(a, d) \\
& =r_{2}\left(t \mathrm{~d}_{\tilde{\mu}^{U}}\right)_{\tilde{\mathcal{G}}_{1}}(a)+\left(t \mathrm{~d}_{\tilde{\mu}^{U}}\right)_{\tilde{\mathcal{G}}_{2}}(d)-\left(r_{2}-1\right) \tilde{\mu}_{\tilde{\mathcal{P}}_{1}}^{U}(a) \\
& -\tilde{\mu}_{\tilde{\mathcal{P}}_{1}}^{U}(a) \vee \tilde{\mu}_{\tilde{\mathcal{P}}_{2}}^{U}(d) \\
& =3(1.0)+1.4-(3-1)(0.7)-0.7=2.3 \text {, } \\
& \left(t \mathrm{~d}_{\tilde{v} L}\right)_{\tilde{\mathcal{G}}_{1}\left[\tilde{\mathcal{G}}_{2}\right]}(a, d) \\
& =r_{2}\left(t \mathrm{~d}_{\tilde{v}^{L}}\right)_{\tilde{\mathcal{G}}_{1}}(a) \\
& +\left(t \mathrm{~d}_{\tilde{v}^{L}}\right)_{\tilde{\mathcal{G}}_{2}}(d)-\left(r_{2}-1\right) \tilde{v}_{\tilde{\mathcal{P}}_{1}}^{L}(a)-\tilde{v}_{\tilde{\mathcal{P}}_{1}}^{L}(a) \wedge \tilde{v}_{\tilde{\mathcal{P}}_{2}}^{L}(d) \\
& =3(0.9)+1.3-(3-1)(0.3)-0.3=3.2 \text {. }
\end{aligned}
$$

Therefore, $\mathrm{d}_{\tilde{\mathcal{G}}_{1}\left[\tilde{\mathcal{G}}_{2}\right]}(a, d)=\langle(1.0,4.8),(2.3,3.2)\rangle$.

Similarly, we can find the degree and total degree of all the vertices in $\tilde{\mathcal{G}}_{1}\left[\tilde{\mathcal{G}}_{2}\right]$. 
Definition 23 Let $\tilde{\mathcal{G}}_{1}$ and $\tilde{\mathcal{G}}_{2}$ be two SIVPFGs of $G_{1}$ and $G_{2}$, respectively. The symmetric difference of $\tilde{\mathcal{G}}_{1}$ and $\tilde{\mathcal{G}}_{2}$ is denoted by $\tilde{\mathcal{G}}_{1} \oplus \tilde{\mathcal{G}}_{2}=\left(\tilde{\mathcal{P}}_{1} \oplus \tilde{\mathcal{P}}_{2}, \tilde{\mathcal{Q}}_{1} \oplus \tilde{\mathcal{Q}}_{2}\right)$ and defined as:

(i)

$\left\{\begin{array}{l}\left(\tilde{\mu}_{\tilde{\mathcal{P}}_{1}}^{L} \oplus \tilde{\mu}_{\tilde{\mathcal{P}}_{2}}^{L}\right)\left(z_{1}, z_{2}\right)=\tilde{\mu}_{\tilde{\mathcal{P}}_{1}}^{L}\left(z_{1}\right) \wedge \tilde{\mu}_{\tilde{\mathcal{P}}_{2}}^{L}\left(z_{2}\right) \\ \left(\tilde{v}_{\tilde{\mathcal{P}}_{1}}^{U} \oplus \tilde{v}_{\tilde{\mathcal{P}}_{2}}^{U}\right)\left(z_{1}, z_{2}\right)=\tilde{v}_{\tilde{\mathcal{P}}_{1}}^{U}\left(z_{1}\right) \vee \tilde{v}_{\tilde{\mathcal{P}}_{2}}^{U}\left(z_{2}\right) \\ \left(\tilde{\mu}_{\tilde{\mathcal{P}}_{1}}^{U} \oplus \tilde{\mu}_{\tilde{\mathcal{P}}_{2}}^{U}\right)\left(z_{1}, z_{2}\right)=\tilde{\mu}_{\tilde{\mathcal{P}}_{1}}^{U}\left(z_{1}\right) \wedge \tilde{\mu}_{\tilde{\mathcal{P}}_{2}}^{U}\left(z_{2}\right) \\ \left(\tilde{v}_{\tilde{\mathcal{P}}_{1}}^{L} \oplus \tilde{v}_{\tilde{\mathcal{P}}_{2}}^{L}\right)\left(z_{1}, z_{2}\right)=\tilde{v}_{\tilde{\mathcal{P}}_{1}}^{L}\left(z_{1}\right) \vee \tilde{v}_{\tilde{\mathcal{P}}_{2}}^{L}\left(z_{2}\right) \\ \quad \text { for all }\left(z_{1}, z_{2}\right) \in V_{1} \times V_{2},\end{array}\right.$

$\left\{\begin{array}{l}\left(\tilde{\mu}_{\tilde{\mathcal{Q}}_{1}}^{L} \oplus \tilde{\mu}_{\tilde{\mathcal{Q}}_{2}}^{L}\right)\left(\left(y, y_{2}\right)\left(y, z_{2}\right)\right)=\tilde{\mu}_{\tilde{\mathcal{P}}_{1}}^{L}(y) \wedge \tilde{\mu}_{\tilde{\mathcal{Q}}_{2}}^{L}\left(y_{2} z_{2}\right) \\ \left(\tilde{v}_{\tilde{\mathcal{Q}}_{1}}^{U} \oplus \tilde{v}_{\tilde{\mathcal{Q}}_{2}}^{U}\right)\left(\left(y, y_{2}\right)\left(y, z_{2}\right)\right)=\tilde{v}_{\tilde{\mathcal{P}}_{1}}^{U}(y) \vee \tilde{v}_{\tilde{\mathcal{Q}}_{2}}^{U}\left(y_{2} z_{2}\right) \\ \left(\tilde{\mu}_{\tilde{\mathcal{Q}}_{1}}^{U} \oplus \tilde{\mu}_{\tilde{\mathcal{Q}}_{2}}^{U}\right)\left(\left(y, y_{2}\right)\left(y, z_{2}\right)\right)=\tilde{\mu}_{\tilde{\mathcal{P}}_{1}}^{U}(y) \wedge \tilde{\mu}_{\tilde{\mathcal{Q}}_{2}}^{U}\left(y_{2} z_{2}\right) \\ \left(\tilde{v}_{\tilde{\mathcal{Q}}_{1}}^{L} \oplus \tilde{v}_{\tilde{\mathcal{Q}}_{2}}^{L}\right)\left(\left(y, y_{2}\right)\left(y, z_{2}\right)\right)=\tilde{v}_{\tilde{\mathcal{P}}_{1}}^{L}(y) \vee \tilde{v}_{\tilde{\mathcal{Q}}_{2}}^{L}\left(y_{2} z_{2}\right) \\ \quad \text { for all yeV } V_{1}, \text { for all } y_{2} z_{2} \in E_{2},\end{array}\right.$

(ii)

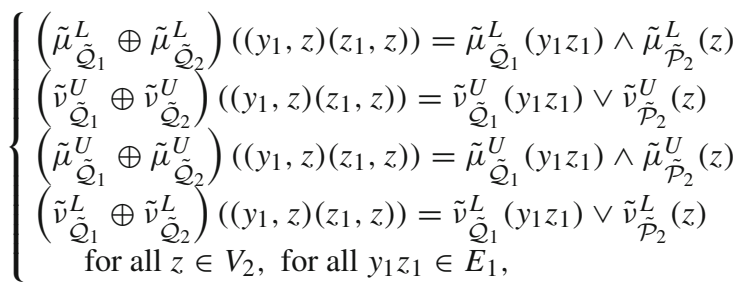

(iii)

$$
\begin{aligned}
& \int\left(\tilde{\mu}_{\tilde{\mathcal{Q}}_{1}}^{L} \oplus \tilde{\mu}_{\tilde{\mathcal{Q}}_{2}}^{L}\right)\left(\left(y_{1}, y_{2}\right)\left(z_{1}, z_{2}\right)\right) \\
& =\left\{\begin{array}{l}
\tilde{\mu}_{\tilde{\mathcal{P}}_{1}}^{L}\left(y_{1}\right) \wedge \tilde{\mu}_{\tilde{\mathcal{P}}_{1}}^{L}\left(z_{1}\right) \wedge \tilde{\mu}_{\tilde{\mathcal{Q}}_{2}}^{L}\left(y_{2} z_{2}\right) \\
\quad \text { for all } y_{1} z_{1} \notin E_{1}, \quad y_{2} z_{2} \in E_{2} \\
\text { or } \\
\tilde{\mu}_{\tilde{\mathcal{P}}_{2}}^{L}\left(y_{2}\right) \wedge \tilde{\mu}_{\tilde{\mathcal{P}}_{2}}^{L}\left(z_{2}\right) \wedge \tilde{\mu}_{\tilde{\mathcal{Q}}_{1}}^{L}\left(y_{1} z_{1}\right) \\
\quad \text { for all } y_{1} z_{1} \in E_{1}, y_{2} z_{2} \notin E_{2}
\end{array}\right. \\
& \left(\tilde{v}_{\tilde{\mathcal{Q}}_{1}}^{U} \oplus \tilde{v}_{\tilde{\mathcal{Q}}_{2}}^{U}\right)\left(\left(y_{1}, y_{2}\right)\left(z_{1}, z_{2}\right)\right) \\
& \left(\tilde{v}_{\tilde{\mathcal{P}}_{1}}^{U}\left(y_{1}\right) \vee \tilde{v}_{\tilde{\mathcal{P}}_{1}}^{U}\left(z_{1}\right) \vee \tilde{v}_{\tilde{\mathcal{Q}}_{2}}^{U}\left(y_{2} z_{2}\right)\right. \\
& =\left\{\begin{array}{l}
\text { for all } y_{1} z_{1} \notin E_{1}, y_{2} z_{2} \in E_{2} \\
\text { or }
\end{array}\right. \\
& \tilde{v}_{\tilde{\mathcal{P}}_{2}}^{U}\left(y_{2}\right) \vee \tilde{v}_{\tilde{\mathcal{P}}_{2}}^{U}\left(z_{2}\right) \vee \tilde{v}_{\tilde{\mathcal{Q}}_{1}}^{U}\left(y_{1} z_{1}\right) \\
& \begin{array}{l}
\left(\tilde{\mu}_{\tilde{\mathcal{Q}}_{1}}^{U} \oplus \tilde{\mu}_{\tilde{\mathcal{Q}}_{2}}^{U}\right)\left(\left(y_{1}, y_{2}\right)\left(z_{1}, z_{2}\right)\right) \\
=\left\{\begin{array}{l}
\tilde{\mu}_{\tilde{\mathcal{P}}_{1}}^{U}\left(y_{1}\right) \wedge \tilde{\mu}_{\tilde{\mathcal{P}}_{1}}^{U}\left(z_{1}\right) \wedge \tilde{\mu}_{\tilde{\mathcal{Q}}_{2}}^{U}\left(y_{2} z_{2}\right) \\
\quad \text { for all } y_{1} z_{1} \notin E_{1}, y_{2} z_{2} \in E_{2} \\
\text { or } \\
\tilde{\mu}_{\tilde{\mathcal{P}}_{2}}^{U}\left(y_{2}\right) \wedge \tilde{\mu}_{\tilde{\mathcal{P}}_{2}}^{U}\left(z_{2}\right) \wedge \tilde{\mu}_{\tilde{\mathcal{Q}}_{1}}^{U}\left(y_{1} z_{1}\right) \\
\quad \text { for all } y_{1} z_{1} \in E_{1}, y_{2} z_{2} \notin E_{2}
\end{array}\right.
\end{array} \\
& \left(\tilde{v}_{\tilde{\mathcal{Q}}_{1}}^{L} \oplus \tilde{v}_{\tilde{\mathcal{Q}}_{2}}^{L}\right)\left(\left(y_{1}, y_{2}\right)\left(z_{1}, z_{2}\right)\right) \\
& =\left\{\begin{array}{l}
\tilde{v}_{\tilde{\mathcal{P}}_{1}}^{L}\left(y_{1}\right) \vee \tilde{v}_{\tilde{\mathcal{P}}_{1}}^{L}\left(z_{1}\right) \vee \tilde{v}_{\tilde{\mathcal{Q}}_{2}}^{L}\left(y_{2} z_{2}\right) \\
\quad \text { for all } y_{1} z_{1} \notin E_{1}, y_{2} z_{2} \in E_{2} \\
\text { or } \\
\tilde{v}_{\tilde{\mathcal{P}}_{2}}^{L}\left(y_{2}\right) \vee \tilde{v}_{\tilde{\mathcal{P}}_{2}}^{L}\left(z_{2}\right) \vee \tilde{v}_{\tilde{\mathcal{Q}}_{1}}^{L}\left(y_{1} z_{1}\right) \\
\quad \text { for all } y_{1} z_{1} \in E_{1}, y_{2} z_{2} \notin E_{2} .
\end{array}\right.
\end{aligned}
$$

(iv)
Proposition 6 The symmetric difference $\tilde{\mathcal{G}}_{1} \oplus \tilde{\mathcal{G}}_{2}$ of two SIVPFGs $\tilde{\mathcal{G}}_{1}$ and $\tilde{\mathcal{G}}_{2}$ is a SIVPFG.

Definition 24 Let $\tilde{\mathcal{G}}_{1}$ and $\tilde{\mathcal{G}}_{2}$ be two SIVPFGs. For any vertex $\left(z_{1}, z_{2}\right) \in V_{1} \times V_{2}$,

$$
\begin{aligned}
& \left(\mathrm{d}_{\tilde{\mu}^{L}}\right)_{\tilde{\mathcal{G}}_{1} \oplus \tilde{\mathcal{G}}_{2}}\left(z_{1}, z_{2}\right) \\
& =\sum_{\left(y_{1}, y_{2}\right)\left(z_{1}, z_{2}\right) \in E_{1} \oplus E_{2}}\left(\tilde{\mu}_{\tilde{\mathcal{Q}}_{1}}^{L} \oplus \tilde{\mu}_{\tilde{\mathcal{Q}}_{2}}^{L}\right)\left(\left(y_{1}, y_{2}\right)\left(z_{1}, z_{2}\right)\right) \\
& =\sum_{y_{1}=z_{1}, y_{2} z_{2} \in E_{2}} \tilde{\mu}_{\tilde{\mathcal{P}}_{1}}^{L}\left(z_{1}\right) \wedge \tilde{\mu}_{\tilde{\mathcal{Q}}_{2}}^{L}\left(y_{2} z_{2}\right) \\
& \quad+\sum_{y_{2}=z_{2}, y_{1} z_{1} \in E_{1}} \tilde{\mu}_{\tilde{\mathcal{P}}_{2}}^{L}\left(z_{2}\right) \wedge \tilde{\mu}_{\tilde{\mathcal{Q}}_{1}}^{L}\left(y_{1} z_{1}\right) \\
& \quad+\sum_{y_{1} z_{1} \notin E_{1}, y_{2} z_{2} \in E_{2}} \tilde{\mu}_{\tilde{\mathcal{P}}_{1}}^{L}\left(z_{1}\right) \wedge \tilde{\mu}_{\tilde{\mathcal{P}}_{1}}^{L}\left(y_{1}\right) \wedge \tilde{\mu}_{\tilde{\mathcal{Q}}_{2}}^{L}\left(y_{2} z_{2}\right) \\
& \quad+\sum_{y_{1} z_{1} \in E_{1}, y_{2} z_{2} \notin E_{2}} \tilde{\mu}_{\tilde{\mathcal{P}}_{2}}^{L}\left(z_{2}\right) \wedge \tilde{\mu}_{\tilde{\mathcal{P}}_{2}}^{L}\left(y_{2}\right) \wedge \tilde{\mu}_{\tilde{\mathcal{Q}}_{1}}^{L}\left(y_{1} z_{1}\right),
\end{aligned}
$$$$
\left(\mathrm{d}_{\tilde{\nu} U}\right)_{\tilde{\mathcal{G}}_{1} \oplus \tilde{\mathcal{G}}_{2}}\left(z_{1}, z_{2}\right)
$$$$
=\sum_{\left(y_{1}, y_{2}\right)\left(z_{1}, z_{2}\right) \in E_{1} \oplus E_{2}}\left(\tilde{v}_{\tilde{\mathcal{Q}}_{1}}^{U} \oplus \tilde{v}_{\tilde{\mathcal{Q}}_{2}}^{U}\right)\left(\left(y_{1}, y_{2}\right)\left(z_{1}, z_{2}\right)\right)
$$$$
=\sum_{y_{1}=z_{1}, y_{2} z_{2} \in E_{2}} \tilde{v}_{\tilde{\mathcal{P}}_{1}}^{U}\left(z_{1}\right) \vee \tilde{v}_{\tilde{\mathcal{Q}}_{2}}^{U}\left(y_{2} z_{2}\right)
$$$$
+\sum_{y_{2}=z_{2}, y_{1} z_{1} \in E_{1}} \tilde{v}_{\tilde{\mathcal{P}}_{2}}^{U}\left(z_{2}\right) \vee \tilde{v}_{\tilde{\mathcal{Q}}_{1}}^{U}\left(y_{1} z_{1}\right)
$$$$
+\sum_{y_{1} z_{1} \notin E_{1}, y_{2} z_{2} \in E_{2}} \tilde{v}_{\tilde{\mathcal{P}}_{1}}^{U}\left(z_{1}\right) \vee \tilde{v}_{\tilde{\mathcal{P}}_{1}}^{U}\left(y_{1}\right) \vee \tilde{v}_{\tilde{\mathcal{Q}}_{2}}^{U}\left(y_{2} z_{2}\right)
$$$$
+\sum_{y_{1} z_{1} \in E_{1}, y_{2} z_{2} \notin E_{2}} \tilde{v}_{\tilde{\mathcal{P}}_{2}}^{U}\left(z_{2}\right) \vee \tilde{v}_{\tilde{\mathcal{P}}_{2}}^{U}\left(y_{2}\right) \vee \tilde{v}_{\tilde{\mathcal{Q}}_{1}}^{U}\left(y_{1} z_{1}\right),
$$$$
\left(\mathrm{d}_{\tilde{\mu}^{U}}\right)_{\tilde{\mathcal{G}}_{1} \oplus \tilde{\mathcal{G}}_{2}}\left(z_{1}, z_{2}\right)
$$$$
=\sum_{\left(y_{1}, y_{2}\right)\left(z_{1}, z_{2}\right) \in E_{1} \oplus E_{2}}\left(\tilde{\mu}_{\tilde{\mathcal{Q}}_{1}}^{U} \oplus \tilde{\mu}_{\tilde{\mathcal{Q}}_{2}}^{U}\right)\left(\left(y_{1}, y_{2}\right)\left(z_{1}, z_{2}\right)\right)
$$$$
=\sum_{y_{1}=z_{1}, y_{2} z_{2} \in E_{2}} \tilde{\mu}_{\tilde{\mathcal{P}}_{1}}^{U}\left(z_{1}\right) \wedge \tilde{\mu}_{\tilde{\mathcal{Q}}_{2}}^{U}\left(y_{2} z_{2}\right)
$$$$
+\sum_{y_{2}=z_{2}, y_{1} z_{1} \in E_{1}} \tilde{\mu}_{\tilde{\mathcal{P}}_{2}}^{U}\left(z_{2}\right) \wedge \tilde{\mu}_{\tilde{\mathcal{Q}}_{1}}^{U}\left(y_{1} z_{1}\right)
$$$$
+\sum_{y_{1} z_{1} \notin E_{1}, y_{2} z_{2} \in E_{2}} \tilde{\mu}_{\tilde{\mathcal{P}}_{1}}^{U}\left(z_{1}\right) \wedge \tilde{\mu}_{\tilde{\mathcal{P}}_{1}}^{U}\left(y_{1}\right) \wedge \tilde{\mu}_{\tilde{\mathcal{Q}}_{2}}^{U}\left(y_{2} z_{2}\right)
$$$$
+\sum_{y_{1} z_{1} \in E_{1}, y_{2} z_{2} \notin E_{2}} \tilde{\mu}_{\tilde{\mathcal{P}}_{2}}^{U}\left(z_{2}\right) \wedge \tilde{\mu}_{\tilde{\mathcal{P}}_{2}}^{U}\left(y_{2}\right) \wedge \tilde{\mu}_{\tilde{\mathcal{Q}}_{1}}^{U}\left(y_{1} z_{1}\right),
$$

$$
\begin{aligned}
& \left(\mathrm{d}_{\tilde{v}^{L}}\right)_{\tilde{\mathcal{G}}_{1} \oplus \tilde{\mathcal{G}}_{2}}\left(z_{1}, z_{2}\right) \\
& =\sum_{\left(y_{1}, y_{2}\right)\left(z_{1}, z_{2}\right) \in E_{1} \oplus E_{2}}\left(\tilde{v}_{\tilde{\mathcal{Q}}_{1}}^{L} \oplus \tilde{v}_{\tilde{\mathcal{Q}}_{2}}^{L}\right)\left(\left(y_{1}, y_{2}\right)\left(z_{1}, z_{2}\right)\right) \\
& =\sum_{y_{1}=z_{1}, y_{2} z_{2} \in E_{2}} \tilde{v}_{\tilde{\mathcal{P}}_{1}}^{L}\left(z_{1}\right) \vee \tilde{v}_{\tilde{\mathcal{Q}}_{2}}^{L}\left(y_{2} z_{2}\right)
\end{aligned}
$$




$$
\begin{aligned}
& +\sum_{y_{2}=z_{2}, y_{1} z_{1} \in E_{1}} \tilde{v}_{\tilde{\mathcal{P}}_{2}}^{L}\left(z_{2}\right) \vee \tilde{v}_{\tilde{\mathcal{Q}}_{1}}^{L}\left(y_{1} z_{1}\right) \\
& +\sum_{y_{1} z_{1} \notin E_{1}, y_{2} z_{2} \in E_{2}} \tilde{v}_{\tilde{\mathcal{P}}_{1}}^{L}\left(z_{1}\right) \vee \tilde{v}_{\tilde{\mathcal{P}}_{1}}^{L}\left(y_{1}\right) \vee \tilde{v}_{\tilde{\mathcal{Q}}_{2}}^{L}\left(y_{2} z_{2}\right) \\
& +\sum_{y_{1} z_{1} \in E_{1}, y_{2} z_{2} \notin E_{2}} \tilde{v}_{\tilde{\mathcal{P}}_{2}}^{L}\left(z_{2}\right) \vee \tilde{v}_{\tilde{\mathcal{P}}_{2}}^{L}\left(y_{2}\right) \vee \tilde{v}_{\tilde{\mathcal{Q}}_{1}}^{L}\left(y_{1} z_{1}\right) .
\end{aligned}
$$

Theorem 12 Let $\tilde{\mathcal{G}}_{1}$ and $\tilde{\mathcal{G}}_{2}$ be two SIVPFGs. If $\tilde{\mu}_{\tilde{\mathcal{P}}_{1}}^{L} \geq$ $\tilde{\mu}_{\tilde{\mathcal{Q}}_{2}}^{L}, \tilde{v}_{\tilde{\mathcal{P}}_{1}}^{U} \leq \tilde{v}_{\tilde{\mathcal{Q}}_{2}}^{U}, \tilde{\mu}_{\tilde{\mathcal{P}}_{1}}^{U} \geq \tilde{\mu}_{\tilde{\mathcal{Q}}_{2}}^{U}, \tilde{v}_{\tilde{\mathcal{P}}_{1}}^{L} \leq \tilde{v}_{\tilde{\mathcal{Q}}_{2}}^{L}$ and $\tilde{\mu}_{\tilde{\mathcal{P}}_{2}}^{L} \geq$ $\tilde{\mu}_{\tilde{\mathcal{Q}}_{1}}^{L}, \tilde{v}_{\tilde{\mathcal{P}}_{2}}^{U} \leq \tilde{v}_{\tilde{\mathcal{Q}}_{1}}^{U}, \tilde{\mu}_{\tilde{\mathcal{P}}_{2}}^{U} \geq \tilde{\mu}_{\tilde{\mathcal{Q}}_{1}}^{U}, \tilde{v}_{\tilde{\mathcal{P}}_{2}}^{L} \leq \tilde{v}_{\tilde{\mathcal{Q}}_{1}}^{L} \cdot$ Then $\mathrm{d}_{\tilde{\mathcal{G}}_{1} \oplus \tilde{\mathcal{G}}_{2}}\left(z_{1}, z_{2}\right)=\left(r_{2}-\mathrm{d}_{G_{2}}\left(z_{2}\right)\right) \mathrm{d}_{\tilde{\mathcal{G}}_{1}}\left(z_{1}\right)+\left(r_{1}-\mathrm{d}_{G_{1}}\left(z_{1}\right)\right)$ $\mathrm{d}_{\tilde{\mathcal{G}}_{2}}\left(z_{2}\right)$ for all $\left(z_{1}, z_{2}\right) \in V_{1} \times V_{2}$.

Proof By definition of vertex degree of $\tilde{\mathcal{G}}_{1} \oplus \tilde{\mathcal{G}}_{2}$, we have

$$
\begin{aligned}
& \left(\mathrm{d}_{\tilde{\mu}^{L}}\right)_{\tilde{\mathcal{G}}_{1} \oplus \tilde{\mathcal{G}}_{2}}\left(z_{1}, z_{2}\right) \\
& =\sum_{\left(y_{1}, y_{2}\right)\left(z_{1}, z_{2}\right) \in E_{1} \oplus E_{2}}\left(\tilde{\mu}_{\tilde{\mathcal{Q}}_{1}}^{L} \oplus \tilde{\mu}_{\tilde{\mathcal{Q}}_{2}}^{L}\right)\left(\left(y_{1}, y_{2}\right)\left(z_{1}, z_{2}\right)\right) \\
& =\sum_{y_{1}=z_{1}, y_{2} z_{2} \in E_{2}} \tilde{\mu}_{\tilde{\mathcal{P}}_{1}}^{L}\left(z_{1}\right) \wedge \tilde{\mu}_{\tilde{\mathcal{Q}}_{2}}^{L}\left(y_{2} z_{2}\right) \\
& +\sum_{y_{2}=z_{2}, y_{1} z_{1} \in E_{1}} \tilde{\mu}_{\tilde{\mathcal{P}}_{2}}^{L}\left(z_{2}\right) \wedge \tilde{\mu}_{\tilde{\mathcal{Q}}_{1}}^{L}\left(y_{1} z_{1}\right) \\
& +\sum_{y_{1} z_{1} \notin E_{1}, y_{2} z_{2} \in E_{2}} \tilde{\mu}_{\tilde{\mathcal{P}}_{1}}^{L}\left(z_{1}\right) \wedge \tilde{\mu}_{\tilde{\mathcal{P}}_{1}}^{L}\left(y_{1}\right) \wedge \tilde{\mu}_{\tilde{\mathcal{Q}}_{2}}^{L}\left(y_{2} z_{2}\right) \\
& +\sum_{y_{1} z_{1} \in E_{1}, y_{2} z_{2} \notin E_{2}} \tilde{\mu}_{\tilde{\mathcal{P}}_{2}}^{L}\left(z_{2}\right) \wedge \tilde{\mu}_{\tilde{\mathcal{P}}_{2}}^{L}\left(y_{2}\right) \wedge \tilde{\mu}_{\tilde{\mathcal{Q}}_{1}}^{L}\left(y_{1} z_{1}\right) \\
& =\sum_{y_{2} z_{2} \in E_{2}} \tilde{\mu}_{\tilde{\mathcal{Q}}_{2}}^{L}\left(y_{2} z_{2}\right) \\
& +\sum_{y_{1} z_{1} \in E_{1}} \tilde{\mu}_{\tilde{\mathcal{Q}}_{1}}^{L}\left(y_{1} z_{1}\right)+\sum_{y_{1} z_{1} \notin E_{1}, y_{2} z_{2} \in E_{2}} \tilde{\mu}_{\tilde{\mathcal{Q}}_{2}}^{L}\left(y_{2} z_{2}\right) \\
& +\sum_{y_{1} z_{1} \in E_{1}, y_{2} z_{2} \notin E_{2}} \tilde{\mu}_{\tilde{\mathcal{Q}}_{1}}^{L}\left(y_{1} z_{1}\right) \\
& \text { (using } \left.\tilde{\mu}_{\tilde{\mathcal{P}}_{1}}^{L} \geq \tilde{\mu}_{\tilde{\mathcal{Q}}_{2}}^{L} \text { and } \tilde{\mu}_{\tilde{\mathcal{P}}_{2}}^{L} \geq \tilde{\mu}_{\tilde{\mathcal{Q}}_{1}}^{L}\right) \\
& =\left(r_{2}-\mathrm{d}_{G_{2}}\left(z_{2}\right)\right)\left(\mathrm{d}_{\tilde{\mu}^{L}}\right)_{\tilde{\mathcal{G}}_{1}}\left(z_{1}\right)+\left(r_{1}-\mathrm{d}_{G_{1}}\left(z_{1}\right)\right)\left(\mathrm{d}_{\tilde{\mu}^{L}}\right)_{\tilde{\mathcal{G}}_{2}}\left(z_{2}\right) \text {, }
\end{aligned}
$$

Similarly, we can prove $\left(\mathrm{d}_{\tilde{v}^{U}}\right)_{\tilde{\mathcal{G}}_{1} \oplus \tilde{\mathcal{G}}_{2}}\left(z_{1}, z_{2}\right)=\left(r_{2}-\right.$ $\left.\mathrm{d}_{G_{2}}\left(z_{2}\right)\right)\left(\mathrm{d}_{\tilde{v} U}\right)_{\tilde{\mathcal{G}}_{1}}\left(z_{1}\right)+\left(r_{1}-\mathrm{d}_{G_{1}}\left(z_{1}\right)\right)\left(\mathrm{d}_{\tilde{v}} U\right) \tilde{\mathcal{G}}_{2}\left(z_{2}\right)$, $\left(\mathrm{d}_{\tilde{\mu}^{U}}\right)_{\tilde{\mathcal{G}}_{1} \oplus \tilde{\mathcal{G}}_{2}}\left(z_{1}, z_{2}\right)=\left(r_{2}-\mathrm{d}_{G_{2}}\left(z_{2}\right)\right)\left(\mathrm{d}_{\tilde{\mu}^{U}}\right) \tilde{\mathcal{G}}_{1}\left(z_{1}\right)+\left(r_{1}-\right.$ $\left.\mathrm{d}_{G_{1}}\left(z_{1}\right)\right)\left(\mathrm{d}_{\tilde{\mu}^{U}}\right)_{\tilde{\mathcal{G}}_{2}}\left(z_{2}\right),\left(\mathrm{d}_{\tilde{v}^{L}}\right)_{\tilde{\mathcal{G}}_{1} \oplus \tilde{\mathcal{G}}_{2}}\left(z_{1}, z_{2}\right)=\left(r_{2}-\mathrm{d}_{G_{2}}\left(z_{2}\right)\right)$ $\left(\mathrm{d}_{\tilde{v}^{L}}\right)_{\tilde{\mathcal{G}}_{1}}\left(z_{1}\right)+\left(r_{1}-\mathrm{d}_{G_{1}}\left(z_{1}\right)\right)\left(\mathrm{d}_{\tilde{v}^{L}}\right)_{\tilde{\mathcal{G}}_{2}}\left(z_{2}\right)$. Hence $\mathrm{d}_{\tilde{\mathcal{G}}_{1} \oplus \tilde{\mathcal{G}}_{2}}$ $\left(z_{1}, z_{2}\right)=\left(r_{2}-\mathrm{d}_{G_{2}}\left(z_{2}\right)\right) \mathrm{d}_{\tilde{\mathcal{G}}_{1}}\left(z_{1}\right)+\left(r_{1}-\mathrm{d}_{G_{1}}\left(z_{1}\right)\right) \mathrm{d}_{\tilde{\mathcal{G}}_{2}}\left(z_{2}\right)$.

Theorem 13 Let $\tilde{\mathcal{G}}_{1}$ and $\tilde{\mathcal{G}}_{2}$ be two SIVPFGs. If

(i) $\tilde{\mu}_{\tilde{\mathcal{P}}_{1}}^{L} \geq \tilde{\mu}_{\tilde{\mathcal{Q}}_{2}}^{L}$ and $\tilde{\mu}_{\tilde{\mathcal{P}}_{2}}^{L} \geq \tilde{\mu}_{\tilde{\mathcal{Q}}_{1}}^{L}$, then $\left(t \mathrm{~d}_{\tilde{\mu}^{L}}\right)_{\tilde{\mathcal{G}}_{1} \oplus \tilde{\mathcal{G}}_{2}}\left(z_{1}, z_{2}\right)$ $=s_{2}\left(t \mathrm{~d}_{\tilde{\mu}^{L}}\right)_{\tilde{\mathcal{G}}_{1}}\left(z_{1}\right)+s_{1}\left(t \mathrm{~d}_{\left.\tilde{\mu}^{L}\right)} \tilde{\mathcal{G}}_{2}\left(z_{2}\right)-\left(s_{2}-1\right) \tilde{\mu}_{\tilde{\mathcal{P}}_{1}}^{L}\left(z_{1}\right)-\right.$ $\left(s_{1}-1\right) \tilde{\mu}_{\tilde{\mathcal{P}}_{2}}^{L}\left(z_{2}\right)-\tilde{\mu}_{\tilde{\mathcal{P}}_{1}}^{L}\left(z_{1}\right) \vee \tilde{\mu}_{\tilde{\mathcal{P}}_{2}}^{L}\left(z_{2}\right)$; (ii) $\tilde{v}_{\tilde{\mathcal{P}}_{1}}^{U} \leq \tilde{v}_{\tilde{\mathcal{Q}}_{2}}^{U}$ and $\tilde{v}_{\tilde{\mathcal{P}}_{2}}^{U} \leq \tilde{v}_{\tilde{\mathcal{Q}}_{1}}^{U}$, then $\left(t \mathrm{~d}_{\tilde{v} U}\right)_{\tilde{\mathcal{G}}_{1} \oplus \tilde{\mathcal{G}}_{2}}\left(z_{1}, z_{2}\right)=$ $s_{2}\left(t \mathrm{~d}_{\tilde{v} U}\right)_{\tilde{\mathcal{G}}_{1}}\left(z_{1}\right)+s_{1}\left(t \mathrm{~d}_{\tilde{v}^{U}}\right)_{\tilde{\mathcal{G}}_{2}}\left(z_{2}\right)-\left(s_{2}-1\right) \tilde{v}_{\tilde{\mathcal{P}}_{1}}^{U}\left(z_{1}\right)-$ $\left(s_{1}-1\right) \tilde{v}_{\tilde{\mathcal{P}}_{2}}^{U}\left(z_{2}\right)-\tilde{v}_{\tilde{\mathcal{P}}_{1}}^{U}\left(z_{1}\right) \wedge \tilde{v}_{\tilde{\mathcal{P}}_{2}}^{U}\left(z_{2}\right)$;

(iii) $\tilde{\mu}_{\tilde{\mathcal{P}}_{1}}^{U} \geq \tilde{\mu}_{\tilde{\mathcal{Q}}_{2}}^{U}$ and $\tilde{\mu}_{\tilde{\mathcal{P}}_{2}}^{U} \geq \tilde{\mu}_{\tilde{\mathcal{Q}}_{1}}^{U}$, then $\left(t \mathrm{~d}_{\tilde{\mu}^{U}}\right)_{\tilde{\mathcal{G}}_{1} \oplus \tilde{\mathcal{G}}_{2}}\left(z_{1}, z_{2}\right)$ $=s_{2}\left(t \mathrm{~d}_{\tilde{\mu}^{U}}\right)_{\tilde{\mathcal{G}}_{1}}\left(z_{1}\right)+s_{1}\left(t \mathrm{~d}_{\tilde{\mu}^{U}}\right) \tilde{\mathcal{G}}_{2}\left(z_{2}\right)-\left(s_{2}-1\right) \tilde{\mu}_{\tilde{\mathcal{P}}_{1}}^{U}\left(z_{1}\right)-$ $\left(s_{1}-1\right) \tilde{\mu}_{\tilde{\mathcal{P}}_{2}}^{U}\left(z_{2}\right)-\tilde{\mu}_{\tilde{\mathcal{P}}_{1}}^{U}\left(z_{1}\right) \vee \tilde{\mu}_{\tilde{\mathcal{P}}_{2}}^{U}\left(z_{2}\right)$;

(iv) $\tilde{v}_{\tilde{\mathcal{P}}_{1}}^{L} \leq \tilde{v}_{\tilde{\mathcal{Q}}_{2}}^{L}$ and $\tilde{v}_{\tilde{\mathcal{P}}_{2}}^{L} \leq \tilde{v}_{\tilde{\mathcal{Q}}_{1}}^{L}$, then $\left(t \mathrm{~d}_{\tilde{v}^{L}}\right)_{\tilde{\mathcal{G}}_{1} \oplus \tilde{\mathcal{G}}_{2}}\left(z_{1}, z_{2}\right)=$ $s_{2}\left(t \mathrm{~d}_{\tilde{v}^{L}}\right)_{\tilde{\mathcal{G}}_{1}}\left(z_{1}\right)+s_{1}\left(t \mathrm{~d}_{\tilde{v}^{L}}\right)_{\tilde{\mathcal{G}}_{2}}\left(z_{2}\right)-\left(s_{2}-1\right) \tilde{v}_{\tilde{\mathcal{P}}_{1}}^{L}\left(z_{1}\right)-$ $\left(s_{1}-1\right) \tilde{v}_{\tilde{\mathcal{P}}_{2}}^{L}\left(z_{2}\right)-\tilde{v}_{\tilde{\mathcal{P}}_{1}}^{L}\left(z_{1}\right) \wedge \tilde{v}_{\tilde{\mathcal{P}}_{2}}^{L}\left(z_{2}\right)$

where $s_{1}=\left(r_{1}-\mathrm{d}_{G_{1}}\left(z_{1}\right)\right)$ and $s_{2}=\left(r_{2}-\mathrm{d}_{G_{2}}\left(z_{2}\right)\right)$ for all $\left(z_{1}, z_{2}\right) \in V_{1} \times V_{2}$.

$$
\begin{aligned}
& \text { Proof (i) If } \tilde{\mu}_{\tilde{\mathcal{P}}_{1}}^{L} \geq \tilde{\mu}_{\tilde{\mathcal{Q}}_{2}}^{L}, \tilde{\mu}_{\tilde{\mathcal{P}}_{2}}^{L} \geq \tilde{\mu}_{\tilde{\mathcal{Q}}_{1}}^{L} \\
& \left(t \mathrm{~d}_{\tilde{\mu}^{L}} \tilde{\mathcal{G}}_{1} \oplus \tilde{\mathcal{G}}_{2}\left(z_{1}, z_{2}\right)\right. \\
& =\sum_{\left(y_{1}, y_{2}\right)\left(z_{1}, z_{2}\right) \in E_{1} \oplus E_{2}}\left(\tilde{\mu}_{\tilde{\mathcal{Q}}_{1}}^{L} \oplus \tilde{\mu}_{\tilde{\mathcal{Q}}_{2}}^{L}\right)\left(\left(y_{1}, y_{2}\right)\left(z_{1}, z_{2}\right)\right) \\
& +\left(\tilde{\mu}_{\tilde{\mathcal{P}}_{1}}^{L} \oplus \tilde{\mu}_{\tilde{\mathcal{P}}_{2}}^{L}\right)\left(z_{1}, z_{2}\right) \\
& =\sum_{y_{1}=z_{1}, y_{2} z_{2} \in E_{2}} \tilde{\mu}_{\tilde{\mathcal{P}}_{1}}^{L}\left(z_{1}\right) \wedge \tilde{\mu}_{\tilde{\mathcal{Q}}_{2}}^{L}\left(y_{2} z_{2}\right) \\
& +\sum_{y_{2}=z_{2}, y_{1} z_{1} \in E_{1}} \tilde{\mu}_{\tilde{\mathcal{P}}_{2}}^{L}\left(z_{2}\right) \wedge \tilde{\mu}_{\tilde{\mathcal{Q}}_{1}}^{L}\left(y_{1} z_{1}\right) \\
& +\sum_{y_{1} z_{1} \notin E_{1}, y_{2} z_{2} \in E_{2}} \tilde{\mu}_{\tilde{\mathcal{P}}_{1}}^{L}\left(z_{1}\right) \wedge \tilde{\mu}_{\tilde{\mathcal{P}}_{1}}^{L}\left(y_{1}\right) \wedge \tilde{\mu}_{\tilde{\mathcal{Q}}_{2}}^{L}\left(y_{2} z_{2}\right) \\
& +\sum_{y_{1} z_{1} \in E_{1}, y_{2} z_{2} \notin E_{2}} \tilde{\mu}_{\tilde{\mathcal{P}}_{2}}^{L}\left(z_{2}\right) \wedge \tilde{\mu}_{\tilde{\mathcal{P}}_{2}}^{L}\left(y_{2}\right) \wedge \tilde{\mu}_{\tilde{\mathcal{Q}}_{1}}^{L}\left(y_{1} z_{1}\right) \\
& +\tilde{\mu}_{\tilde{\mathcal{P}}_{1}}^{L}\left(z_{1}\right) \wedge \tilde{\mu}_{\tilde{\mathcal{P}}_{2}}^{L}\left(z_{2}\right) \\
& =\sum_{y_{2} z_{2} \in E_{2}} \tilde{\mu}_{\tilde{\mathcal{Q}}_{2}}^{L}\left(y_{2} z_{2}\right) \\
& +\sum_{y_{1} z_{1} \in E_{1}} \tilde{\mu}_{\tilde{\mathcal{Q}}_{1}}^{L}\left(y_{1} z_{1}\right)+\sum_{y_{1} z_{1} \notin E_{1}, y_{2} z_{2} \in E_{2}} \tilde{\mu}_{\tilde{\mathcal{Q}}_{2}}^{L}\left(y_{2} z_{2}\right) \\
& +\sum_{y_{1} z_{1} \in E_{1}, y_{2} z_{2} \notin E_{2}} \tilde{\mu}_{\tilde{\mathcal{Q}}_{1}}^{L}\left(y_{1} z_{1}\right)+\tilde{\mu}_{\tilde{\mathcal{P}}_{2}}^{L}\left(z_{2}\right) \\
& +\tilde{\mu}_{\tilde{\mathcal{P}}_{1}}^{L}\left(z_{1}\right)-\tilde{\mu}_{\tilde{\mathcal{P}}_{1}}^{L}\left(z_{1}\right) \vee \tilde{\mu}_{\tilde{\mathcal{P}}_{2}}^{L}\left(z_{2}\right) \\
& =s_{2}\left(t \mathrm{~d}_{\tilde{\mu}^{L}}\right) \tilde{\mathcal{G}}_{1}\left(z_{1}\right)+s_{1}\left(t \mathrm{~d}_{\tilde{\mu}^{L}}\right) \tilde{\mathcal{G}}_{2}\left(z_{2}\right) \\
& -\left(s_{2}-1\right) \tilde{\mu}_{\tilde{\mathcal{P}}_{1}}^{L}\left(z_{1}\right)-\left(s_{1}-1\right) \tilde{\mu}_{\tilde{\mathcal{P}}_{2}}^{L}\left(z_{2}\right) \\
& -\tilde{\mu}_{\tilde{\mathcal{P}}_{1}}^{L}\left(z_{1}\right) \vee \tilde{\mu}_{\tilde{\mathcal{P}}_{2}}^{L}\left(z_{2}\right),
\end{aligned}
$$

Similarly, we can prove (ii), (iii) and (iv).

Example 5 Consider two SIVPFGs $\tilde{\mathcal{G}}_{1}$ and $\tilde{\mathcal{G}}_{2}$ as in Example 4, where $\tilde{\mu}_{\tilde{\mathcal{P}}_{1}}^{L} \geq \tilde{\mu}_{\tilde{\mathcal{Q}}_{2}}^{L}, \tilde{v}_{\tilde{\mathcal{P}}_{1}}^{U} \leq \tilde{v}_{\tilde{\mathcal{Q}}_{2}}^{U}, \tilde{\mu}_{\tilde{\mathcal{P}}_{1}}^{U} \geq \tilde{\mu}_{\tilde{\mathcal{Q}}_{2}}^{U}, \tilde{v}_{\tilde{\mathcal{P}}_{1}}^{L} \leq \tilde{v}_{\tilde{\mathcal{Q}}_{2}}^{L}$ and $\tilde{\mu}_{\tilde{\mathcal{P}}_{2}}^{L} \geq \tilde{\mu}_{\tilde{\mathcal{Q}}_{1}}^{L}, \tilde{v}_{\tilde{\mathcal{P}}_{2}}^{U} \leq \tilde{v}_{\tilde{\mathcal{Q}}_{1}}^{U}, \tilde{\mu}_{\tilde{\mathcal{P}}_{2}}^{U} \geq \tilde{\mu}_{\tilde{\mathcal{Q}}_{1}}^{U}, \tilde{\nu}_{\tilde{\mathcal{P}}_{2}}^{L} \leq \tilde{v}_{\tilde{\mathcal{Q}}_{1}}^{L}$ and their lexicographic product $\tilde{\mathcal{G}}_{1}\left[\tilde{\mathcal{G}}_{2}\right]$ is shown in Fig. 8. 


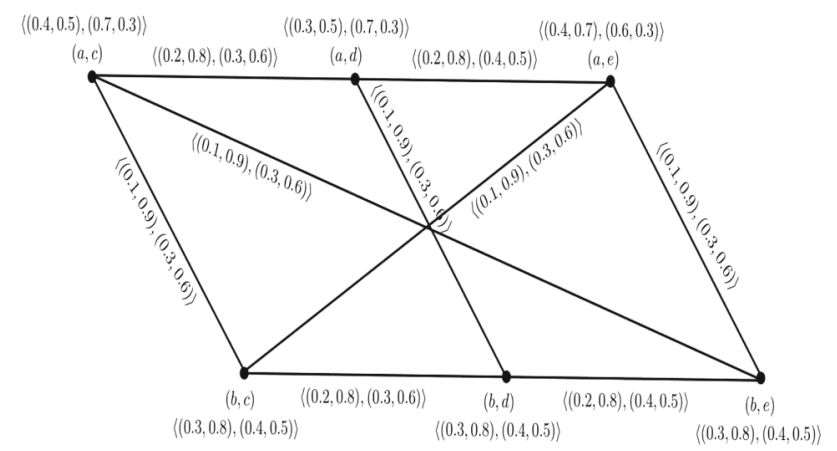

Fig. 8 The symmetric difference of two SIVPFGs

Then, by Theorem 12, we have

$$
\begin{aligned}
\left(\mathrm{d}_{\tilde{\mu}^{L}}\right)_{\tilde{\mathcal{G}}_{1} \oplus \tilde{\mathcal{G}}_{2}}(a, e)= & \left(s_{2}-\mathrm{d}_{G_{2}}(e)\right)\left(\mathrm{d}_{\tilde{\mu}^{L}}\right)_{\tilde{\mathcal{G}}_{1}}(a) \\
& +\left(s_{1}-\mathrm{d}_{G_{1}}(a)\right)\left(\mathrm{d}_{\tilde{\mu}^{L}} \tilde{\mathcal{G}}_{\tilde{\mathcal{G}}_{2}}(e)=0.4,\right. \\
\left(\mathrm{d}_{\tilde{v}^{U}}\right)_{\tilde{\mathcal{G}}_{1} \oplus \tilde{\mathcal{G}}_{2}}(a, e)= & \left(s_{2}-\mathrm{d}_{G_{2}}(e)\right)\left(\mathrm{d}_{\tilde{\mu}^{U}}\right)_{\tilde{\mathcal{G}}_{1}}(a) \\
& +\left(s_{1}-\mathrm{d}_{G_{1}}(a)\right)\left(\mathrm{d}_{\tilde{\mu}^{U}}\right)_{\tilde{\mathcal{G}}_{2}}(e)=2.6, \\
\left(\mathrm{~d}_{\tilde{\mu}^{U}}\right)_{\tilde{\mathcal{G}}_{1} \oplus \tilde{\mathcal{G}}_{2}}(a, e)= & \left(s_{2}-\mathrm{d}_{G_{2}}(e)\right)\left(\mathrm{d}_{\tilde{\mu}^{U}}\right)_{\tilde{\mathcal{G}}_{1}}(a) \\
& +\left(s_{1}-\mathrm{d}_{G_{1}}(a)\right)\left(\mathrm{d}_{\tilde{\mu}^{U}}\right)_{\tilde{\mathcal{G}}_{2}}(e)=1.0, \\
\left(\mathrm{~d}_{\tilde{v}^{L}}\right)_{\tilde{\mathcal{G}}_{1} \oplus \tilde{\mathcal{G}}_{2}}(a, e)= & \left(s_{2}-\mathrm{d}_{G_{2}}(e)\right)\left(\mathrm{d}_{\tilde{\mu}^{L}}\right)_{\tilde{\mathcal{G}}_{1}}(a) \\
& +\left(s_{1}-\mathrm{d}_{G_{1}}(a)\right)\left(\mathrm{d}_{\tilde{\mu}^{L}} \tilde{\mathcal{G}}_{\tilde{\mathcal{G}}_{2}}(e)=1.7 .\right.
\end{aligned}
$$

Therefore, $\mathrm{d}_{\tilde{\mathcal{G}}_{1} \oplus \tilde{\mathcal{G}}_{2}}(a, e)=\langle(0.4,2.6),(1.0,1.7)\rangle$.

In addition, by Theorem 13, we have

$$
\begin{aligned}
& \left(t \mathrm{~d}_{\tilde{\mu}^{L}}\right)_{\tilde{\mathcal{G}}_{1} \oplus \tilde{\mathcal{G}}_{2}}(a, e) \\
& =s_{2}\left(t \mathrm{~d}_{\tilde{\mu}^{L}}\right) \tilde{\mathcal{G}}_{1}(a)+s_{1}\left(t \mathrm{~d}_{\tilde{\mu}^{L}}\right) \tilde{\mathcal{G}}_{2}(e)-\left(s_{2}-1\right) \tilde{\mu}_{\tilde{\mathcal{P}}_{1}}^{L}(a) \\
& -\left(s_{1}-1\right) \tilde{\mu}_{\tilde{\mathcal{P}}_{2}}^{L}(e)-\tilde{\mu}_{\tilde{\mathcal{P}}_{1}}^{L}(a) \vee \tilde{\mu}_{\tilde{\mathcal{P}}_{2}}^{L}(e)=0.8, \\
& \left(t \mathrm{~d}_{\tilde{v} U}\right)_{\tilde{\mathcal{G}}_{1} \oplus \tilde{\mathcal{G}}_{2}}(a, e) \\
& =s_{2}\left(t \mathrm{~d}_{\tilde{\mu}^{U}}\right)_{\tilde{\mathcal{G}}_{1}}(a)+s_{1}\left(t \mathrm{~d}_{\tilde{\mu}^{U}}\right)_{\tilde{\mathcal{G}}_{2}}(e)-\left(s_{2}-1\right) \tilde{\mu}_{\tilde{\mathcal{P}}_{1}}^{U}(a) \\
& -\left(s_{1}-1\right) \tilde{\mu}_{\tilde{\mathcal{P}}_{2}}^{U}(e)-\tilde{\mu}_{\tilde{\mathcal{P}}_{1}}^{U}(a) \wedge \tilde{\mu}_{\tilde{\mathcal{P}}_{2}}^{U}(e)=3.3, \\
& \left(t \mathrm{~d}_{\tilde{\mu}^{U}}\right)_{\tilde{\mathcal{G}}_{1} \oplus \tilde{\mathcal{G}}_{2}}(a, e) \\
& =s_{2}\left(t \mathrm{~d}_{\tilde{\mu}^{U}}\right)_{\tilde{\mathcal{G}}_{1}}(a)+s_{1}\left(t \mathrm{~d}_{\tilde{\mu}^{U}}\right)_{\tilde{\mathcal{G}}_{2}}(e)-\left(s_{2}-1\right) \tilde{\mu}_{\tilde{\mathcal{P}}_{1}}^{U}(a) \\
& -\left(s_{1}-1\right) \tilde{\mu}_{\tilde{\mathcal{P}}_{2}}^{U}(e)-\tilde{\mu}_{\tilde{\mathcal{P}}_{1}}^{U}(a) \vee \tilde{\mu}_{\tilde{\mathcal{P}}_{2}}^{U}(e)=1.6, \\
& \left(t \mathrm{~d}_{\tilde{\nu}^{L}}\right)_{\tilde{\mathcal{G}}_{1} \oplus \tilde{\mathcal{G}}_{2}}(a, e) \\
& =s_{2}\left(t \mathrm{~d}_{\tilde{\mu}^{L}}\right)_{\tilde{\mathcal{G}}_{1}}(a)+s_{1}\left(t \mathrm{~d}_{\tilde{\mu}^{L}}\right)_{\tilde{\mathcal{G}}_{2}}(e)-\left(s_{2}-1\right) \tilde{\mu}_{\tilde{\mathcal{P}}_{1}}^{L}(a) \\
& -\left(s_{1}-1\right) \tilde{\mu}_{\tilde{\mathcal{P}}_{2}}^{L}(e)-\tilde{\mu}_{\tilde{\mathcal{P}}_{1}}^{L}(a) \wedge \tilde{\mu}_{\tilde{\mathcal{P}}_{2}}^{L}(e)=2.0 \text {. }
\end{aligned}
$$

Therefore, $t \mathrm{~d}_{\tilde{\mathcal{G}}_{1} \oplus \tilde{\mathcal{G}}_{2}}(a, e)=\langle(0.8,3.3),(1.6,2.0)\rangle$.

Similarly, we can find the degree and total degree of all the vertices in $\tilde{\mathcal{G}}_{1} \oplus \tilde{\mathcal{G}}_{2}$.
Definition 25 Let $\tilde{\mathcal{G}}_{1}$ and $\tilde{\mathcal{G}}_{2}$ be two SIVPFGs of $G_{1}$ and $G_{2}$, respectively. The disjunction of $\tilde{\mathcal{G}}_{1}$ and $\tilde{\mathcal{G}}_{2}$ is denoted by $\tilde{\mathcal{G}}_{1} \bigvee \tilde{\mathcal{G}}_{2}=\left(\tilde{\mathcal{P}}_{1} \bigvee \tilde{\mathcal{P}}_{2}, \tilde{\mathcal{Q}}_{1} \bigvee \tilde{\mathcal{Q}}_{2}\right)$ and defined as:

$$
\left\{\begin{array}{l}
\left(\tilde{\mu}_{\tilde{\mathcal{P}}_{1}}^{L} \vee \tilde{\mu}_{\tilde{\mathcal{P}}_{2}}^{L}\right)\left(z_{1}, z_{2}\right)=\tilde{\mu}_{\tilde{\mathcal{P}}_{1}}^{L}\left(z_{1}\right) \wedge \tilde{\mu}_{\tilde{\mathcal{P}}_{2}}^{L}\left(z_{2}\right) \\
\left(\tilde{v}_{\tilde{\mathcal{P}}_{1}}^{U} \vee \tilde{v}_{\tilde{\mathcal{P}}_{2}}^{U}\right)\left(z_{1}, z_{2}\right)=\tilde{v}_{\tilde{\mathcal{P}}_{1}}^{U}\left(z_{1}\right) \vee \tilde{v}_{\tilde{\mathcal{P}}_{2}}^{U}\left(z_{2}\right) \\
\left(\tilde{\mu}_{\tilde{\mathcal{P}}_{1}}^{U} \vee \tilde{\mu}_{\tilde{\mathcal{P}}_{2}}^{U}\right)\left(z_{1}, z_{2}\right)=\tilde{\mu}_{\tilde{\mathcal{P}}_{1}}^{U}\left(z_{1}\right) \wedge \tilde{\mu}_{\tilde{\mathcal{P}}_{2}}^{U}\left(z_{2}\right) \\
\left(\tilde{v}_{\tilde{\mathcal{P}}_{1}}^{L} \vee \tilde{v}_{\tilde{\mathcal{P}}_{2}}^{L}\right)\left(z_{1}, z_{2}\right)=\tilde{v}_{\tilde{\mathcal{P}}_{1}}^{L}\left(z_{1}\right) \vee \tilde{v}_{\tilde{\mathcal{P}}_{2}}^{L}\left(z_{2}\right) \\
\quad \text { for all }\left(z_{1}, z_{2}\right) \in V_{1} \times V_{2},
\end{array}\right.
$$

$$
\left\{\begin{array}{l}
\left(\tilde{\mu}_{\tilde{\mathcal{Q}}_{1}}^{L} \vee \tilde{\mu}_{\tilde{\mathcal{Q}}_{2}}^{L}\right)\left(\left(y, y_{2}\right)\left(y, z_{2}\right)\right) \\
=\tilde{\mu}_{\tilde{\mathcal{P}}_{1}}^{L}(y) \wedge \tilde{\mu}_{\tilde{\mathcal{Q}}_{2}}^{L}\left(y_{2} z_{2}\right) \\
\left(\tilde{v}_{\tilde{\mathcal{Q}}_{1}}^{U} \vee \tilde{v}_{\tilde{\mathcal{Q}}_{2}}^{U}\right)\left(\left(y, y_{2}\right)\left(y, z_{2}\right)\right) \\
=\tilde{v}_{\tilde{\mathcal{P}}_{1}}^{U}(y) \vee \tilde{v}_{\tilde{\mathcal{Q}}_{2}}^{U}\left(y_{2} z_{2}\right) \\
\left(\tilde{\mu}_{\tilde{\mathcal{Q}}_{1}}^{U} \vee \tilde{\mu}_{\tilde{\mathcal{Q}}_{2}}^{U}\right)\left(\left(y, y_{2}\right)\left(y, z_{2}\right)\right) \\
=\tilde{\mu}_{\tilde{\mathcal{P}}_{1}}^{U}(y) \wedge \tilde{\mu}_{\tilde{\mathcal{Q}}_{2}}^{U}\left(y_{2} z_{2}\right) \\
\left(\tilde{v}_{\tilde{\mathcal{Q}}_{1}}^{L} \bigvee \tilde{v}_{\tilde{\mathcal{Q}}_{2}}^{L}\right)\left(\left(y, y_{2}\right)\left(y, z_{2}\right)\right) \\
=\tilde{v}_{\tilde{\mathcal{P}}_{1}}^{L}(y) \vee \tilde{v}_{\tilde{\mathcal{Q}}_{2}}^{L}\left(y_{2} z_{2}\right) \\
\quad \text { for all } y \in V_{1}, \text { for all } y_{2} z_{2} \in E_{2},
\end{array}\right.
$$

$$
\text { (iii) }\left\{\begin{array}{c}
\left(\tilde{\mu}_{\tilde{\mathcal{Q}}_{1}}^{L} \vee \tilde{\mu}_{\tilde{\mathcal{Q}}_{2}}^{L}\right)\left(\left(y_{1}, z\right)\left(z_{1}, z\right)\right) \\
=\tilde{\mu}_{\tilde{\mathcal{Q}}_{1}}^{L}\left(y_{1} z_{1}\right) \wedge \tilde{\mu}_{\tilde{\mathcal{P}}_{2}}^{L}(z) \\
\left(\tilde{v}_{\tilde{\mathcal{Q}}_{1}}^{U} \vee \tilde{v}_{\tilde{\mathcal{Q}}_{2}}^{U}\right)\left(\left(y_{1}, z\right)\left(z_{1}, z\right)\right) \\
=\tilde{v}_{\tilde{\mathcal{Q}}_{1}}^{U}\left(y_{1} z_{1}\right) \vee \tilde{v}_{\tilde{\mathcal{P}}_{2}}^{U}(z) \\
\left(\tilde{\mu}_{\tilde{\mathcal{Q}}_{1}}^{U} \vee \tilde{\mu}_{\tilde{\mathcal{Q}}_{2}}^{U}\right)\left(\left(y_{1}, z\right)\left(z_{1}, z\right)\right) \\
=\tilde{\mu}_{\tilde{\mathcal{Q}}_{1}}^{U}\left(y_{1} z_{1}\right) \wedge \tilde{\mu}_{\tilde{\mathcal{P}}_{2}}^{U}(z) \\
\left(\tilde{v}_{\tilde{\mathcal{Q}}_{1}}^{L} \vee \tilde{v}_{\tilde{\mathcal{Q}}_{2}}^{L}\right)\left(\left(y_{1}, z\right)\left(z_{1}, z\right)\right) \\
=\tilde{v}_{\tilde{\mathcal{Q}}_{1}}^{L}\left(y_{1} z_{1}\right) \vee \tilde{v}_{\tilde{\mathcal{P}}_{2}}^{L}(z) \\
\quad \text { for all } z \in V_{2}, \text { for all } y_{1} z_{1} \in E_{1},
\end{array}\right.
$$

(iv) $\left\{\begin{array}{c}\left(\tilde{\mu}_{\tilde{\mathcal{Q}}_{1}}^{L} \vee \tilde{\mu}_{\tilde{\mathcal{Q}}_{2}}^{L}\right)\left(\left(y_{1}, y_{2}\right)\left(z_{1}, z_{2}\right)\right) \\ =\tilde{\mu}_{\tilde{\mathcal{P}}_{1}}^{L}\left(y_{1} z_{1}\right) \wedge \tilde{\mu}_{\tilde{\mathcal{Q}}_{2}}^{L}\left(y_{2} z_{2}\right) \\ \left(\tilde{v}_{\tilde{\mathcal{Q}}_{1}}^{U} \vee \tilde{v}_{\tilde{\mathcal{Q}}_{2}}^{U}\right)\left(\left(y_{1}, y_{2}\right)\left(z_{1}, z_{2}\right)\right) \\ =\tilde{v}_{\tilde{\mathcal{P}}_{1}}^{U}\left(y_{1} z_{1}\right) \vee \tilde{v}_{\tilde{\mathcal{Q}}_{2}}^{U}\left(y_{2} z_{2}\right) \\ \left(\tilde{\mu}_{\tilde{\mathcal{Q}}_{1}}^{U} \vee \tilde{\mu}_{\tilde{\mathcal{Q}}_{2}}^{U}\right)\left(\left(y_{1}, y_{2}\right)\left(z_{1}, z_{2}\right)\right) \\ =\tilde{\mu}_{\tilde{\mathcal{P}}_{1}}^{U}\left(y_{1} z_{1}\right) \wedge \tilde{\mu}_{\tilde{\mathcal{Q}}_{2}}^{U}\left(y_{2} z_{2}\right) \\ \left(\tilde{v}_{\tilde{\mathcal{Q}}_{1}}^{L} \vee \tilde{v}_{\tilde{\mathcal{Q}}_{2}}^{L}\right)\left(\left(y_{1}, y_{2}\right)\left(z_{1}, z_{2}\right)\right) \\ =\tilde{v}_{\tilde{\mathcal{P}}_{1}}^{L}\left(y_{1} z_{1}\right) \vee \tilde{v}_{\tilde{\mathcal{Q}}_{2}}^{L}\left(y_{2} z_{2}\right) \\ \quad \text { for all } y_{1} z_{1} \in E_{1}, \text { for all } y_{2} z_{2} \in E_{2} .\end{array}\right.$ 


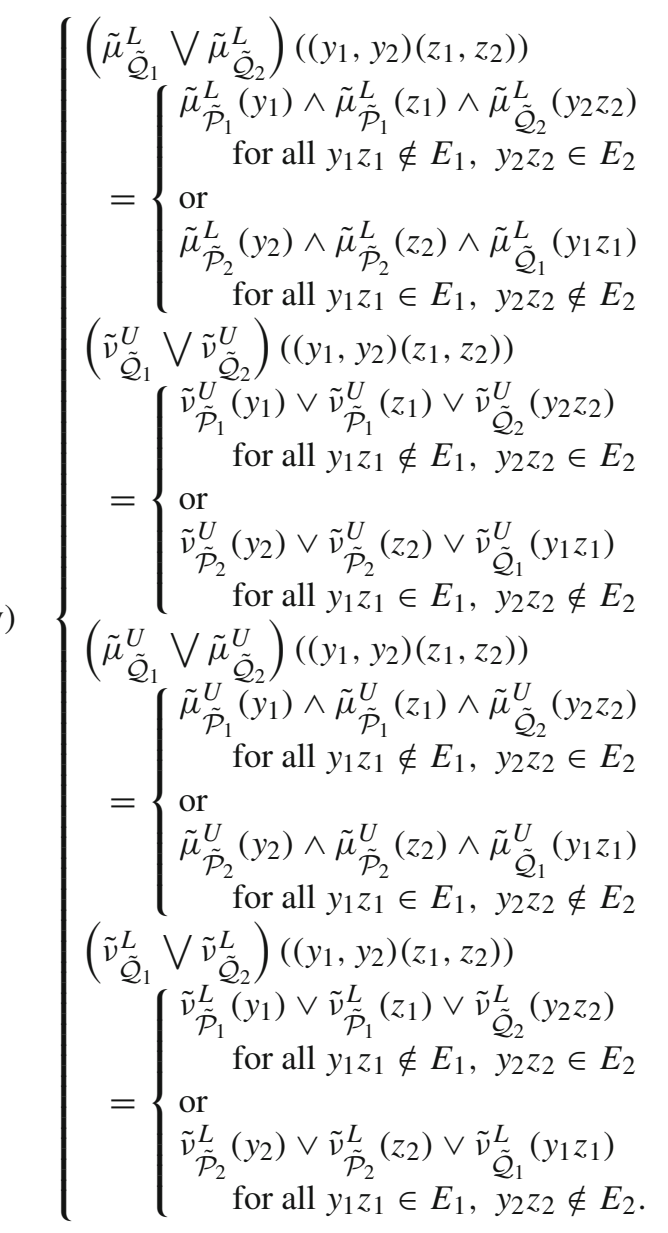

Proposition 7 The disjunction $\tilde{\mathcal{G}}_{1} \bigvee \tilde{\mathcal{G}}_{2}$ of two SIVPFGs $\tilde{\mathcal{G}}_{1}$ and $\tilde{\mathcal{G}}_{2}$ is a SIVPFG of $G_{1} \bigvee G_{2}$.

Definition 26 Let $\tilde{\mathcal{G}}_{1}$ and $\tilde{\mathcal{G}}_{2}$ be two SIVPFGs. For any vertex $\left(z_{1}, z_{2}\right) \in V_{1} \times V_{2}$,

$$
\begin{aligned}
& \left(\mathrm{d}_{\tilde{\mu}^{L}}^{L} \tilde{\mathcal{G}}_{1} \vee \tilde{\mathcal{G}}_{2}\left(z_{1}, z_{2}\right)\right. \\
& =\sum_{\left(y_{1}, y_{2}\right)\left(z_{1}, z_{2}\right) \in E_{1} \bigvee E_{2}}\left(\tilde{\mu}_{\tilde{\mathcal{Q}}_{1}}^{L} \bigvee \tilde{\mu}_{\tilde{\mathcal{Q}}_{2}}^{L}\right)\left(\left(y_{1}, y_{2}\right)\left(z_{1}, z_{2}\right)\right) \\
& =\sum_{y_{1}=z_{1}, y_{2} z_{2} \in E_{2}} \tilde{\mu}_{\tilde{\mathcal{P}}_{1}}^{L}\left(z_{1}\right) \wedge \tilde{\mu}_{\tilde{\mathcal{Q}}_{2}}^{L}\left(y_{2} z_{2}\right) \\
& \quad+\sum_{y_{2}=z_{2}, y_{1} z_{1} \in E_{1}} \tilde{\mu}_{\tilde{\mathcal{P}}_{2}}^{L}\left(z_{2}\right) \wedge \tilde{\mu}_{\tilde{\mathcal{Q}}_{1}}^{L}\left(y_{1} z_{1}\right) \\
& \quad+\sum_{y_{1} z_{1} \in E_{1}, y_{2} z_{2} \in E_{2}} \tilde{\mu}_{\tilde{\mathcal{Q}}_{1}}^{L}\left(y_{1} z_{1}\right) \wedge \tilde{\mu}_{\tilde{\mathcal{Q}}_{2}}^{L}\left(y_{2} z_{2}\right) \\
& \quad+\sum_{y_{1} z_{1} \notin E_{1}, y_{2} z_{2} \in E_{2}} \tilde{\mu}_{\tilde{\mathcal{P}}_{1}}^{L}\left(z_{1}\right) \wedge \tilde{\mu}_{\tilde{\mathcal{P}}_{1}}^{L}\left(y_{1}\right) \wedge \tilde{\mu}_{\tilde{\mathcal{Q}}_{2}}^{L}\left(y_{2} z_{2}\right) \\
& \quad+\sum_{y_{1} z_{1} \in E_{1}, y_{2} z_{2} \notin E_{2}} \tilde{\mu}_{\tilde{\mathcal{P}}_{2}}^{L}\left(z_{2}\right) \wedge \tilde{\mu}_{\tilde{\mathcal{P}}_{2}}^{L}\left(y_{2}\right) \wedge \tilde{\mu}_{\tilde{\mathcal{Q}}_{1}}^{L}\left(y_{1} z_{1}\right),
\end{aligned}
$$

$$
\begin{aligned}
& \left(\mathrm{d}_{\tilde{\nu}^{U}}\right)_{\tilde{\mathcal{G}}_{1} \bigvee \tilde{\mathcal{G}}_{2}}\left(z_{1}, z_{2}\right) \\
& =\sum_{\left(y_{1}, y_{2}\right)\left(z_{1}, z_{2}\right) \in E_{1} \bigvee E_{2}}\left(\tilde{v}_{\tilde{\mathcal{Q}}_{1}}^{U} \bigvee \tilde{v}_{\tilde{\mathcal{Q}}_{2}}^{U}\right)\left(\left(y_{1}, y_{2}\right)\left(z_{1}, z_{2}\right)\right) \\
& =\sum_{y_{1}=z_{1}, y_{2} z_{2} \in E_{2}} \tilde{v}_{\tilde{\mathcal{P}}_{1}}^{U}\left(z_{1}\right) \vee \tilde{v}_{\tilde{\mathcal{Q}}_{2}}^{U}\left(y_{2} z_{2}\right) \\
& +\sum_{y_{2}=z_{2}, y_{1} z_{1} \in E_{1}} \tilde{v}_{\tilde{\mathcal{P}}_{2}}^{U}\left(z_{2}\right) \vee \tilde{v}_{\tilde{\mathcal{Q}}_{1}}^{U}\left(y_{1} z_{1}\right) \\
& +\sum_{y_{1} z_{1} \in E_{1}, y_{2} z_{2} \in E_{2}} \tilde{v}_{\tilde{\mathcal{Q}}_{1}}^{U}\left(y_{1} z_{1}\right) \vee \tilde{v}_{\tilde{\mathcal{Q}}_{2}}^{U}\left(y_{2} z_{2}\right) \\
& +\sum_{y_{1} z_{1} \notin E_{1}, y_{2} z_{2} \in E_{2}} \tilde{v}_{\tilde{\mathcal{P}}_{1}}^{U}\left(z_{1}\right) \vee \tilde{v}_{\tilde{\mathcal{P}}_{1}}^{U}\left(y_{1}\right) \vee \tilde{v}_{\tilde{\mathcal{Q}}_{2}}^{U}\left(y_{2} z_{2}\right) \\
& +\sum_{y_{1} z_{1} \in E_{1}, y_{2} z_{2} \notin E_{2}} \tilde{v}_{\tilde{\mathcal{P}}_{2}}^{U}\left(z_{2}\right) \vee \tilde{v}_{\tilde{\mathcal{P}}_{2}}^{U}\left(y_{2}\right) \vee \tilde{v}_{\tilde{\mathcal{Q}}_{1}}^{U}\left(y_{1} z_{1}\right), \\
& \left(\mathrm{d}_{\tilde{\mu}^{U}} \tilde{\mathcal{G}}_{1} \bigvee \tilde{\mathcal{G}}_{2}\left(z_{1}, z_{2}\right)\right. \\
& =\sum_{\left(y_{1}, y_{2}\right)\left(z_{1}, z_{2}\right) \in E_{1} \bigvee E_{2}}\left(\tilde{\mu}_{\tilde{\mathcal{Q}}_{1}}^{U} \bigvee \tilde{\mu}_{\tilde{\mathcal{Q}}_{2}}^{U}\right)\left(\left(y_{1}, y_{2}\right)\left(z_{1}, z_{2}\right)\right) \\
& =\sum_{y_{1}=z_{1}, y_{2} z_{2} \in E_{2}} \tilde{\mu}_{\tilde{\mathcal{P}}_{1}}^{U}\left(z_{1}\right) \wedge \tilde{\mu}_{\tilde{\mathcal{Q}}_{2}}^{U}\left(y_{2} z_{2}\right) \\
& +\sum_{y_{2}=z_{2}, y_{1} z_{1} \in E_{1}} \tilde{\mu}_{\tilde{\mathcal{P}}_{2}}^{U}\left(z_{2}\right) \wedge \tilde{\mu}_{\tilde{\mathcal{Q}}_{1}}^{U}\left(y_{1} z_{1}\right) \\
& +\sum_{y_{1} z_{1} \in E_{1}, y_{2} z_{2} \in E_{2}} \tilde{\mu}_{\tilde{\mathcal{Q}}_{1}}^{U}\left(y_{1} z_{1}\right) \wedge \tilde{\mu}_{\tilde{\mathcal{Q}}_{2}}^{U}\left(y_{2} z_{2}\right) \\
& +\sum_{y_{1} z_{1} \notin E_{1}, y_{2} z_{2} \in E_{2}} \tilde{\mu}_{\tilde{\mathcal{P}}_{1}}^{U}\left(z_{1}\right) \wedge \tilde{\mu}_{\tilde{\mathcal{P}}_{1}}^{U}\left(y_{1}\right) \wedge \tilde{\mu}_{\tilde{\mathcal{Q}}_{2}}^{U}\left(y_{2} z_{2}\right) \\
& +\sum_{y_{1} z_{1} \in E_{1}, y_{2} z_{2} \notin E_{2}} \tilde{\mu}_{\tilde{\mathcal{P}}_{2}}^{U}\left(z_{2}\right) \wedge \tilde{\mu}_{\tilde{\mathcal{P}}_{2}}^{U}\left(y_{2}\right) \wedge \tilde{\mu}_{\tilde{\mathcal{Q}}_{1}}^{U}\left(y_{1} z_{1}\right),
\end{aligned}
$$

$$
\begin{aligned}
& \left(\mathrm{d}_{\tilde{v}^{L}}^{L} \tilde{\mathcal{G}}_{1} \vee \tilde{\mathcal{G}}_{2}\left(z_{1}, z_{2}\right)\right. \\
& =\sum_{\left(y_{1}, y_{2}\right)\left(z_{1}, z_{2}\right) \in E_{1} \bigvee E_{2}}\left(\tilde{v}_{\mathcal{Q}_{1}}^{L} \bigvee \tilde{v}_{\tilde{\mathcal{Q}}_{2}}^{L}\right)\left(\left(y_{1}, y_{2}\right)\left(z_{1}, z_{2}\right)\right) \\
& =\sum_{y_{1}=z_{1}, y_{2} z_{2} \in E_{2}} \tilde{v}_{\tilde{\mathcal{P}}_{1}}^{L}\left(z_{1}\right) \vee \tilde{v}_{\tilde{\mathcal{Q}}_{2}}^{L}\left(y_{2} z_{2}\right) \\
& \quad+\sum_{y_{2}=z_{2}, y_{1} z_{1} \in E_{1}} \tilde{v}_{\tilde{\mathcal{P}}_{2}}^{L}\left(z_{2}\right) \vee \tilde{v}_{\tilde{\mathcal{Q}}_{1}}^{L}\left(y_{1} z_{1}\right) \\
& \quad+\sum_{y_{1} z_{1} \in E_{1}, y_{2} z_{2} \in E_{2}} \tilde{v}_{\tilde{\mathcal{Q}}_{1}}^{L}\left(y_{1} z_{1}\right) \vee \tilde{v}_{\tilde{\mathcal{Q}}_{2}}^{L}\left(y_{2} z_{2}\right) \\
& \quad+\sum_{y_{1} z_{1} \notin E_{1}, y_{2} z_{2} \in E_{2}} \tilde{v}_{\tilde{\mathcal{P}}_{1}}^{L}\left(z_{1}\right) \vee \tilde{v}_{\tilde{\mathcal{P}}_{1}}^{L}\left(y_{1}\right) \vee \tilde{v}_{\tilde{\mathcal{Q}}_{2}}^{L}\left(y_{2} z_{2}\right) \\
& \quad \sum_{y_{1} z_{1} \in E_{1}, y_{2} z_{2} \notin E_{2}} \tilde{v}_{\tilde{\mathcal{P}}_{2}}^{L}\left(z_{2}\right) \vee \tilde{v}_{\tilde{\mathcal{P}}_{2}}^{L}\left(y_{2}\right) \vee \tilde{v}_{\tilde{\mathcal{Q}}_{1}}^{L}\left(y_{1} z_{1}\right) .
\end{aligned}
$$

Theorem 14 Let $\tilde{\mathcal{G}}_{1}$ and $\tilde{\mathcal{G}}_{2}$ be two SIVPFGs. If $\tilde{\mu}_{\tilde{\mathcal{P}}_{1}}^{L} \geq$ $\tilde{\mu}_{\tilde{\mathcal{Q}}_{2}}^{L}, \tilde{v}_{\tilde{\mathcal{P}}_{1}}^{U} \leq \tilde{v}_{\tilde{\mathcal{Q}}_{2}}^{U}, \tilde{\mu}_{\tilde{\mathcal{P}}_{1}}^{U} \geq \tilde{\mu}_{\tilde{\mathcal{Q}}_{2}}^{U}, \tilde{v}_{\tilde{\mathcal{P}}_{1}}^{L} \leq \tilde{v}_{\tilde{\mathcal{Q}}_{2}}^{L}, \tilde{\mu}_{\tilde{\mathcal{P}}_{2}}^{L} \geq$ 
$\tilde{\mu}_{\tilde{\mathcal{Q}}_{1}}^{L}, \tilde{v}_{\tilde{\mathcal{P}}_{2}}^{U} \leq \tilde{v}_{\tilde{\mathcal{Q}}_{1}}^{U}, \tilde{\mu}_{\tilde{\mathcal{P}}_{2}}^{U} \geq \tilde{\mu}_{\tilde{\mathcal{Q}}_{1}}^{U}, \tilde{v}_{\tilde{\mathcal{P}}_{2}}^{L} \leq \tilde{v}_{\tilde{\mathcal{Q}}_{1}}^{L}, \tilde{\mu}_{\tilde{\mathcal{Q}}_{1}}^{L} \leq$ $\tilde{\mu}_{\tilde{\mathcal{Q}}_{2}}^{L}, \tilde{v}_{\tilde{\mathcal{Q}}_{1}}^{U} \geq \tilde{v}_{\tilde{\mathcal{Q}}_{2}}^{U}, \tilde{\mu}_{\tilde{\mathcal{Q}}_{1}}^{U} \leq \tilde{\mu}_{\tilde{\mathcal{Q}}_{2}}^{U}, \tilde{v}_{\tilde{\mathcal{Q}}_{1}}^{L} \geq \tilde{v}_{\tilde{\mathcal{Q}}_{2}}^{L}$. Then $\mathrm{d}_{\tilde{\mathcal{G}}_{1} \bigvee \tilde{\mathcal{G}}_{2}}\left(z_{1}, z_{2}\right)=r_{2} \mathrm{~d}_{\tilde{\mathcal{G}}_{1}}\left(z_{1}\right)+r_{1} \mathrm{~d}_{\tilde{\mathcal{G}}_{2}}\left(z_{2}\right)-\mathrm{d}_{G_{1}}\left(z_{1}\right) \mathrm{d}_{\tilde{\mathcal{G}}_{2}}\left(z_{2}\right)$ for all $\left(z_{1}, z_{2}\right) \in V_{1} \times V_{2}$.

Proof By definition of vertex degree of $\tilde{\mathcal{G}}_{1} \bigvee \tilde{\mathcal{G}}_{2}$, we have

$$
\begin{aligned}
& \left(\mathrm{d}_{\tilde{\mu}^{L}} \tilde{\mathcal{G}}_{1} \bigvee \tilde{\mathcal{G}}_{2}\left(z_{1}, z_{2}\right)\right. \\
& =\sum_{\left(y_{1}, y_{2}\right)\left(z_{1}, z_{2}\right) \in E_{1} \bigvee E_{2}}\left(\tilde{\mu}_{\tilde{\mathcal{Q}}_{1}}^{L} \bigvee \tilde{\mu}_{\tilde{\mathcal{Q}}_{2}}^{L}\right)\left(\left(y_{1}, y_{2}\right)\left(z_{1}, z_{2}\right)\right) \\
& =\sum_{y_{1}=z_{1}, y_{2} z_{2} \in E_{2}} \tilde{\mu}_{\tilde{\mathcal{P}}_{1}}^{L}\left(z_{1}\right) \wedge \tilde{\mu}_{\tilde{\mathcal{Q}}_{2}}^{L}\left(y_{2} z_{2}\right) \\
& +\sum_{y_{2}=z_{2}, y_{1} z_{1} \in E_{1}} \tilde{\mu}_{\tilde{\mathcal{P}}_{2}}^{L}\left(z_{2}\right) \wedge \tilde{\mu}_{\tilde{\mathcal{Q}}_{1}}^{L}\left(y_{1} z_{1}\right) \\
& +\sum_{y_{1} z_{1} \in E_{1}, y_{2} z_{2} \in E_{2}} \tilde{\mu}_{\tilde{\mathcal{Q}}_{1}}^{L}\left(y_{1} z_{1}\right) \wedge \tilde{\mu}_{\tilde{\mathcal{Q}}_{2}}^{L}\left(y_{2} z_{2}\right) \\
& +\sum_{y_{1} z_{1} \notin E_{1}, y_{2} z_{2} \in E_{2}} \tilde{\mu}_{\tilde{\mathcal{P}}_{1}}^{L}\left(z_{1}\right) \wedge \tilde{\mu}_{\tilde{\mathcal{P}}_{1}}^{L}\left(y_{1}\right) \wedge \tilde{\mu}_{\tilde{\mathcal{Q}}_{2}}^{L}\left(y_{2} z_{2}\right) \\
& +\sum_{y_{1} z_{1} \in E_{1}, y_{2} z_{2} \notin E_{2}} \tilde{\mu}_{\tilde{\mathcal{P}}_{2}}^{L}\left(z_{2}\right) \wedge \tilde{\mu}_{\tilde{\mathcal{P}}_{2}}^{L}\left(y_{2}\right) \wedge \tilde{\mu}_{\tilde{\mathcal{Q}}_{1}}^{L}\left(y_{1} z_{1}\right) \\
& =\sum_{y_{2} z_{2} \in E_{2}} \tilde{\mu}_{\tilde{\mathcal{Q}}_{2}}^{L}\left(y_{2} z_{2}\right)+\sum_{y_{1} z_{1} \in E_{1}} \tilde{\mu}_{\tilde{\mathcal{Q}}_{1}}^{L}\left(y_{1} z_{1}\right) \\
& +\sum_{y_{1} z_{1} \in E_{1}} \tilde{\mu}_{\tilde{\mathcal{Q}}_{1}}^{L}\left(y_{1} z_{1}\right)+\sum_{y_{1} z_{1} \notin E_{1}, y_{2} z_{2} \in E_{2}} \tilde{\mu}_{\tilde{\mathcal{Q}}_{2}}^{L}\left(y_{2} z_{2}\right) \\
& +\sum_{y_{1} z_{1} \in E_{1}, y_{2} z_{2} \notin E_{2}} \tilde{\mu}_{\tilde{\mathcal{Q}}_{1}}^{L}\left(y_{1} z_{1}\right) \\
& \times\left(\text { using } \tilde{\mu}_{\tilde{\mathcal{P}}_{1}}^{L} \geq \tilde{\mu}_{\tilde{\mathcal{Q}}_{2}}^{L}, \tilde{\mu}_{\tilde{\mathcal{P}}_{2}}^{L} \geq \tilde{\mu}_{\tilde{\mathcal{Q}}_{1}}^{L} \text { and } \tilde{\mu}_{\tilde{\mathcal{Q}}_{1}}^{L} \leq \tilde{\mu}_{\tilde{\mathcal{Q}}_{2}}^{L}\right) \\
& =r_{2}\left(\mathrm{~d}_{\left.\tilde{\mu}^{L}\right)} \tilde{\mathcal{G}}_{1}\left(z_{1}\right)+r_{1}\left(\mathrm{~d}_{\tilde{\mu}^{L}}\right) \tilde{\mathcal{G}}_{2}\left(z_{2}\right)-\mathrm{d}_{G_{1}}\left(z_{1}\right)\left(\mathrm{d}_{\left.\tilde{\mu}^{L}\right)} \tilde{\mathcal{G}}_{2}\left(z_{2}\right)\right. \text {, }\right.
\end{aligned}
$$

Similarly, we can prove $\left(\mathrm{d}_{\tilde{\nu}} U\right) \tilde{\mathcal{G}}_{1} \bigvee \tilde{\mathcal{G}}_{2}\left(z_{1}, z_{2}\right)=r_{2}\left(\mathrm{~d}_{\tilde{\nu}^{U}}\right) \tilde{\mathcal{G}}_{1}\left(z_{1}\right)$ $+r_{1}\left(\mathrm{~d}_{\tilde{v}^{U}}\right)_{\tilde{\mathcal{G}}_{2}}\left(z_{2}\right)-\mathrm{d}_{G_{1}}\left(z_{1}\right)\left(\mathrm{d}_{\tilde{v}} U\right)_{\tilde{\mathcal{G}}_{2}}\left(z_{2}\right),\left(\mathrm{d}_{\tilde{\mu}^{U}}\right)_{\tilde{\mathcal{G}}_{1}} \bigvee \tilde{\mathcal{G}}_{2}\left(z_{1}, z_{2}\right)$ $=r_{2}\left(\mathrm{~d}_{\tilde{\mu}^{U}}\right)_{\tilde{\mathcal{G}}_{1}}\left(z_{1}\right)+r_{1}\left(\mathrm{~d}_{\tilde{\mu}^{U}}\right)_{\tilde{\mathcal{G}}_{2}}\left(z_{2}\right)-\mathrm{d}_{G_{1}}\left(z_{1}\right)\left(\mathrm{d}_{\tilde{\mu}^{U}}\right)_{\tilde{\mathcal{G}}_{2}}\left(z_{2}\right)$, $\left(\mathrm{d}_{\tilde{v}^{L}}\right)_{\tilde{\mathcal{G}}_{1} \bigvee \tilde{\mathcal{G}}_{2}}\left(z_{1}, z_{2}\right)=r_{2}\left(\mathrm{~d}_{\tilde{v}^{L}}\right) \tilde{\mathcal{G}}_{1}\left(z_{1}\right)+r_{1}\left(\mathrm{~d}_{\tilde{v}^{L}}\right)_{\tilde{\mathcal{G}}_{2}}\left(z_{2}\right)-$ $\mathrm{d}_{G_{1}}\left(z_{1}\right)\left(\mathrm{d}_{\tilde{v}^{L}}\right) \tilde{\mathcal{G}}_{2}\left(z_{2}\right)$. Hence $\mathrm{d}_{\tilde{\mathcal{G}}_{1}} \vee \tilde{\mathcal{G}}_{2}\left(z_{1}, z_{2}\right)=r_{2} \mathrm{~d}_{\tilde{\mathcal{G}}_{1}}\left(z_{1}\right)+$ $r_{1} \mathrm{~d}_{\tilde{\mathcal{G}}_{2}}\left(z_{2}\right)-\mathrm{d}_{G_{1}}\left(z_{1}\right) \mathrm{d}_{\tilde{\mathcal{G}}_{2}}\left(z_{2}\right)$.

\section{Theorem 15 Let $\tilde{\mathcal{G}}_{1}$ and $\tilde{\mathcal{G}}_{2}$ be two SIVPFGs. If}

(i) $\tilde{\mu}_{\tilde{\mathcal{P}}_{1}}^{L} \geq \tilde{\mu}_{\tilde{\mathcal{Q}}_{2}}^{L}, \tilde{\mu}_{\tilde{\mathcal{P}}_{2}}^{L} \geq \tilde{\mu}_{\tilde{\mathcal{Q}}_{1}}^{L}$ and $\tilde{\mu}_{\tilde{\mathcal{Q}}_{1}}^{L} \leq \tilde{\mu}_{\tilde{\mathcal{Q}}_{2}}^{L}$, then $\left(t \mathrm{~d}_{\tilde{\mu}^{L}}\right) \tilde{\mathcal{G}}_{1} \bigvee \tilde{\mathcal{G}}_{2}\left(z_{1}, z_{2}\right)=r_{2}\left(t \mathrm{~d}_{\tilde{\mu}^{L}}\right) \tilde{\mathcal{G}}_{1}\left(z_{1}\right)+r_{1}\left(t \mathrm{~d}_{\tilde{\mu}^{L}}\right) \tilde{\mathcal{G}}_{2}$ $\left(z_{2}\right)-\left(r_{2}-1\right) \tilde{\mu}_{\tilde{\mathcal{P}}_{1}}^{L}\left(z_{1}\right)-\left(r_{1}-1\right) \tilde{\mu}_{\tilde{\mathcal{P}}_{2}}^{L}\left(z_{2}\right)-\tilde{\mu}_{\tilde{\mathcal{P}}_{1}}^{L}\left(z_{1}\right) \vee$ $\tilde{\mu}_{\tilde{\mathcal{P}}_{2}}^{L}\left(z_{2}\right)$

(ii) $\tilde{v}_{\tilde{\mathcal{P}}_{1}}^{U} \leq \tilde{v}_{\tilde{\mathcal{Q}}_{2}}^{U}$, $\tilde{v}_{\tilde{\mathcal{P}}_{2}}^{U} \leq \tilde{v}_{\tilde{\mathcal{Q}}_{1}}^{U}$ and $\tilde{v}_{\tilde{\mathcal{Q}}_{1}}^{U} \geq \tilde{v}_{\tilde{\mathcal{Q}}_{2}}^{U}$, then $\left(t \mathrm{~d}_{\tilde{v} U}\right)_{\tilde{\mathcal{G}}_{1}} \bigvee \tilde{\mathcal{G}}_{2}\left(z_{1}, z_{2}\right)=r_{2}\left(t \mathrm{~d}_{\tilde{v}^{U}}\right)_{\tilde{\mathcal{G}}_{1}}\left(z_{1}\right)+r_{1}\left(t \mathrm{~d}_{\tilde{v}^{U}}\right)_{\tilde{\mathcal{G}}_{2}}$ $\left(z_{2}\right)-\left(r_{2}-1\right) \tilde{v}_{\tilde{\mathcal{P}}_{1}}^{U}\left(z_{1}\right)-\left(r_{1}-1\right) \tilde{v}_{\tilde{\mathcal{P}}_{2}}^{U}\left(z_{2}\right)-\tilde{v}_{\tilde{\mathcal{P}}_{1}}^{U}\left(z_{1}\right) \wedge$ $\tilde{v}_{\tilde{\mathcal{P}}_{2}}^{U}\left(z_{2}\right)$; (iii) $\tilde{\mu}_{\tilde{\mathcal{P}}_{1}}^{U} \geq \tilde{\mu}_{\tilde{\mathcal{Q}}_{2}}^{U}, \tilde{\mu}_{\tilde{\mathcal{P}}_{2}}^{U} \geq \tilde{\mu}_{\tilde{\mathcal{Q}}_{1}}^{U}$ and $\tilde{\mu}_{\tilde{\mathcal{Q}}_{1}}^{U} \leq \tilde{\mu}_{\tilde{\mathcal{Q}}_{2}}^{U}$, then $\left(t \mathrm{~d}_{\tilde{\mu}^{U}}\right)_{\tilde{\mathcal{G}}_{1} \bigvee \tilde{\mathcal{G}}_{2}}\left(z_{1}, z_{2}\right)=r_{2}\left(t \mathrm{~d}_{\tilde{\mu}^{U}}\right)_{\tilde{\mathcal{G}}_{1}}\left(z_{1}\right)+r_{1}\left(t \mathrm{~d}_{\tilde{\mu}^{U}}\right)_{\tilde{\mathcal{G}}_{2}}$ $\left(z_{2}\right)-\left(r_{2}-1\right) \tilde{\mu}_{\tilde{\mathcal{P}}_{1}}^{U}\left(z_{1}\right)-\left(r_{1}-1\right) \tilde{\mu}_{\tilde{\mathcal{P}}_{2}}^{U}\left(z_{2}\right)-\tilde{\mu}_{\tilde{\mathcal{P}}_{1}}^{U}\left(z_{1}\right) \vee$ $\tilde{\mu}_{\tilde{\mathcal{P}}_{2}}^{U}\left(z_{2}\right)$;

(iv) $\tilde{v}_{\tilde{\mathcal{P}}_{1}}^{L} \leq \tilde{v}_{\tilde{\mathcal{Q}}_{2}}^{L}, \tilde{v}_{\tilde{\mathcal{P}}_{2}}^{L} \leq \tilde{v}_{\tilde{\mathcal{Q}}_{1}}^{L}$ and $\tilde{v}_{\tilde{\mathcal{Q}}_{1}}^{L} \geq \tilde{v}_{\tilde{\mathcal{Q}}_{2}}^{L}$, then $\left(t \mathrm{~d}_{\tilde{v}^{L}}\right)_{\tilde{\mathcal{G}}_{1}} \bigvee \tilde{\mathcal{G}}_{2}\left(z_{1}, z_{2}\right)=r_{2}\left(t \mathrm{~d}_{\tilde{v}^{L}}\right)_{\tilde{\mathcal{G}}_{1}}\left(z_{1}\right)+r_{1}\left(t \mathrm{~d}_{\tilde{v}^{L}}\right)_{\tilde{\mathcal{G}}_{2}}$ $\left(z_{2}\right)-\left(r_{2}-1\right) \tilde{v}_{\tilde{\mathcal{P}}_{1}}^{L}\left(z_{1}\right)-\left(r_{1}-1\right) \tilde{v}_{\tilde{\mathcal{P}}_{2}}^{L}\left(z_{2}\right)-\tilde{v}_{\tilde{\mathcal{P}}_{1}}^{L}\left(z_{1}\right) \wedge$ $\tilde{v}_{\tilde{\mathcal{P}}_{2}}^{L}\left(z_{2}\right)$

for all $\left(z_{1}, z_{2}\right) \in V_{1} \times V_{2}$.

$$
\begin{aligned}
& \text { Proof (i) If } \tilde{\mu}_{\tilde{\mathcal{P}}_{1}}^{L} \geq \tilde{\mu}_{\tilde{\mathcal{Q}}_{2}}^{L} \text {, } \tilde{\mu}_{\tilde{\mathcal{P}}_{2}}^{L} \geq \tilde{\mu}_{\tilde{\mathcal{Q}}_{1}}^{L} \text { and } \tilde{\mu}_{\tilde{\mathcal{Q}}_{1}}^{L} \leq \tilde{\mu}_{\tilde{\mathcal{Q}}_{2}}^{L} \\
& \left(t \mathrm{~d}_{\tilde{\mu}^{L}} \tilde{\mathcal{G}}_{1} \bigvee \tilde{\mathcal{G}}_{2}\left(z_{1}, z_{2}\right)\right. \\
& =\sum_{\left(y_{1}, y_{2}\right)\left(z_{1}, z_{2}\right) \in E_{1} \bigvee E_{2}}\left(\tilde{\mu}_{\tilde{\mathcal{Q}}_{1}}^{L} \bigvee \tilde{\mu}_{\tilde{\mathcal{Q}}_{2}}^{L}\right)\left(\left(y_{1}, y_{2}\right)\left(z_{1}, z_{2}\right)\right) \\
& +\left(\tilde{\mu}_{\tilde{\mathcal{P}}_{1}}^{L} \bigvee \tilde{\mu}_{\tilde{\mathcal{P}}_{2}}^{L}\right)\left(z_{1}, z_{2}\right) \\
& =\sum_{y_{1}=z_{1}, y_{2} z_{2} \in E_{2}} \tilde{\mu}_{\tilde{\mathcal{P}}_{1}}^{L}\left(z_{1}\right) \wedge \tilde{\mu}_{\tilde{\mathcal{Q}}_{2}}^{L}\left(y_{2} z_{2}\right) \\
& +\sum_{y_{2}=z_{2}, y_{1} z_{1} \in E_{1}} \tilde{\mu}_{\tilde{\mathcal{P}}_{2}}^{L}\left(z_{2}\right) \wedge \tilde{\mu}_{\tilde{\mathcal{Q}}_{1}}^{L}\left(y_{1} z_{1}\right) \\
& +\sum_{y_{1} z_{1} \in E_{1}, y_{2} z_{2} \in E_{2}} \tilde{\mu}_{\tilde{\mathcal{Q}}_{1}}^{L}\left(y_{1} z_{1}\right) \wedge \tilde{\mu}_{\tilde{\mathcal{Q}}_{2}}^{L}\left(y_{2} z_{2}\right) \\
& +\sum_{y_{1} z_{1} \notin E_{1}, y_{2} z_{2} \in E_{2}} \tilde{\mu}_{\tilde{\mathcal{P}}_{1}}^{L}\left(z_{1}\right) \wedge \tilde{\mu}_{\tilde{\mathcal{P}}_{1}}^{L}\left(y_{1}\right) \wedge \tilde{\mu}_{\tilde{\mathcal{Q}}_{2}}^{L}\left(y_{2} z_{2}\right) \\
& +\sum_{y_{1} z_{1} \in E_{1}, y_{2} z_{2} \notin E_{2}} \tilde{\mu}_{\tilde{\mathcal{P}}_{2}}^{L}\left(z_{2}\right) \wedge \tilde{\mu}_{\tilde{\mathcal{P}}_{2}}^{L}\left(y_{2}\right) \wedge \tilde{\mu}_{\tilde{\mathcal{Q}}_{1}}^{L}\left(y_{1} z_{1}\right) \\
& +\tilde{\mu}_{\tilde{\mathcal{P}}_{1}}^{L}\left(z_{1}\right) \wedge \tilde{\mu}_{\tilde{\mathcal{P}}_{2}}^{L}\left(z_{2}\right) \\
& =\sum_{y_{2} z_{2} \in E_{2}} \tilde{\mu}_{\tilde{\mathcal{Q}}_{2}}^{L}\left(y_{2} z_{2}\right)+2 \sum_{y_{1} z_{1} \in E_{1}} \tilde{\mu}_{\tilde{\mathcal{Q}}_{1}}^{L}\left(y_{1} z_{1}\right) \\
& +\sum_{y_{1} z_{1} \notin E_{1}, y_{2} z_{2} \in E_{2}} \tilde{\mu}_{\tilde{\mathcal{Q}}_{2}}^{L}\left(y_{2} z_{2}\right) \\
& +\sum_{y_{1} z_{1} \in E_{1}, y_{2} z_{2} \notin E_{2}} \tilde{\mu}_{\tilde{\mathcal{Q}}_{1}}^{L}\left(y_{1} z_{1}\right)+\tilde{\mu}_{\tilde{\mathcal{P}}_{2}}^{L}\left(z_{2}\right)+\tilde{\mu}_{\tilde{\mathcal{P}}_{1}}^{L}\left(z_{1}\right) \\
& -\tilde{\mu}_{\tilde{\mathcal{P}}_{1}}^{L}\left(z_{1}\right) \vee \tilde{\mu}_{\tilde{\mathcal{P}}_{2}}^{L}\left(z_{2}\right) \\
& =r_{2}\left(t \mathrm{~d}_{\tilde{\mu}^{L}}\right)_{\tilde{\mathcal{G}}_{1}}\left(z_{1}\right)+r_{1}\left(t \mathrm{~d}_{\tilde{\mu}^{L}}\right)_{\tilde{\mathcal{G}}_{2}}\left(z_{2}\right) \\
& -\left(r_{2}-1\right) \tilde{\mu}_{\tilde{\mathcal{P}}_{1}}^{L}\left(z_{1}\right)-\left(r_{1}-1\right) \tilde{\mu}_{\tilde{\mathcal{P}}_{2}}^{L}\left(z_{2}\right) \\
& -\tilde{\mu}_{\tilde{\mathcal{P}}_{1}}^{L}\left(z_{1}\right) \vee \tilde{\mu}_{\tilde{\mathcal{P}}_{2}}^{L}\left(z_{2}\right) \text {. }
\end{aligned}
$$

Similarly, we can prove (ii), (iii) and (iv).

The degree of vertices in operations on SIVPFGs are given in Table 1. 
Table 1 The degree of vertices in operations on SIVPFGs

\begin{tabular}{lll}
\hline Operation & Notation & The degree $\mathrm{d}$ of a vertex $\left(z_{1}, z_{2}\right)$ in operations on SIVPFGs \\
\hline Tensor product & $\tilde{\mathcal{G}}_{1} \times \tilde{\mathcal{G}}_{2}$ & $\mathrm{~d}_{\tilde{\mathcal{G}}_{1} \times \tilde{\mathcal{G}}_{2}}\left(z_{1}, z_{2}\right)=\mathrm{d}_{\tilde{\mathcal{G}}_{1}}\left(z_{1}\right)$ \\
Cartesian product & $\tilde{\mathcal{G}}_{1} \square \tilde{\mathcal{G}}_{2}$ & $\mathrm{~d}_{\tilde{\mathcal{G}}_{1}} \square \tilde{\mathcal{G}}_{2}\left(z_{1}, z_{2}\right)=\mathrm{d}_{\tilde{\mathcal{G}}_{1}}\left(z_{1}\right)+\mathrm{d}_{\tilde{\mathcal{G}}_{2}}\left(z_{2}\right)$ \\
Semi-strong product & $\tilde{\mathcal{G}}_{1} \bullet \tilde{\mathcal{G}}_{2}$ & $\mathrm{~d}_{\tilde{\mathcal{G}}_{1} \bullet \tilde{\mathcal{G}}_{2}}\left(z_{1}, z_{2}\right)=\mathrm{d}_{\tilde{\mathcal{G}}_{1}}\left(z_{1}\right)+\mathrm{d}_{\tilde{\mathcal{G}}_{2}}\left(z_{2}\right)$ \\
Strong product & $\tilde{\mathcal{G}}_{1} \otimes \tilde{\mathcal{G}}_{2}$ & $\mathrm{~d}_{\tilde{\mathcal{G}}_{1} \otimes \tilde{\mathcal{G}}_{2}}\left(z_{1}, z_{2}\right)=r_{2} \mathrm{~d}_{\tilde{\mathcal{G}}_{1}}\left(z_{1}\right)+\mathrm{d}_{\tilde{\mathcal{G}}_{2}}\left(z_{2}\right)$ \\
Lexicographic product & $\tilde{\mathcal{G}}_{1}\left[\tilde{\mathcal{G}}_{2}\right]$ & $\mathrm{d}_{\tilde{\mathcal{G}}_{1}\left[\tilde{\mathcal{G}}_{2}\right]}\left(z_{1}, z_{2}\right)=r_{2} \mathrm{~d}_{\tilde{\mathcal{G}}_{1}}\left(z_{1}\right)+\mathrm{d}_{\tilde{\mathcal{G}}_{2}}\left(z_{2}\right)$ \\
Symmetric difference & $\tilde{\mathcal{G}}_{1} \oplus \tilde{\mathcal{G}}_{2}$ & $\mathrm{~d}_{\tilde{\mathcal{G}}_{1} \oplus \tilde{\mathcal{G}}_{2}}\left(z_{1}, z_{2}\right)=\left(r_{2}-\mathrm{d}_{G_{2}}\left(z_{2}\right)\right) \mathrm{d}_{\tilde{\mathcal{G}}_{1}}\left(z_{1}\right)+\left(r_{1}-\mathrm{d}_{G_{1}}\left(z_{1}\right)\right) \mathrm{d}_{\tilde{\mathcal{G}}_{2}}\left(z_{2}\right)$ \\
Disjunction & $\tilde{\mathcal{G}}_{1} \vee \tilde{\mathcal{G}}_{2}$ & $\mathrm{~d}_{\tilde{\mathcal{G}}_{1}} \vee \tilde{\mathcal{G}}_{2}\left(z_{1}, z_{2}\right)=r_{2} \mathrm{~d}_{\tilde{\mathcal{G}}_{1}}\left(z_{1}\right)+r_{1} \mathrm{~d}_{\tilde{\mathcal{G}}_{2}}\left(z_{2}\right)-\mathrm{d}_{G_{1}}\left(z_{1}\right) \mathrm{d}_{\tilde{\mathcal{G}}_{2}}\left(z_{2}\right)$ \\
\hline
\end{tabular}

\section{Decision-making approach based on the proposed SIVPFGs}

In decision-making problems, there is a number of uncertainties and in some situations, there exist some relations among criteria in a MCDM problem. So, it is an interesting area for applications of SIVPFG theory. In this section, we develop a multi-criteria decision-making approach based on the above-defined SIVPFSs and SIVPFGs. Further, using a suitable illustration, we demonstrate the developed approach.

\section{Decision-making approach}

Consider a MCDM problem containing a discrete set of $m$ plans (alternatives) $P=\left\{p_{1}, p_{2}, \ldots, p_{m}\right\}$. Let $Z=$ $\left\{z_{1}, z_{2}, \ldots, z_{n}\right\}$ be a set of uncertain agents (criteria), which can be described by a SIVPFS $\left\{\left\langle z,\left(\tilde{\mu}_{\tilde{P}}^{L}(z), \tilde{v}_{\tilde{P}}^{U}(z)\right),\left(\tilde{\mu}_{\tilde{P}}^{U}(z)\right.\right.\right.$, $\left.\left.\left.\tilde{v}_{\tilde{P}}^{L}(z)\right)\right\rangle \mid z \in Z\right\}$, whose weight information is completely unknown. Also each agent was identified with a vertex and links between agents with relations (adges) in SIVPFG. The implementation of any plan will force some or all agents to take actions, during which benefits will be produced. To drive the maximal benefit, how to choose a best plan is a multiagent decision-making problem using SIVPFG. For instance, in the network attacking, actual networks may be confidential, such as internal networks, so a hacker has no idea to how to make this kind of netlike structures clear. In this situation, the hacker can only describe the uncertain network structures by SIVPFGs in accordance with partial reliable information. Thus, the hacker should find a method to solve the MCDM problem using SIVPFG so as to select a plan to damage the network as much as possible.

In a SIVPFG $\tilde{\mathcal{G}}=(\tilde{\mathcal{P}}, \tilde{\mathcal{Q}})$, for a plan, assume that if an agent $z_{i} \in Z$ takes an action, we choose $x_{i}=1$, otherwise $x_{i}=0$. Then the benefit of each agent $z_{i}$ can be calculated using

$$
\begin{aligned}
\tilde{b}_{i}= & \left\langle\left(\tilde{\mu}_{\tilde{P}}^{L}\left(z_{i}\right), \tilde{v}_{\tilde{P}}^{U}\left(z_{i}\right)\right),\left(\tilde{\mu}_{\tilde{P}}^{U}\left(z_{i}\right), \tilde{v}_{\tilde{P}}^{L}\left(z_{i}\right)\right)\right\rangle x_{i} \\
& +\bar{x}_{\mathcal{N}_{i}} \quad i=1,2, \ldots, n .
\end{aligned}
$$

where $\mathcal{N}_{i}$ is the set of the agent $z_{i}$ 's neighbors and

$$
\begin{aligned}
\bar{x}_{\mathcal{N}_{i}}= & \sum_{j \in \mathcal{N}_{i}}\left\langle\left(\tilde{\mu}_{\tilde{Q}^{L}}^{L}\left(z_{i} z_{j}\right), \tilde{v}_{\tilde{Q}}^{U}\left(z_{i} z_{j}\right)\right),\right. \\
& \left.\times\left(\tilde{\mu}_{\tilde{Q}}^{U}\left(z_{i} z_{j}\right), \tilde{v}_{\tilde{Q}}^{L}\left(z_{i} z_{j}\right)\right)\right\rangle \xi\left(z_{i} z_{j}\right) x_{j},
\end{aligned}
$$

$\xi\left(z_{i} z_{j}\right) \in[0,1]$ is the influence coefficient between relevant agents.

Case I: If the weights of all agents are provided, the overall benefit of the plan can be obtained using a simplified intervalvalued Pythagorean fuzzy aggregation operator. Suppose that the simplified interval-valued Pythagorean fuzzy weighted averaging operator is chosen, then the aggregated benefit of the plan can be determined by

$\tilde{b}=\operatorname{SIVPFWA}\left(\tilde{b}_{1}, \tilde{b}_{2}, \ldots, \tilde{b}_{n}\right)=\sum_{i=1}^{n} w_{i} \tilde{b}_{i}, \quad i=1,2, \ldots, n$.

where $w=\left(w_{1}, w_{2}, \ldots, w_{n}\right)^{T}$ is a weight vector of $\left(\tilde{b}_{1}, \tilde{b}_{2}, \ldots, \tilde{b}_{n}\right)^{T}$.

Case II: If the weights of agents are not provided, a method can be developed to calculate the weights of all agents according to some known information, like simplified interval-valued Pythagorean fuzzy graphic structure and the degrees of vertices in a SIVPFG.

$w_{i}=\frac{\mathrm{d}\left(z_{i}\right)}{\sum_{j=1}^{n} \mathrm{~d}\left(z_{j}\right)}, \quad i=1,2, \ldots, n$

Using the above weights and Eq. (2), the overall benefit of a plan can be obtained for the selection of optimal plan.

However, in the SIVPFG-based MCDM problems, if there exist the prioritization relations among the agents, we shall solve this kind of problems by utilizing the prioritized aggregation operators [31] together with the necessary simplified interval-valued Pythagorean fuzzy graphic structure. 

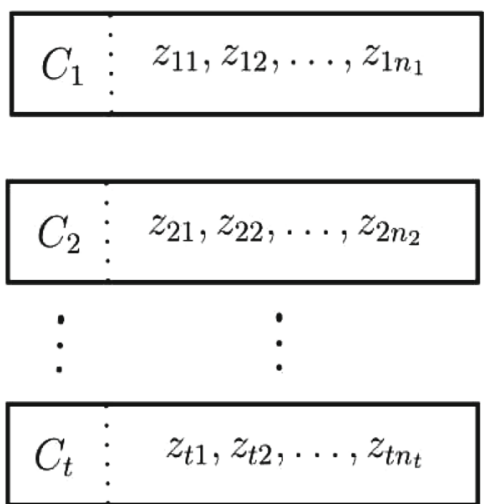

Fig. 9 Prioritized hierarchies of agents (criteria) set

For a SIVPFG, assume that we have a collection of agents (vertices) partitioned into $t$ distinct categories $C_{1}, C_{2}, \ldots, C_{t}$ such that $C_{i}=\left\{z_{i 1}, z_{i 2}, \ldots, z_{i n_{i}}\right\}$, where $z_{i j} \quad(j=$ $\left.1,2, \ldots, n_{i}\right)$ are the agents in the category $C_{i}$ and assume a prioritization relationship among these categories is $C_{1}>$ $C_{2}>\cdots>C_{t}$. The agents in the category $C_{i}$ have a higher priority than those in $C_{j}$ if $i<j$. Then, $Z=\bigcup_{i=1}^{t} C_{i}$ is the universal set of agents and $n=\sum_{i=1}^{t} n_{i}$ is the total number of agents. The prioritized hierarchy structure of $Z$ is given in Fig. 9.

We develop an approach to handle the SIVPFG-based MCDM problems by means of the prioritized simplified interval-valued Pythagorean fuzzy aggregation operators together with the degrees of agents.

Determine the degrees of all agents $d\left(z_{i}\right)(i=1,2, \ldots, n)$ which can be normalized by

$$
\begin{aligned}
\bar{d}\left(z_{i}\right)= & \left\langle\left(\frac{d\left(\tilde{\mu}_{\tilde{P}}^{L}\left(z_{i}\right)\right)}{\sum_{j=1}^{n} d\left(\tilde{\mu}_{\tilde{P}}^{L}\left(z_{j}\right)\right)}, \frac{d\left(\tilde{v}_{\tilde{P}}^{U}\left(z_{i}\right)\right)}{\sum_{j=1}^{n} d\left(\tilde{v}_{\tilde{P}}^{U}\left(z_{j}\right)\right)}\right),\right. \\
& \left.\times\left(\frac{d\left(\tilde{\mu}_{\tilde{P}}^{U}\left(z_{i}\right)\right)}{\sum_{j=1}^{n} d\left(\tilde{\mu}_{\tilde{P}}^{U}\left(z_{j}\right)\right)}, \frac{d\left(\tilde{v}_{\tilde{P}}^{L}\left(z_{i}\right)\right)}{\sum_{j=1}^{n} d\left(\tilde{v}_{\tilde{P}}^{L}\left(z_{j}\right)\right)}\right)\right\rangle, \\
& i=1,2, \ldots, n
\end{aligned}
$$

The weights can be associated with agents dependent upon the satisfaction of the higher priority agent by modeling the prioritization between agents. Then for each category $C_{i}$, we first define

$l_{i}=\left\{\begin{array}{lrl}\langle(1,0),(1,0)\rangle, & i & =0 \\ \varphi\left(\bar{d}\left(z_{i 1}\right), \bar{d}\left(z_{i 2}\right), \ldots, \bar{d}\left(z_{i n_{i}}\right)\right), & i=1,2, \ldots, t\end{array}\right.$

where $\varphi$ is an alternative function such as the minimum or maximum function, the average function, and so on, for calculating $l_{i}$ based on which we determine the weight of each category: $w_{i}=\prod_{k=1}^{i} l_{k-1}, \quad i=1,2, \ldots, t$

Using SIVPFWC operator, the overall benefit of the plan can be calculated as:

$$
\begin{aligned}
\tilde{b}^{\left(p_{i}\right)} & =\operatorname{SIVPFWC}\left(\tilde{b}_{1}, \tilde{b}_{2}, \ldots, \tilde{b}_{n}\right) \\
& =\vee_{i=1}^{t}\left(\vee_{j=1}^{n_{i}}\left(w_{i} \wedge \tilde{b}_{i j}^{(p)}\right)\right)
\end{aligned}
$$

And finally to select a best plan, determine the score functions $s\left(\tilde{b}^{\left(p_{i}\right)}\right)=\frac{1}{2}\left[\left(\left(\tilde{\mu}_{\tilde{\mathcal{P}}}^{L}\right)^{2}-\left(\tilde{v}_{\tilde{\mathcal{P}}}^{U}\right)^{2}\right)+\left(\left(\tilde{\mu}_{\tilde{\mathcal{P}}}^{U}\right)^{2}-\left(\tilde{v}_{\tilde{\mathcal{P}}}^{L}\right)^{2}\right)\right](i=$ $1,2, \ldots, 5)$, and rank all the plans $p_{i}(i=1,2, \ldots, m)$ according to the values of $s\left(\tilde{b}^{\left(p_{i}\right)}\right)(i=1,2, \ldots 5)$.

The processes are modeled as in Fig. 10.

The approach contains the following steps:

Step 1. For a MCDM problem, consider a discrete set of plans (alternatives) $P=\left\{p_{1}, p_{2}, \ldots, p_{m}\right\}$, a set of uncertain agents (criteria) $Z=\left\{z_{1}, z_{2}, \ldots, z_{n}\right\}$ and construction of SIVPFG whose vertices represent the agents considered and edges represent simplified interval-valued Pythagorean fuzzy relations of agents.

Step 2. Determine the degrees of all vertices (agents) in a SIVPFG and normalize them by utilizing Eq. 4 .

Step 3. Designate the prioritization relationships among the agents. Then the collection of agents is partitioned into $t$ distinct categories $C_{1}, C_{2}, \ldots, C_{t}$ such that $C_{i}=\left\{z_{i 1}, z_{i 2}, \ldots, z_{i n_{i}}\right\}$, where $z_{i j}(j=$ $\left.1,2, \ldots, n_{i}\right)$ are the agents in the category $C_{i}$.

Step 4. For each priority category $C_{i}$, determine the values of $l_{i}$ utilizing Eq. 5 .

Step 5. Determine the weight $w_{i}$ of each category by means of $l_{i}, i=1,2, \ldots, t$, using Eq. 6 .

Step 6. Compute the benefit of each agent $z_{i}$ by utilizing Eq. 1.

Step 7. Determine the overall benefit of the plan using SIVPFWC operator from Eq. 7 and select the optimal plan (alternative) according to the score function of the overall benefits of the plans.

\section{Illustrative example}

In this section, the above developed approach has been illustrated with a real-life decision-making problem in simplified interval-valued Pythagorean fuzzy environment.

Consider a discrete set of plans (alternatives) $P=$ $\left\{p_{1}, p_{2}, \ldots, p_{5}\right\}$, a set of uncertain agents (criteria) $Z=$ $\left\{z_{1}, z_{2}, \ldots, z_{7}\right\}$. Under the simplified interval-valued Pythagorean fuzzy environment, we invite the expert to evaluate these alternatives with simplified interval-valued Pythagorean fuzzy elements. Therefore, the 


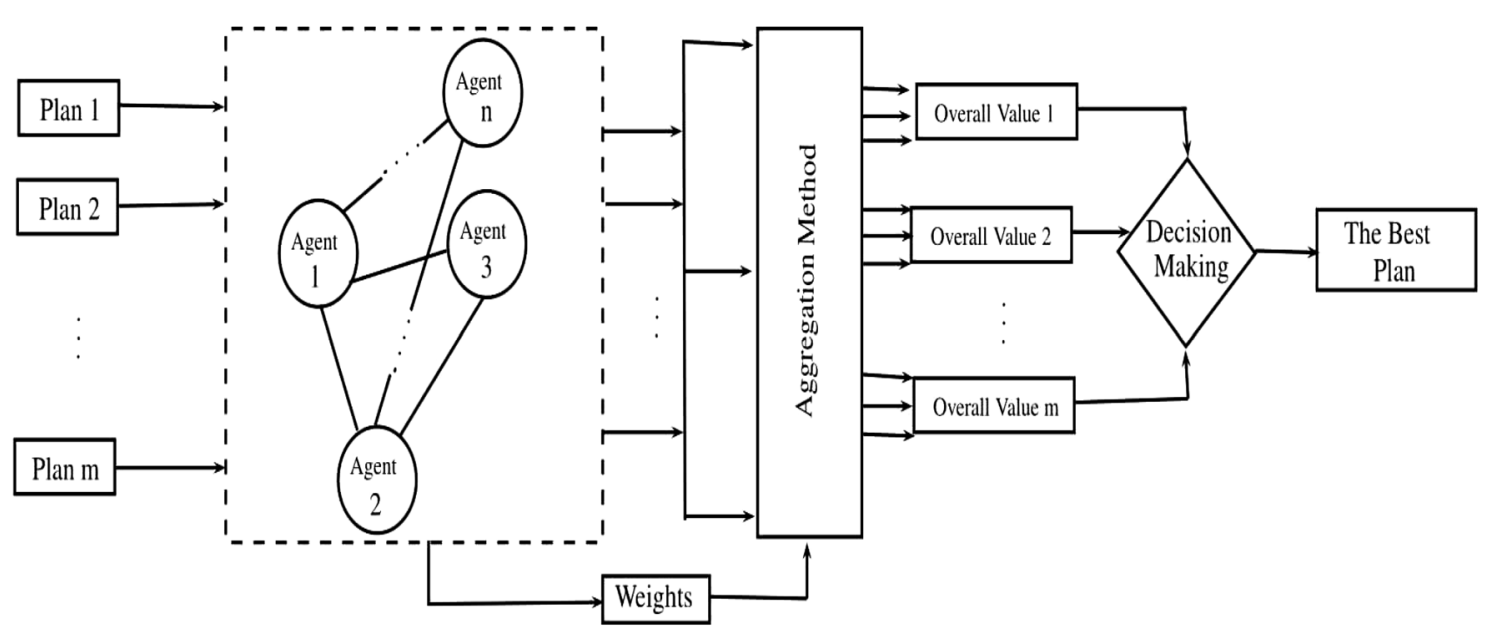

Fig. 10 The MCDM model based on SIVPFG

Table 2 The evaluation information on the plans in simplified interval-valued Pythagorean fuzzy environment

\begin{tabular}{lllll}
\hline & Andrew & John & David & Robert \\
\hline$p_{1}$ & $\langle(0.2,0.6),(0.3,0.4)\rangle$ & $\langle(0.3,0.8),(0.5,0.4)\rangle$ & $\langle(0.5,0.4),(0.7,0.2)\rangle$ & $\langle(0.4,0.3),(0.9,0.2)\rangle$ \\
$p_{2}$ & $\langle(0.3,0.8),(0.6,0.5)\rangle$ & $\langle(0.4,0.5),(0.8,0.3)\rangle$ & $\langle(0.3,0.6),(0.7,0.2)\rangle$ & $\langle(0.2,0.7),(0.5,0.3)\rangle$ \\
$p_{3}$ & $\langle(0.4,0.5),(0.6,0.4)\rangle$ & $\langle(0.2,0.6),(0.7,0.3)\rangle$ & $\langle(0.3,0.5),(0.6,0.4)\rangle$ & $\langle(0.2,0.6),(0.7,0.3)\rangle$ \\
$p_{4}$ & $\langle(0.4,0.7),(0.6,0.4)\rangle$ & $\langle(0.5,0.5),(0.8,0.3)\rangle$ & $\langle(0.2,0.5),(0.6,0.2)\rangle$ & $\langle(0.2,0.4),(0.9,0.3)\rangle$ \\
$p_{5}$ & $\langle(0.1,0.9),(0.3,0.3)\rangle$ & $\langle(0.2,0.8),(0.4,0.5)\rangle$ & $\langle(0.2,0.7),(0.6,0.5)\rangle$ & $\langle(0.3,0.8),(0.6,0.5)\rangle$ \\
\hline & Parker & Smith & & George \\
\hline$p_{1}$ & $\langle(0.5,0.8),(0.6,0.3)\rangle$ & $\langle(0.2,0.4),(0.7,0.3)\rangle$ & $\langle(0.4,0.3),(0.8,0.1)\rangle$ \\
$p_{2}$ & $\langle(0.1,0.8),(0.2,0.3)\rangle$ & $\langle(0.2,0.8),(0.4,0.4)\rangle$ & $\langle(0.4,0.7),(0.5,0.6)\rangle$ \\
$p_{3}$ & $\langle(0.2,0.6),(0.3,0.5)\rangle$ & $\langle(0.2,0.8),(0.6,0.2)\rangle$ & $\langle(0.1,0.7),(0.4,0.3)\rangle$ \\
$p_{4}$ & $\langle(0.2,0.8),(0.3,0.5)\rangle$ & $\langle(0.4,0.5),(0.7,0.1)\rangle$ & $\langle(0.4,0.5),(0.6,0.1)\rangle$ \\
$p_{5}$ & $\langle(0.5,0.6),(0.7,0.3)\rangle$ & $\langle(0.5,0.3),(0.8,0.2)\rangle$ & $\langle(0.5,0.4),(0.7,0.3)\rangle$ \\
\hline
\end{tabular}

simplified interval-valued Pythagorean fuzzy decision matrix is constructed in Table 2. Assume a graph $G=(V, E)$ with seven agents (vertices) $V=\{$ Andrew, John, David, Robert, Parker, Smith, George $\}$ and $E=$ AAndrewJohn, Andrew Robert, GeorgeSmith, GeorgeParker, GeorgeRobert, George David, GeorgeJohn, GeorgeAndrew $\}$. Let $\tilde{\mathcal{G}}=(\tilde{\mathcal{P}}, \tilde{\mathcal{Q}})$ be a SIVPFG of a graph $G$, as shown in Fig. 11 .

Step 1. The degree of each agent is given by

$$
\begin{aligned}
& \mathrm{d}(\text { Andrew })=\langle(0.4,2.2),(0.7,1.5)\rangle, \\
& \mathrm{d}(\text { John })=\langle(0.3,1.7),(0.5,1.1)\rangle, \\
& \mathrm{d}(\text { David })=\langle(0.3,0.7),(0.5,0.4)\rangle, \\
& \mathrm{d}(\text { Robert })=\langle(0.4,1.3),(0.9,0.8)\rangle, \\
& \mathrm{d}(\text { Parker })=\langle(0.1,0.9),(0.3,0.4)\rangle, \\
& \mathrm{d}(\text { Smith })=\langle(0.2,0.9),(0.4,0.6)\rangle, \\
& \mathrm{d}(\text { George })=\langle(1.1,4.7),(2.3,2.8)\rangle .
\end{aligned}
$$

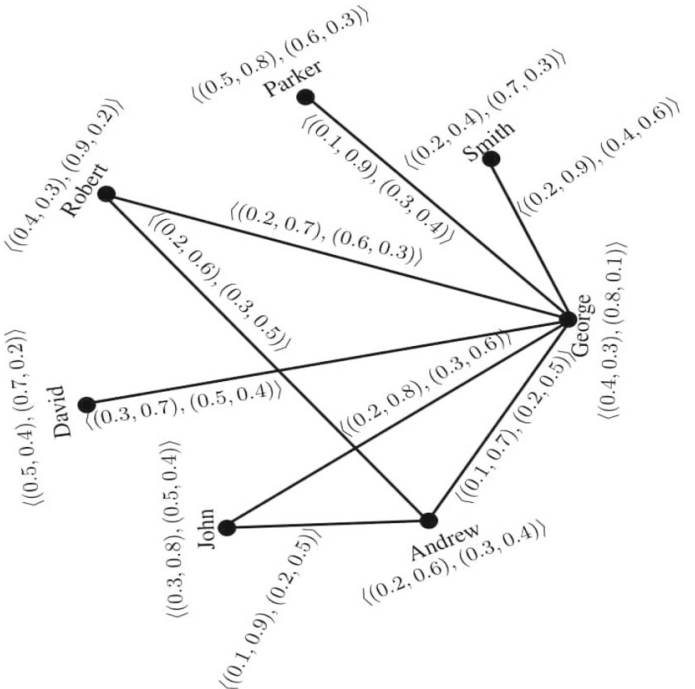

Fig. 11 A SIVPFG with seven vertices (agents) 
The above degrees can be normalized using Eq. (4) as follows:

$\overline{\mathrm{d}}($ Andrew $)=\langle(0.1429,0.1774),(0.1250,0.1974)\rangle$, $\overline{\mathrm{d}}(\mathrm{John})=\langle(0.1071,0.1371),(0.0893,0.1447)\rangle$, $\overline{\mathrm{d}}($ David $)=\langle(0.1071,0.0565),(0.0893,0.0526)\rangle$, $\overline{\mathrm{d}}($ Robert $)=\langle(0.1429,0.1048),(0.1607,0.1053)\rangle$, $\overline{\mathrm{d}}($ Parker $)=\langle(0.0357,0.0726),(0.0536,0.0526)\rangle$, $\overline{\mathrm{d}}($ Smith $)=\langle(0.0714,0.0726),(0.0714,0.0789)\rangle$, $\overline{\mathrm{d}}($ George $)=\langle(0.3929,0.3790),(0.4107,0.3684)\rangle$.

Step 2. Assume that there exist the prioritization simplified interval-valued Pythagorean fuzzy relations $C_{1}=\{$ Andrew, Robert $\}, C_{2}=\{$ David $\}, C_{3}=$ \{John, George $\}, C_{4}=$ (Parker, Smith $\}, C_{i}>C_{j}$ if $i<j(i, j=1,2,3,4)$. So, $n_{1}=2, n_{2}=1, n_{3}=$ $2, n_{4}=2$.

Step 3. Let $\varphi$ be a minimum function, then using Eq. (5), we get

$$
\begin{aligned}
& l_{0}=\langle(1,0),(1,0)\rangle \\
& l_{1}=\langle(0.1429,0.1774),(0.1250,0.1974)\rangle \\
& l_{2}=\langle(0.1071,0.0565),(0.0893,0.0526)\rangle \\
& l_{3}=\langle(0.1071,0.3790),(0.0893,0.3684)\rangle \\
& l_{4}=\langle(0.0357,0.0726),(0.0536,0.0789)\rangle
\end{aligned}
$$

Step 4. Utilizing Eq. (6), the weight of each category can be calculated as:

$$
\begin{aligned}
& w_{1}=l_{0}=\langle(1,0),(1,0)\rangle \\
& w_{2}=l_{0} \otimes l_{1}=\langle(0.1429,0.1774),(0.1250,0.1974)\rangle \\
& w_{3}=l_{0} \otimes l_{1} \otimes l_{2}=\langle(0.0153,0.1859),(0.0112,0.2040)\rangle \\
& w_{4}=l_{0} \otimes l_{1} \otimes l_{2} \otimes l_{3}=\langle(0.0016,0.4162),(0.0010,0.4144)\rangle
\end{aligned}
$$

Step 5. If there is a plan $p_{1}$, in which just agent 'George' takes an action, then $x_{7}=1$ and $x_{i}=0(i=$ $1,2, \ldots, 6)$. Also take $\xi\left(z_{i} z_{j}\right)=0.5$ for $i, j=$ $1,2, \ldots, 7$ and $i \neq j$, then by Eq. (1), the benefits of all agents are:

$$
\begin{aligned}
\tilde{b}_{1}^{(P)}= & \left\langle\left(\tilde{\mu}_{\tilde{\mathcal{P}}}^{L} \text { (Andrew), } \tilde{v}_{\tilde{\mathcal{P}}}^{U}(\text { Andrew })\right),\left(\tilde{\mu}_{\tilde{\mathcal{P}}}^{U}\right. \text { (Andrew), }\right. \\
& \left.\left.\tilde{v}_{\tilde{\mathcal{P}}}^{L}(\text { Andrew })\right)\right\rangle x_{1}+\bar{x}_{\mathcal{N}_{1}} \\
= & \left\langle\left(\tilde{\mu}_{\tilde{\mathcal{Q}}}^{L} \text { (AndrewGeorge }\right), \tilde{v}_{\tilde{\mathcal{Q}}}^{U}(\text { AndrewGeorge })\right), \\
& \left.\left.\left(\tilde{\mu}_{\tilde{\mathcal{Q}}}^{U} \text { (AndrewGeorge }\right), \tilde{v}_{\tilde{\mathcal{Q}}}^{L}(\text { AndrewGeorge })\right)\right\rangle \\
& \xi(\text { AndrewGeorge }) x_{7} \\
= & \langle(0.0708,0.8367),(0.14210 .7071)\rangle, \\
\tilde{b}_{2}^{(P)}= & \left\langle\left(\tilde{\mu}_{\tilde{\mathcal{P}}}^{L}(\text { John }), \tilde{v}_{\tilde{\mathcal{P}}}^{U}(\text { John })\right),\right. \\
& \left.\left(\tilde{\mu}_{\tilde{\mathcal{P}}}^{U}(\text { John }), \tilde{v}_{\tilde{\mathcal{P}}}^{L}(\text { John })\right)\right\rangle x_{2}+\bar{x}_{\mathcal{N}_{2}}
\end{aligned}
$$

$$
\begin{aligned}
& \left.=\left\langle\left(\tilde{\mu}_{\tilde{\mathcal{Q}}}^{L} \text { (JohnGeorge }\right), \tilde{v}_{\tilde{\mathcal{Q}}}^{U} \text { (JohnGeorge }\right)\right), \\
& \left.\left.\left(\tilde{\mu}_{\tilde{\mathcal{Q}}}^{U} \text { (JohnGeorge), } \tilde{v}_{\tilde{\mathcal{Q}}}^{L} \text { (JohnGeorge }\right)\right)\right\rangle \\
& \xi(\text { JohnGeorge }) x_{7} \\
& =\langle(0.1421,0.8944),(0.2146,0.7746)\rangle \text {, } \\
& \tilde{b}_{3}^{(P)}=\left\langle\left(\tilde{\mu}_{\tilde{\mathcal{P}}}^{L}(\text { David }), \tilde{v}_{\tilde{\mathcal{P}}}^{U}(\text { David })\right),\left(\tilde{\mu}_{\tilde{\mathcal{P}}}^{U}\right.\right. \\
& \text { (David), } \left.\tilde{v}_{\tilde{\mathcal{P}}}^{L} \text { (David)) }\right\rangle x_{3}+\bar{x}_{\mathcal{N}_{3}} \\
& =\left\langle\left(\tilde{\mu}_{\tilde{\mathcal{Q}}}^{L} \text { (DavidGeorge) }\right) \tilde{v}_{\tilde{\mathcal{Q}}}^{U}(\text { DavidGeorge })\right), \\
& \left.\left.\left(\tilde{\mu}_{\tilde{\mathcal{Q}}}^{U} \text { (DavidGeorge), } \tilde{v}_{\tilde{\mathcal{Q}}}^{L} \text { (DavidGeorge }\right)\right)\right\rangle \\
& \xi \text { (DavidGeorge) } x_{7} \\
& =\langle(0.2146,0.8367),(0.3660,0.6325)\rangle, \\
& \tilde{b}_{4}^{(P)}=\left\langle\left(\tilde{\mu}_{\tilde{\mathcal{P}}}^{L} \text { (Robert), } \tilde{v}_{\tilde{\mathcal{P}}}^{U} \text { (Robert) }\right),\right. \\
& \left.\left(\tilde{\mu}_{\tilde{\mathcal{P}}}^{U} \text { (Robert), } \tilde{v}_{\tilde{\mathcal{P}}}^{L}(\text { Robert })\right)\right\rangle x_{4}+\bar{x}_{\mathcal{N}_{4}} \\
& =\left\langle\left(\tilde{\mu}_{\tilde{\mathcal{Q}}}^{L} \text { (RobertGeorge), } \tilde{v}_{\tilde{\mathcal{Q}}}^{U}(\text { RobertGeorge })\right),\right. \\
& \text { ( } \left.\left.\tilde{\mu}_{\tilde{\mathcal{Q}}}^{U} \text { (RobertGeorge), } \tilde{v}_{\tilde{\mathcal{Q}}}^{L} \text { (RobertGeorge) }\right)\right\rangle \\
& \xi \text { (RobertGeorge) } x_{7} \\
& =\langle(0.1421,0.8367),(0.4472,0.5477)\rangle \text {, } \\
& \tilde{b}_{5}^{(P)}=\left\langle\left(\tilde{\mu}_{\tilde{\mathcal{P}}}^{L}(\text { Parker }), \tilde{v}_{\tilde{\mathcal{P}}}^{U}(\text { Parker })\right),\right. \\
& \left.\left(\tilde{\mu}_{\tilde{\mathcal{P}}}^{U} \text { (Parker), } \tilde{v}_{\tilde{\mathcal{P}}}^{L}(\text { Parker })\right)\right\rangle x_{5}+\bar{x}_{\mathcal{N}_{5}} \\
& =\left\langle\left(\tilde{\mu}_{\tilde{\mathcal{Q}}}^{L} \text { (ParkerGeorge), } \tilde{v}_{\tilde{\mathcal{Q}}}^{U} \text { (ParkerGeorge) }\right),\right. \\
& \left.\left(\tilde{\mu}_{\tilde{\mathcal{Q}}}^{U} \text { (ParkerGeorge), } \tilde{v}_{\tilde{\mathcal{Q}}}^{L} \text { (ParkerGeorge) }\right)\right\rangle \\
& \xi \text { (ParkerGeorge) } x_{7} \\
& =\langle(0.0708,0.9487),(0.2146,0.6325)\rangle \text {, } \\
& \tilde{b}_{6}^{(P)}=\left\langle\left(\tilde{\mu}_{\tilde{\mathcal{P}}}^{L}(\text { Smith }), \tilde{v}_{\tilde{\mathcal{P}}}^{U}(\text { Smith })\right),\right. \\
& \left.\left(\tilde{\mu}_{\tilde{\mathcal{P}}}^{U}(\text { Smith }), \tilde{v}_{\tilde{\mathcal{P}}}^{L}(\text { Smith })\right)\right\rangle x_{6}+\bar{x}_{\mathcal{N}_{6}} \\
& =\left\langle\left(\tilde{\mu}_{\tilde{\mathcal{Q}}}^{L}(\text { SmithGeorge }), \tilde{v}_{\tilde{\mathcal{Q}}}^{U}(\text { SmithGeorge })\right),\right. \\
& \left.\left(\tilde{\mu}_{\tilde{\mathcal{Q}}}^{U} \text { (SmithGeorge), } \tilde{v}_{\tilde{\mathcal{Q}}}^{L}(\text { SmithGeorge })\right)\right\rangle \\
& \xi \text { (SmithGeorge) } x_{7} \\
& =\langle(0.1421,0.9487),(0.2889,0.7746)\rangle \text {, } \\
& \tilde{b}_{7}^{(P)}=\left\langle\left(\tilde{\mu}_{\tilde{\mathcal{P}}}^{L} \text { (George), } \tilde{v}_{\tilde{\mathcal{P}}}^{U}(\text { George })\right),\right. \\
& \left.\left.\left(\tilde{\mu}_{\tilde{\mathcal{P}}}^{U} \text { (George), } \tilde{v}_{\tilde{\mathcal{P}}}^{L} \text { (George }\right)\right)\right\rangle x_{7}+\bar{x}_{\mathcal{N}_{7}} \\
& =\langle(0.4,0.3),(0.8,0.1)\rangle \text {. }
\end{aligned}
$$

Step 6. By utilizing Eq. (7), we compute the overall benefit of the plane $p_{1}$ as:

$$
\begin{aligned}
\tilde{b}^{\left(p_{1}\right)} & =\vee_{i=1}^{7}\left(\vee_{j=1}^{n_{i}}\left(w_{i} \wedge \tilde{b}_{i j}^{(p)}\right)\right) \\
& =\langle(0.1429,0.3000),(0.4472,0.2040)\rangle \\
s\left(\tilde{b}^{\left(p_{1}\right)}\right) & =0.0444 .
\end{aligned}
$$


Table 3 Comparative analysis

\begin{tabular}{|c|c|c|c|}
\hline Methods & Score of alternatives & & Ranking of alternatives \\
\hline Ashraf et al. [6] & $0.2426 \quad 0.1594 \quad 0.1829$ & $0.2375 \quad 0.1777$ & $p_{1} \succ p_{4} \succ p_{3} \succ p_{5} \succ p_{2}$ \\
\hline Our proposed method & $\begin{array}{lll}0.0444 & 0.0321 & 0.0350\end{array}$ & $0.0430 \quad 0.0311$ & $p_{1} \succ p_{4} \succ p_{3} \succ p_{2} \succ p_{5}$ \\
\hline
\end{tabular}

Analogously, we can determine the score functions of overall benefits of the other plans $p_{i}(i=2,3,4,5)$ :

$$
\begin{aligned}
s\left(\tilde{b}^{\left(p_{2}\right)}\right) & =0.0321,\left(\tilde{b}^{\left(p_{3}\right)}\right)=0.0350, \\
\left(\tilde{b}^{\left(p_{4}\right)}\right) & =0.0430,\left(\tilde{b}^{\left(p_{5}\right)}\right)=0.0311 .
\end{aligned}
$$

Therefore, according to the score function of the overall benefits of the plans $p_{i}(i=1,2,3,4,5)$, these plans can be ranked as:

$p_{1} \succ p_{4} \succ p_{3} \succ p_{2} \succ p_{5}$

\section{Comparative analysis}

Ashraf et al. [6] developed a method for solving the decisionmaking problems based on the permanent function value in the neutrosophic fuzzy environment. We have utilized this approach for the above illustrative example and compared the decision results with the proposed approach of this paper. The results corresponding to these approaches are summarized in Table 3.

From this comparative study, the results obtained by the existing approach coincide with the proposed one which validates the proposed approach. Hence, the proposed approach can be suitably utilized to solve the MCDM problems. Doubtless, several MCDM problems [18,26] with the interrelated criteria have been solved by the existing methods, but these methods can be regarded as the special cases of the graphbased MCDM model.

The novelty of this approach is that a common model of MCDM with the interrelated criteria has been developed and various relationships among the criteria have been described using the corresponding graphical structures in simplified interval-valued Pythagorean fuzzy environment.

\section{Conclusions}

IVPFS constitutes a generalized version of a PFS, in the spirit of interval-valued fuzzy set and accommodates a more complex decision-making situation. However, the representations and calculations of IVPFS seem too complicated. To overcome this drawback, in this research study, we have introduced the new concepts of SIVPFS and corresponding SIVPFN, which is characterized by two PFNs. We have also investigated some aggregation techniques for SIVPFNs.
Further, within the framework of proposed SIVPFS theory, we have developed the novel concept of SIVPFG. SIVPFG, an extended structure of a fuzzy graph, gives more precision, flexibility, and compatibility to the system as compared to the classical, fuzzy and Pythagorean fuzzy models. We have developed a series of operations on SIVPFGs and investigated their properties in detail. We have used the simplified interval-valued Pythagorean fuzzy graphic structure to describe the interrelated criteria in MCDM, and developed the simplified interval-valued Pythagorean fuzzy graph-based multi-agent decision making method. Meanwhile, aiming at lots of the unsettled complex MCDM problems with the interrelated criteria, we can clearly depict the relationships among the criteria and then derive a solution by means of the SIVPFG-based MCDM model. Considering the hesitant information, we are extending our research work to the simplified interval-valued hesitant Pythagorean fuzzy environment and developing its applications.

\section{Compliance with ethical standards}

Conflict of interest The authors declare that they have no conflict of interest regarding the publication of the research article.

Open Access This article is distributed under the terms of the Creative Commons Attribution 4.0 International License (http://creativecomm ons.org/licenses/by/4.0/), which permits unrestricted use, distribution, and reproduction in any medium, provided you give appropriate credit to the original author(s) and the source, provide a link to the Creative Commons license, and indicate if changes were made.

\section{References}

1. Akram M, Dudek WA, Ilyas F (2019) Group decision-making based on pythagorean fuzzy TOPSIS method. Int J Intell Syst. https://doi.org/10.1002/int.22103

2. Akram M, Dar JM, Naz S (2019) Certain graphs under Pythagorean fuzzy environment. Complex Intell Syst. https://doi.org/10.1007/ s40747-018-0089-5

3. Akram M, Habib A, Ilyas F, Dar JM (2018) Specific types of Pythagorean fuzzy graphs and application to decision-making. Math Comput Appl 23(3):42

4. Akram M, Naz S (2018) Energy of Pythagorean fuzzy graphs with applications. Mathematics 6(8):136

5. Akram M, Davvaz B (2012) Strong intuitionistic fuzzy graphs. Filomat 26(1):177-196

6. Ashraf S, Naz S, Rashmanlou H, Malik MA (2017) Regularity of graphs in single valued neutrosophic environment. J Intell Fuzzy Syst 33(1):529-542 
7. Atanassov KT (1986) Intuitionistic fuzzy sets. Fuzzy Sets Syst 20(1):87-96

8. Atanassov K, Gargov G (1989) Interval-valued intuitionistic fuzzy sets. Fuzzy Sets Syst 31(3):343-349

9. Chen TY (2018) An interval-valued pythagorean fuzzy outranking method with a closeness-based assignment model for multiple criteria decision making. Int J Intell Syst 33(1):126-168

10. Enayattabar M, Ebrahimnejad A, Motameni H (2018) Dijkstra algorithm for shortest path problem under interval-valued Pythagorean fuzzy environment. Complex Intell Syst. https://doi. org/10.1007/s40747-018-0083-y

11. Garg H (2019) Hesitant Pythagorean fuzzy Maclaurin symmetric mean operators and its applications to multi attribute decisionmaking process. Int J Intell Syst 34(4):601-626. https://doi.org/ 10.1002/int.22067

12. Garg H (2019) New Logarithmic operational laws and their aggregation operators for Pythagorean fuzzy set and their applications. Int J Intell Syst 34(1):82-106

13. Garg H (2018) Some methods for strategic decision-making problems with immediate probabilities in Pythagorean fuzzy environment. Int J Intell Syst 33(4):687-712

14. Garg H (2018) Linguistic Pythagorean fuzzy sets and its applications in multi-attribute decision-making process. Int J Intell Syst 33(6): 1234-1263

15. Garg H (2016) A novel accuracy function under interval-valued Pythagorean fuzzy environment for solving multicriteria decision making problem. J Intell Fuzzy Syst 31(1):529-540

16. Garg H (2018) A linear programming method based on an improved score function for interval-valued Pythagorean fuzzy numbers and its application to decision-making. Int J Uncertain Fuzzy Knowl Based Syst 26(1):67-80

17. Garg H (2018) New exponential operational laws and their aggregation operators for interval-valued Pythagorean fuzzy multicriteria decision-making. Int J Intell Syst 33(3):653-683

18. Gao H (2018) Pythagorean fuzzy hamacher prioritized aggregation operators in multiple attribute decision making. J Intell Fuzzy Syst 35(2):2229-2245

19. Habib A, Akram M, Farooq A (2019) q-Rung orthopair fuzzy competition graphs with application in the soil ecosystem. Mathematics 7(1):91

20. Mordeson JN, Peng CS (1994) Operations on fuzzy graphs. Inf Sci 79(3):159-170

21. Naz S, Rashmanlou H, Malik MA (2017) Operations on singlevalued neutrosophic graphs with application. J Intell Fuzzy Syst 32(3):2137-2151

22. Naz S, Malik MA, Rashmanlou H (2018) Hypergraphs and transversals of hypergraphs in interval-valued intuitionistic fuzzy setting. J Multiple Value Logic Soft Comput 30(4-6):399-417

23. Naz S, Akram M, Smarandache F (2018) Certain notions of energy in single-valued neutrosophic graphs. Axioms 7(3):50
24. Naz S, Ashraf S, Akram M (2018) A novel approach to decisionmaking with Pythagorean fuzzy information. Mathematics 6(6):95

25. Peng X, Yang Y (2016) Fundamental properties of intervalvalued Pythagorean fuzzy aggregation operators. Int J Intell Syst 31(5):444-487

26. Peng X, Yang Y (2016) Pythagorean fuzzy Choquet integral based MABAC method for multiple attribute group decision making. Int J Intell Syst 31(10):989-1020

27. Ren P, Xu Z, Lei Q (2016) Simplified interval-valued intuitionistic fuzzy sets with intuitionistic fuzzy numbers. J Intell Fuzzy Syst 30(5):2871-2882

28. Rahman K, Abdullah S, Shakeel M, Khan MSA, Ullah M (2017) Interval-valued Pythagorean fuzzy geometric aggregation operators and their application to group decision making problem. Cogent Math Stat 4(1):1-20

29. Rosenfeld A (1975) Fuzzy graphs. In: Zadeh LA, Fu KS, Shimura M (eds) Fuzzy sets and their applications. Academic Press, New York, pp 77-95

30. Wei G, Garg H, Gao H, Wei C (2018) Interval-Valued pythagorean fuzzy Maclaurin symmetric mean operators in multiple-attribute decision making. IEEE Access 6:67866-67884

31. Yager RR (2008) Prioritized aggregation operators. Int J Approx Reason 48:263-274

32. Yager RR (2013) Pythagorean fuzzy subsets. In: Proceedings of Joint IFSA world congress and NAFIPS annual meeting, Edmonton, pp 57-61

33. Yager RR, Abbasov AM (2013) Pythagorean membership grades, complex numbers, and decision making. Int J Intell Syst 28(5):436452

34. Yager RR (2014) Pythagorean membership grades in multi-criteria decision making. IEEE Trans Fuzzy Syst 22(4):958-965

35. Yu X, Xu Z (2012) Graph-based multi-agent decision making. Int J Approx Reason 53(4):502-512

36. Zadeh LA (1975) The concept of a linguistic variable and its application to approximate reasoning-I. Inf Sci 8(3):199-249

37. Zadeh LA (1965) Fuzzy sets. Inf Control 8(3):338-353

Publisher's Note Springer Nature remains neutral with regard to jurisdictional claims in published maps and institutional affiliations. 